\title{
The mean field Schrödinger problem: ergodic behavior, entropy estimates and functional inequalities
}

\author{
Julio Backhoff ${ }^{1,5}$ (D) $\cdot$ Giovanni Conforti $^{2} \cdot$ Ivan $_{\text {Gentil }}{ }^{3} \cdot$ Christian Léonard $^{4}$
}

Received: 27 June 2019 / Revised: 14 May 2020 / Published online: 23 June 2020

(c) The Author(s) 2020

\begin{abstract}
We study the mean field Schrödinger problem (MFSP), that is the problem of finding the most likely evolution of a cloud of interacting Brownian particles conditionally on the observation of their initial and final configuration. Its rigorous formulation is in terms of an optimization problem with marginal constraints whose objective function is the large deviation rate function associated with a system of weakly dependent Brownian particles. We undertake a fine study of the dynamics of its solutions, including quantitative energy dissipation estimates yielding the exponential convergence to equilibrium as the time between observations grows larger and larger, as well as a novel class of functional inequalities involving the mean field entropic cost (i.e. the optimal value in (MFSP)). Our strategy unveils an interesting connection between forward backward stochastic differential equations and the Riemannian calculus on the space of probability measures introduced by Otto, which is of independent interest.
\end{abstract}

Mathematics Subject Classification 93E20 - 60H10 - 65C35 · 91A15 · $39 \mathrm{~B} 72$

$\triangle \quad$ Julio Backhoff

julio.backhoff@univie.ac.at; julio.backhoff@utwente.nl

Giovanni Conforti

giovanni.conforti@polytechnique.edu

Ivan Gentil

gentil@math.univ-lyon1.fr

Christian Léonard

christian.leonard@u-paris10.fr

1 Faculty of Mathematics, University of Vienna, Oskar-Morgenstern-Platz 1, 1090 Vienna, Austria

2 Département de Mathématiques Appliquées, École Polytechnique, Route de Saclay, 91128 Palaiseau Cedex, France

3 Institut Camille Jordan, Univ Claude Bernard Lyon 1, Lyon, France

4 Modal'X, UPL, Univ Paris Nanterre, 92000 Nanterre, France

5 Present Address: Department of Applied Mathematics, University of Twente, Drienerlolaan 5, 7522 NB NBEnschede, The Netherlands 


\section{Contents}

1 Introduction and statement of the main results . . . . . . . . . . . . . . . . . . 476

1.1 Frequently used notation . . . . . . . . . . . . . . . . . . . . . . . . . . 479

1.2 The mean field Schrödinger problem and its equivalent formulations . . . . . . . . . . . . 480

1.2.1 Large deviations principle (LDP) . . . . . . . . . . . . . . . . . . . . . . . . . . . . . . . . 482

1.2.2 McKean-Vlasov control and Benamou-Brenier formulation . . . . . . . . . . . . . 482

1.3 Mean field Schrödinger bridges . . . . . . . . . . . . . . . . . . . . . . . . . . . . 484

1.3.1 Planning McKean-Vlasov FBSDE for MFSB . . . . . . . . . . . . . . . . . . . 485

1.3.2 Schrödinger potentials and the mean field planning PDE system . . . . . . . . . . . . 485

1.4 Convergence to equilibrium and functional inequalities . . . . . . . . . . . . . . . . . . 486

1.4.1 Exponential convergence to equilibrium and the turnpike property . . . . . . . . . . . 487

1.4.2 Functional inequalities for the mean field entropic cost . . . . . . . . . . . . . . . 489

2 Connections with optimal transport . . . . . . . . . . . . . . . . . . . . . . . . . 491

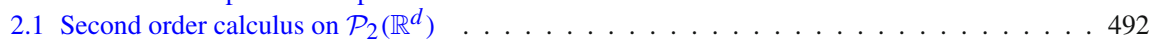

2.2 Newton's laws and FBSDEs . . . . . . . . . . . . . . . . . . . . . . . . . 493

3 The mean field Schrödinger problem and its equivalent formulations: proofs . . . . . . . . . . . 494

3.1 A large deviations principle for particles interacting through their drifts . . . . . . . . . . . 495

3.2 McKean-Vlasov formulation and planning McKean-Vlasov FBSDE . . . . . . . . . . . . 501

3.3 Benamou-Brenier formulation . . . . . . . . . . . . . . . . . . . . 507

3.4 Schrödinger potentials and mean field PDE system: proofs . . . . . . . . . . . . . . . . 509

4 Convergence to equilibrium and functional inequalities: proofs . . . . . . . . . . . . . . . 510

4.1 Exponential upper bound for the corrector . . . . . . . . . . . . . . . . . . 510

4.2 First derivative of $\mathcal{F} \ldots \ldots \ldots \ldots \ldots \ldots \ldots \ldots$

4.3 Time reversal . . . . . . . . . . . . . . . . . . . . . . 515

4.4 Functional inequalities: proofs and the behaviour of $\mathcal{F} \ldots \ldots \ldots$. . . . . . . . . 517

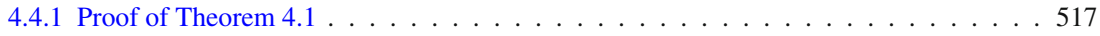

4.4 .2 Proof of Theorem 1.6 . . . . . . . . . . . . . . . . . . . . . . 519

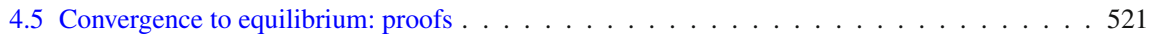

4.5 .1 Proof of Theorem 1.4 . . . . . . . . . . . . . . . . . . 521

4.5 .2 Proof of Theorem $1.5 \ldots \ldots \ldots \ldots 2 \ldots \ldots \ldots$

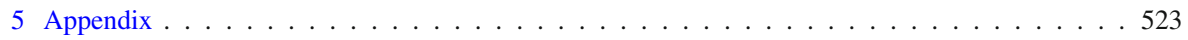

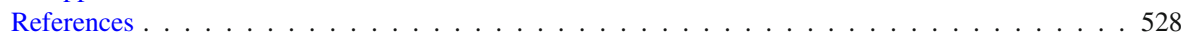

\section{Introduction and statement of the main results}

In the seminal works $[46,47]$ Schrödinger addressed the problem of finding the most likely evolution of a cloud of independent Brownian particles conditionally on the observation of their initial and final configuration. In modern language this is an entropy minimization problem with marginal constraints. The aim of this work is to take the first steps in the understanding of the Mean Field Schrödinger Problem, obtained by replacing in the above description the independent particles by interacting ones.

To obtain an informal description of the problem, consider $N$ Brownian particles $\left(X_{t}^{i, N}\right)_{t \in[0, T], 1 \leq i \leq N}$ interacting through a pair potential $W$

$$
\left\{\begin{aligned}
\mathrm{d} X_{t}^{i, N} & =-\frac{1}{N} \sum_{k=1}^{N} \nabla W\left(X_{t}^{i, N}-X_{t}^{k, N}\right) \mathrm{d} t+\mathrm{d} B_{t}^{i} \\
X_{0}^{i, N} & \sim \mu^{\text {in }} .
\end{aligned}\right.
$$


Their evolution is encoded in the random empirical path measure

$$
\frac{1}{N} \sum_{i=1}^{N} \delta_{X}{ }_{i, N}
$$

At a given time $T$, the configuration of the particle system is visible to an external observer that finds it close to an "unexpected" (écart spontané et considérable in [47]) probability measure $\mu^{\text {fin }}$, namely

$$
\frac{1}{N} \sum_{i=1}^{N} \delta_{X_{T}^{i, N}} \approx \mu^{\mathrm{fin}}
$$

It is a classical result $[4,20,49]$ that the sequence of empirical path measures (2) obeys the large deviations principle (LDP). Thus, the problem of finding the most likely evolution conditionally on the observations is recast as the problem of minimizing the LDP rate function among all path measures whose marginal at time 0 is $\mu^{\text {in }}$ and whose marginal at time $T$ is $\mu^{\text {fin }}$. This is the mean field Schrödinger problem (MFSP). Extending naturally the classical terminology we say that an optimal path measure is a mean field Schrödinger bridge (henceforth MFSB) and the optimal value is the mean field entropic cost. The latter generalizes both the Wasserstein distance and the entropic cost.

The classical Schrödinger problem has been the object of recent intense research activity (see [36]). This is due to the computational advantages deriving from introducing an entropic penalization in the Monge-Kantrovich problem [19] or to its relations with functional inequalities, entropy estimates and the geometrical aspects of optimal transport. Our article contributes to this second line of research, recently explored by the papers $[17,28,32,34,43,44]$. Leaving all precise statements to the main body of the introduction, let us give a concise summary of our contributions.

Dynamics of mean field Schrödinger bridges Our mean field version of the Schrödinger problem stems from fundamental results in large deviations for weakly interacting particle systems such as $[20,49]$ and shares some analogies with the control problems considered in [16] and with the article [2] in which an entropic formulation of second order variational mean field games is studied. Among the more fundamental results we establish for the mean field Schrödinger problem, we highlight

- the existence of MFSBs and, starting from the original large deviations formulation, the derivation of both an equivalent reformulation in terms of a McKean-Vlasov control problem as well as a Benamou-Brenier formula,

- establishing that MFSBs solve forward backward stochastic differential equations (FBSDE) of McKean-Vlasov type (cf. [10,11]).

The proof strategy we adopt in this article combines ideas coming from large deviations and stochastic calculus of variations, see [18,23,52]. Another interesting consequence of having a large deviations viewpoint is that we can also exhibit some regularity 
properties of MFSBs, taking advantage of Föllmer's results [25] on time reversal. Building on $[17,28]$ we establish a link between FBSDEs and the Riemannian calculus on probability measures introduced by Otto [41] that is of independent interest and underlies our proof strategies. In a nutshell, the seminal article [31] established that the heat equation is the gradient flow of the relative entropy w.r.t. the squared Wasserstein distance. Thus, classical first order SDEs yield probabilistic representations for first order ODEs in the Riemannian manifold of optimal transport. Our observation may be seen as the second order counterpart to the results of [31]: indeed we will present an heuristic strongly supporting the fact that Markov solutions of "second order" trajectorial equations (FBSDEs) yield probabilistic representations for second order ODEs in the Riemannian manifold of optimal transport.

Ergodicity of Schrödinger bridges and functional inequalities Consider again (1) and assume that $W$ is convex so that the particle system is rapidly mixing and there is a well defined notion of equilibrium configuration $\mu_{\infty}$. If $N$ and $T$ are large, one expects that

(i) The configurations $\frac{1}{N} \sum_{i=1}^{N} \delta_{X_{t}^{i}}$ at times $t=0, T / 2, T$ are almost independent.

(ii) The configuration at $T / 2$ is with high probability very similar to $\mu_{\infty}$.

Because of (i), even when the external observer acquires the information (3), he/she still expects (ii) to hold. Thus mean field Schrödinger bridges are to spend most of their time around the equilibrium configuration. All our quantitative results originate in an attempt to justify rigorously this claim.

In this work we obtain a number of precise quantitative energy dissipation estimates. These lead us to the main quantitative results of the article:

- we characterize the long time behavior of MFSBs, proving exponential convergence to equilibrium with sharp exponential rates,

- we derive a novel class of functional inequalities involving the mean field entropic cost. Precisely, we obtain a Talagrand inequality and an HWI inequality ${ }^{1}$ that generalize those previously obtained in [12] by Carrillo, McCann and Villani.

Regarding the second point above, we can in fact retrieve (formally) the inequalities in [12] by looking at asymptotic regimes for the mean field Schrödinger problem. Besides the intrinsic interest and their usefulness in establishing some of our main results, our functional inequalities may have consequences in terms of concentration of measure and hypercontractivity of non linear semigroups, but this is left to future work.

The fact that optimal curves of a given optimal control problem spend most of their time around an equilibrium is known in the literature as the turnpike property. The first turnpike theorems have been established in the 60's for problems arising in econometry [39]; general results for deterministic finite dimensional problems are by now available, see [50]. In view of the McKean-Vlasov formulation of the mean field Schrödinger problem, some of our results may be viewed as turnpike theorems as well,

\footnotetext{
1 A Talagrand inequality states that a transportation cost is dominated by a divergence, whereas a HWI inequality states that a divergence is dominated by a transportation cost and a Fisher information.
} 
but for a class of infinite dimensional and stochastic problems. An interesting feature is that, by exploiting the specific structure of our setting, we are able to establish the turnpike property in a quantitative, rather than qualitative form. The McKean-Vlasov formulation also connects our findings with the study of the long time behavior of mean field games [5,7-9].

Concerning the proof methods, our starting point is Otto calculus and the recent rigorous results of [17] together with the heuristics put forward in [28]. The first new ingredient of our proof strategy is the above mentioned connection between FBSDEs and Otto calculus that plays a key role in turning the heuristics into rigorous statements. It is worth remarking that using a trajectorial approach does not just provide with a way of making some heuristics rigorous, but it also permits to obtain a stronger form of some of the results conjectured in [28] which then simply follow by averaging trajectorial estimates. The second new ingredient in our proofs involves a conserved quantity that plays an analogous role to the total energy of a physical system. For such quantity we derive a further functional inequality which seems to be novel already in the classical Schrdinger problem (i.e. for independent particles) and allows to establish the turnpike property.

Structure of the article In the remainder introductory section we state and comment our main results. In Sect. 2 we provide a geometrical interpretation sketching some interesting heuristic connections between optimal transport and stochastic calculus. The material of this section is not used later on; therefore the reader who is not interested in optimal transport may avoid it. Sections 3 and 4 contain the proofs of our main results, the former being devoted to the results concerning the dynamics of MFSBs and the latter one dealing with the ergodic results. Finally an appendix section contains some technical results.

\subsection{Frequently used notation}

- $\left(\Omega, \mathcal{F}_{t}, \mathcal{F}_{T}\right)$ is the canonical space of $\mathbb{R}^{d}$-valued continuous paths on $[0, T]$, so $\left\{\mathcal{F}_{t}\right\}_{t \leq T}$ is the coordinate filtration. $\Omega$ is endowed with the uniform topology.

- $\mathcal{P}(\Omega)$ and $\mathcal{P}\left(\mathbb{R}^{d}\right)$ denote the set of Borel probability measures on $\Omega$ and $\mathbb{R}^{d}$ respectively.

- $\left(X_{t}\right)_{t \in[0, T]}$ is the canonical (i.e. identity) process on $\Omega$.

- $\mathrm{R}^{\mu}$ is the Wiener measure with starting distribution $\mu$.

- $\mathcal{H}(\mathrm{P} \mid \mathrm{Q})$ denotes the relative entropy of $\mathrm{P}$ with respect to $\mathrm{Q}$, defined as $\mathbb{E}_{\mathrm{P}}\left[\log \left(\frac{\mathrm{dP}}{\mathrm{dQ}}\right)\right]$ if $\mathrm{P} \ll \mathrm{Q}$ and $+\infty$ otherwise.

- $\mathrm{P}_{t}$ denotes the marginal distribution of a measure $\mathrm{P} \in \mathcal{P}(\Omega)$ at time $t$.

- $\mathcal{P}_{\beta}(\Omega)$ is the set of measures on $\Omega$ for which $\sup _{t \leq T}\left|X_{t}\right|^{\beta}$ is integrable. $\mathcal{P}_{\beta}\left(\mathbb{R}^{d}\right)$ is the set of measures on $\mathbb{R}^{d}$ for which the function $|\cdot|^{\beta}$ is integrable. 
- The $\beta$-Wasserstein distance on $\mathcal{P}_{\beta}(\Omega)$ is defined by

$$
\mathcal{P}_{\beta}(\Omega)^{2} \ni(\mathrm{P}, \mathrm{Q}) \mapsto \mathcal{W}_{\beta}(\mathrm{P}, \mathrm{Q}):=\left(\inf _{Y \sim \mathrm{P}, Z \sim \mathrm{Q}} \mathbb{E}\left[\sup _{t \in[0, T]}\left|Y_{t}-Z_{t}\right|^{\beta}\right]\right)^{1 / \beta}
$$

With a slight abuse of notation we also denote by $\mathcal{W}_{\beta}$ the $\beta$-Wasserstein distance on $\mathcal{P}_{\beta}\left(\mathbb{R}^{d}\right)$ defined analogously.

- For a given measurable marginal flow $[0, T] \ni t \mapsto \mu_{t} \in \mathcal{P}\left(\mathbb{R}^{d}\right)$, we denote by $L^{2}\left(\left(\mu_{t}\right)_{t \in[0, T]}\right)$ the space of square integrable functions from $[0, T] \times \mathbb{R}^{d}$ to $\mathbb{R}^{d}$ associated to the reference measure $\mu_{t}(\mathrm{~d} x) \mathrm{d} t$ and the corresponding almost-sure identification. We consider likewise the Hilbert space

$$
\mathrm{H}_{-1}\left(\left(\mu_{t}\right)_{t \in[0, T]}\right)
$$

defined as the closure in $L^{2}\left(\left(\mu_{t}\right)_{t \in[0, T]}\right)$ of the smooth subspace

$$
\left\{\Psi:[0, T] \times \mathbb{R}^{d} \rightarrow \mathbb{R}^{d} \text { s.t. } \Psi=\nabla \psi, \psi \in \mathcal{C}_{c}^{\infty}\left([0, T] \times \mathbb{R}^{d}\right)\right\}
$$

- $\gamma$ and $\lambda$ are respectively the standard Gaussian and Lebesgue measure in $\mathbb{R}^{d}$.

- $\mathcal{C}^{l, m}\left([0, T] \times \mathbb{R}^{d} ; \mathbb{R}^{k}\right)$ is the set of functions from $[0, T] \times \mathbb{R}^{d}$ to $\mathbb{R}^{k}$ which have $l$ continuous derivatives in the first (ie. time) variable and $m$ continuous derivatives in the second (ie. space) variable. The space $\mathcal{C}^{m}\left(\mathbb{R}^{d} ; \mathbb{R}^{k}\right)$ is defined in the same way. $\mathcal{C}_{c}^{\infty}\left([0, T] \times \mathbb{R}^{d}\right)$ is the space of real-valued smooth functions on $[0, T] \times \mathbb{R}^{d}$ with compact support. The gradient $\nabla$ and Laplacian $\Delta$ act only in the space variable.

- If $f$ is a function and $\mu$ a measure, its convolution is $x \mapsto f * \mu(x):=\int f(x-$ y) $\mu(\mathrm{d} y)$.

\subsection{The mean field Schrödinger problem and its equivalent formulations}

We are given a so-called interaction potential $W: \mathbb{R}^{d} \rightarrow \mathbb{R}$, for which we assume

$$
\begin{aligned}
& W \text { is of class } \mathcal{C}^{2}\left(\mathbb{R}^{d} ; \mathbb{R}\right) \text { and symmetric, i.e. } W(\cdot)=W(-\cdot), \\
& \qquad \sup _{z, v \in \mathbb{R}^{d},|v|=1} v \cdot \nabla^{2} W(z) \cdot v<+\infty .
\end{aligned}
$$

Besides the interaction potential, the data of the problem are a pair of probability measures $\mu^{\text {in }}, \mu^{\text {fin }}$ on which we impose

$$
\mu^{\text {in }}, \mu^{\text {fin }} \in \mathcal{P}_{2}\left(\mathbb{R}^{d}\right) \text { and } \tilde{\mathcal{F}}\left(\mu^{\text {in }}\right), \tilde{\mathcal{F}}\left(\mu^{\text {fin }}\right)<+\infty,
$$


where the free energy or entropy functional $\tilde{\mathcal{F}}$ is defined for $\mu \in \mathcal{P}_{2}\left(\mathbb{R}^{d}\right)$ by

$$
\tilde{\mathcal{F}}(\mu)= \begin{cases}\int_{\mathbb{R}^{d}} \log \mu(x) \mu(\mathrm{d} x)+\int_{\mathbb{R}^{d}} W * \mu(x) \mu(\mathrm{d} x), & \text { if } \mu \ll \lambda \\ +\infty, & \text { otherwise. }\end{cases}
$$

In the above, and in the rest of the article, we shall make no distinction between a measure and its density against Lebesgue measure $\lambda$, provided it exists.

We recall that the McKean-Vlasov dynamics is the non linear SDE

$$
\left\{\begin{array}{l}
\mathrm{d} Y_{t}=-\nabla W * \mu_{t}\left(Y_{t}\right) \mathrm{d} t+\mathrm{d} B_{t} \\
Y_{0} \sim \mu^{\mathrm{in}}, \quad \mu_{t}=\operatorname{Law}\left(Y_{t}\right), \quad \forall t \in[0, T]
\end{array}\right.
$$

Under the hypothesis (H1), it is a classical result (see e.g. [13, Thm 2.6]) that (5) admits a unique strong solution whose law we denote $\mathrm{P}^{\mathrm{MKV}}$. The functional $\tilde{\mathcal{F}}$ plays a crucial role in the sequel. For the moment, let us just remark that the marginal flow of the McKean-Vlasov dynamics may be viewed as the gradient flow of $\frac{1}{2} \tilde{\mathcal{F}}$ in the Wasserstein space $\left(\mathcal{P}_{2}\left(\mathbb{R}^{d}\right), \mathcal{W}_{2}(\cdot, \cdot)\right)$.

If $\mathrm{P} \in \mathcal{P}_{1}(\Omega)$ is given, then the stochastic differential equation

$$
\left\{\begin{aligned}
\mathrm{d} Z_{t} & =-\nabla W * \mathrm{P}_{t}\left(Z_{t}\right) \mathrm{d} t+\mathrm{d} B_{t} \\
Z_{0} & \sim \mu^{\mathrm{in}}
\end{aligned}\right.
$$

admits a unique strong solution (cf. Sect. 3.2) whose law we denote $\Gamma(\mathrm{P})$. With this we can now introduce the main object of study of the article:

Definition 1.1 The mean field Schrödinger problem ${ }^{2}$ is

$$
\inf \left\{\mathcal{H}(\mathrm{P} \mid \Gamma(\mathrm{P})): \mathrm{P} \in \mathcal{P}_{1}(\Omega), \mathrm{P}_{0}=\mu^{\mathrm{in}}, \mathrm{P}_{T}=\mu^{\mathrm{fin}}\right\}
$$

Its optimal value, denoted $\mathscr{C}_{T}\left(\mu^{\mathrm{in}}, \mu^{\mathrm{fin}}\right)$, is called mean field entropic transportation cost. Its optimizers are called mean field Schrödinger bridges (MFSB).

It is not difficult to provide existence of optimizers for (MFSP). In the classical case, uniqueness is an easy consequence of the convexity of the entropy functional. However, the rate function $\mathcal{H}(\mathrm{P} \mid \Gamma(\mathrm{P}))$ is not convex in general.

Proposition 1.1 Grant (H1), (H2). Then (MFSP) admits at least an optimal solution.

Remark 1.1 The dynamics of the McKean-Vlasov dynamics for the particle system (1) displays a wide array of different behaviors, including phase transitions, see [51] for example. Thus, we do not expect uniqueness of mean field Schödinger bridges in

\footnotetext{
2 The choice of $W$ as interaction mechanism is a particular one. Thus (MFSP) is not the only mean field Schrödinger problem of interest. It would have been easy to include in the dynamics a confinement (singlesite) potential. However, since one of the goals of this article is to understand the role of the pair potential $W$, we preferred not to do that, as the single site potential may be the one that determines the long time behavior of mean field Schrödinger bridges.
} 
general. However, in the case when $W$ is convex, although the rate function $\mathcal{H}(\mathrm{P} \mid \Gamma(\mathrm{P}))$ is not convex in the usual sense, the entropy $\mathcal{F}$ is displacement convex in the sense of McCann [38]. This observation was indeed used to prove uniqueness of minimizers for $\mathcal{F}$, and could be the starting point towards uniqueness for (MFSP).

\subsubsection{Large deviations principle (LDP)}

We start by deriving the LDP interpretation of (MFSP). Recall the interacting particle system $\left(X_{t}^{i, N}\right)_{t \in[0, T], 1 \leq i \leq N}$ of (1). The theory of stochastic differential equations guarantees the strong existence and uniqueness for this particle system under (H1), (H2). In the next theorem we obtain a LDP for the sequence of empirical path measures; in view of the classical results of [20], it is not surprising that the LDP holds. However, even the most recent works on large deviations for weakly interacting particle systems such as [4] do not seem to cover the setting and scope of Theorem 1.1. Essentially, this is because in those references the LDPs are obtained for a topology that is weaker than the $\mathcal{W}_{1}$-topology, that is what we need later on.

Theorem 1.1 In addition to $(\mathrm{H} 1),(\mathrm{H} 2)$ assume that

$$
\int_{\mathbb{R}^{d}} \exp (r|x|) \mu^{\text {in }}(\mathrm{d} x)<\infty \text { for all } r>0 .
$$

Then the sequence of empirical measures

$$
\left\{\frac{1}{N} \sum_{i=1}^{N} \delta_{X^{i, N}} ; N \in \mathbb{N}\right\}
$$

satisfies the LDP on $\mathcal{P}_{1}(\Omega)$ equipped with the $\mathcal{W}_{1}$-topology, with good rate function given by

$$
\mathcal{P}_{1}(\Omega) \ni \mathrm{P} \mapsto \mathscr{I}(\mathrm{P}):= \begin{cases}\mathcal{H}(\mathrm{P} \mid \Gamma(\mathrm{P})), & \mathrm{P} \ll \Gamma(\mathrm{P}), \\ +\infty, & \text { otherwise }\end{cases}
$$

In fact we will prove in Sect. 3 a strengthened version of Theorem 1.1 where the drift term is much more general. For this, we will follow Tanaka's elegant reasoning [49].

Remark 1.2 Having a rate function implies $\operatorname{Prob}\left[\frac{1}{N} \sum_{i=1}^{N} \delta_{X^{i}, N} \approx \mathrm{P}\right] \approx \exp (-N \mathscr{I}(\mathrm{P}))$ heuristically. Hence Problem (MFSP) has the desired interpretation of finding the most likely evolution of the particle system conditionally on the observations (when $N$ is very large).

\subsubsection{McKean-Vlasov control and Benamou-Brenier formulation}

We now reinterpret the mean field Schrödinger problem (MFSP) in terms of McKeanVlasov stochastic control (also known as mean field control). 
Lemma 1.1 Let $\mathrm{P}$ be admissible for (MFSP). There exists a predictable process $\left(\alpha_{t}^{\mathrm{P}}\right)_{t \in[0, T]}$ s.t.

$$
\mathbb{E}_{\mathrm{P}}\left[\int_{0}^{T}\left|\alpha_{t}^{\mathrm{P}}\right|^{2} \mathrm{~d} t\right]<+\infty
$$

and so that

$$
X_{t}-\int_{0}^{t}\left(-\nabla W * \mathrm{P}_{s}\left(X_{s}\right)+\alpha_{s}^{\mathrm{P}}\right) \mathrm{d} s
$$

has law $R^{\mu^{\text {in }}}$ under P. The problem (MFSP) is equivalent to

$$
\inf \left\{\frac{1}{2} \mathbb{E}_{\mathrm{P}}\left[\int_{0}^{T}\left|\alpha_{t}^{\mathrm{P}}\right|^{2} \mathrm{~d} t\right]: \mathrm{P} \in \mathcal{P}_{1}(\Omega), \mathrm{P}_{0}=\mu^{\mathrm{in}}, \mathrm{P}_{T}=\mu^{\mathrm{fin}}, \alpha^{\mathrm{P}} \text { as in (9) }\right\},
$$

as well as to

$$
\begin{aligned}
& \inf \frac{1}{2} \mathbb{E}_{\mathrm{P}}\left[\int_{0}^{T}\left|\Phi_{t}+\nabla W * \mathrm{P}_{t}\left(X_{t}\right)\right|^{2} \mathrm{~d} t\right] \\
& \text { s.t. } \mathrm{P} \in \mathcal{P}_{1}(\Omega), \mathrm{P}_{0}=\mu^{\text {in }}, \mathrm{P}_{T}=\mu^{\mathrm{fin}}, \mathrm{P} \circ\left(X .-\int_{0}^{.} \Phi_{s} \mathrm{~d} s\right)^{-1}=R^{\mu^{\text {in }}} .
\end{aligned}
$$

The formulations (10)-(11) can be seen as McKean-Vlasov stochastic control problems. In the first case one is steering through $\alpha^{\mathrm{P}}$ part of the drift of a McKean-Vlasov SDE. In the second case one is controlling the drift $\Phi$ of a standard SDE but the optimization cost depends non-linearly on the law of the controlled process. In both cases, the condition $\mathrm{P}_{T}=\mu^{\text {fin }}$ is rather unconventional. By analogy with the theory of mean field games, one could refer to (10)-(11) as planning McKean-Vlasov stochastic control problems, owing to this type of terminal condition.

The third and last formulation of (MFSP) we propose relates to the well known fluid dynamics representation of the Monge Kantorovich distance due to Benamou and Brenier (cf. [53]) that has been recently extended to the standard entropic transportation cost $[15,27]$. The interest of this formula is twofold: on the one hand it clearly shows that (MFSP) is equivalent and gives a rigorous meaning to some of the generalized Schrödinger problems formally introduced in [28,34]. On the other hand, it allows to interpret (MFSP) as a control problem in the Riemannian manifold of optimal transport. This viewpoint, that we shall explore in more detail in Sect. 2, provides with a strong guideline towards the study of the long time behavior of Schrödinger bridges.

We define the set $\mathcal{A}$ as the collection of all absolutely continuous curves $\left(\mu_{t}\right)_{t \in[0, T]} \subset \mathcal{P}_{2}\left(\mathbb{R}^{d}\right)$ (cf. Sect. 4.2) such that $\mu_{0}=\mu^{\text {in }}, \mu_{T}=\mu^{\text {fin }}$ and

$$
\begin{aligned}
(t, z) & \mapsto \nabla \log \mu_{t}(z) \in L^{2}\left(\mathrm{~d} \mu_{t} \mathrm{~d} t\right), \\
(t, z) & \mapsto \nabla W * \mu_{t}(z) \in L^{2}\left(\mathrm{~d} \mu_{t} \mathrm{~d} t\right) .
\end{aligned}
$$


We then define

$$
\begin{aligned}
\mathscr{C}_{T}^{B B}\left(\mu^{\mathrm{in}}, \mu^{\mathrm{fin}}\right):= & \inf _{\substack{\left(\mu_{t}\right)_{t \in[0, T] \in \mathcal{A}}, \partial_{t} \mu_{t}+\nabla \cdot\left(w_{t} \mu_{t}\right)=0}} \frac{1}{2} \int_{0}^{T} \int_{\mathbb{R}^{d}} \mid w_{t}(z) \\
& +\frac{1}{2} \nabla \log \mu_{t}(z)+\left.\nabla W * \mu_{t}(z) s\right|^{2} \mu_{t}(\mathrm{~d} z) \mathrm{d} t .
\end{aligned}
$$

Theorem 1.2 Let (H1), (H2) hold. Then

$$
\mathscr{C}_{T}\left(\mu^{\mathrm{in}}, \mu^{\mathrm{fin}}\right)=\mathscr{C}_{T}^{B B}\left(\mu^{\mathrm{in}}, \mu^{\mathrm{fin}}\right) .
$$

If $\mathrm{P}$ is optimal for (MFSP) and the latter is finite, then $\left(\mathrm{P}_{t}\right)_{t \in[0, T]}$ is optimal in (12) and its associated tangent vector field $w$ is given by

$$
-\nabla W * \mathrm{P}_{t}(z)+\Psi_{t}(z)-\frac{1}{2} \nabla \log \mathrm{P}_{t}
$$

where $\Psi$ is as in Theorem 1.3 below.

Conversely, if $\left(\mu_{t}\right)_{t \in[0, T]}$ is optimal for $\mathscr{C}_{T}^{B B}\left(\mu^{\mathrm{in}}, \mu^{\mathrm{fin}}\right)$ and the latter is finite, then there exists an optimizer of $\mathscr{C}_{T}\left(\mu^{\mathrm{in}}, \mu^{\mathrm{fin}}\right)$ whose marginal flow equals $\left(\mu_{t}\right)_{t \in[0, T]}$.

\subsection{Mean field Schrödinger bridges}

Leveraging the stochastic control interpretation, and building on the stochastic calculus of variations perspective, we obtain the following necessary optimality conditions for (MFSP).

Theorem 1.3 Assume (H1), (H2) and let $\mathrm{P}$ be optimal for (MFSP). Then there exist $\Psi \in \mathrm{H}_{-1}\left(\left(\mathrm{P}_{t}\right)_{t \in[0, T]}\right)$ such that

$$
(\mathrm{d} t \times \mathrm{dP} \text {-a.s. }) \quad \alpha_{t}^{\mathrm{P}}=\Psi_{t}\left(X_{t}\right),
$$

where $\left(\alpha_{t}^{\mathrm{P}}\right)_{t \in[0, T]}$ is related to $\mathrm{P}$ as in Lemma 1.1. The process $t \mapsto \Psi_{t}\left(X_{t}\right)$ is continuous $^{3}$ and the process $\left(M_{t}\right)_{t \in[0, T]}$ defined by

$$
M_{t}:=\Psi_{t}\left(X_{t}\right)-\int_{0}^{t} \tilde{\mathbb{E}}_{\tilde{\mathrm{P}}}\left[\nabla^{2} W\left(X_{s}-\tilde{X}_{s}\right) \cdot\left(\Psi_{s}\left(X_{s}\right)-\Psi_{s}\left(\tilde{X}_{s}\right)\right)\right] \mathrm{d} s
$$

is a continuous martingale under $\mathrm{P}$ on $\left[0, T\left[\right.\right.$, where $\left(\tilde{X}_{t}\right)_{t \in[0, T]}$ is an independent copy of $\left(X_{t}\right)_{t \in[0, T]}$ defined on some probability space $(\tilde{\Omega}, \tilde{\mathfrak{F}}, \tilde{\mathrm{P}})$ and $\tilde{\mathbb{E}}_{\tilde{\mathrm{P}}}$ denotes the expectation on $(\tilde{\Omega}, \tilde{\mathfrak{F}}, \tilde{\mathrm{P}})$.

We shall refer to $\Psi$ as the corrector of P. Correctors will play an important role in the ergodic results. In this part, we give an interpretation of Theorem 1.1 in terms of stochastic analysis (FBSDEs) and partial differential equations.

\footnotetext{
${ }^{3}$ More precisely, it has a continuous version adapted to the P-augmented canonical filtration. 


\subsubsection{Planning McKean-Vlasov FBSDE for MFSB}

We consider the following McKean Vlasov forward-backward stochastic differential equation (FBSDE) in the unknowns $(X, Y, Z)$ :

$$
\left\{\begin{array}{l}
\mathrm{d} X_{t}=-\tilde{\mathbb{E}}\left[\nabla W\left(X_{t}-\tilde{X}_{t}\right)\right] \mathrm{d} t+Y_{t} \mathrm{~d} t+\mathrm{d} B_{t} \\
\mathrm{~d} Y_{t}=\tilde{\mathbb{E}}\left[\nabla^{2} W\left(X_{t}-\tilde{X}_{t}\right) \cdot\left(Y_{t}-\tilde{Y}_{t}\right)\right] \mathrm{d} t+Z_{t} \cdot \mathrm{d} B_{t} \\
X_{0} \sim \mu^{\mathrm{in}}, X_{T} \sim \mu^{\mathrm{fin}} .
\end{array}\right.
$$

As in the stochastic control interpretation of the mean field Schrödinger problem, here too the terminal condition $X_{T} \sim \mu^{\text {fin }}$ is somewhat unconventional. We hence call this forward-backward system the planning McKean-Vlasov FBSDE.

Thanks to the results in Sect. 1.2.2 we can actually solve (15). If P is optimal for (MFSP) with associated $\Psi$ as recalled in Theorem 1.3 above, all we need to do is take $Y_{t}:=\Psi_{t}\left(X_{t}\right)$ and reinterpret (9) for the dynamics of the canonical process $X$ and (14) for the dynamics of $Y$ (in the latter case using martingale representation).

One remarkable aspect of this connection between Schrödinger problems and FBSDEs is that one can prove existence of solutions to such FBSDEs by a purely variational method. Indeed, we remark that (15) is beyond the scope of existing FBSDE theory, such as Carmona and Delarue's [11, Theorem 5.1]. Further, we also obtained for free an extra bit of information: the constructed process $Y$ lives in $\mathrm{H}_{-1}\left(\left(\mathrm{P}_{t}\right)_{t \in[0, T]}\right)$. This is in tandem with the usual heuristic relating FBSDEs and PDEs (where $Y$ is conjectured to be an actual gradient) as explained in Carmona and Delarue's [10, Remark 3.1]. In fact, if we make the additional assumption that $Y_{t}=\nabla \psi_{t}\left(X_{t}\right)$ for some potential $\psi_{t}(x)$, and we set $\mu_{t}=\left(X_{t}\right)_{\#} \mathrm{P}$, then after some computations we arrive at the PDE system ${ }^{4}$ :

$$
\left\{\begin{array}{l}
\partial_{t} \mu_{t}(x)-\frac{1}{2} \Delta \mu_{t}(x)+\nabla \cdot\left(\left(-\nabla W * \mu_{t}(x)+\nabla \psi_{t}(x)\right) \mu_{t}(x)\right)=0 \\
\partial_{t} \nabla \psi_{t}(x)+\frac{1}{2} \nabla \Delta \psi_{t}(x)+\nabla^{2} \psi_{t}(x) \cdot\left(-\nabla W * \mu_{t}(x)+\nabla \psi_{t}(x)\right) \\
=\int_{\mathbb{R}^{d}} \nabla^{2} W(x-\tilde{x}) \cdot\left(\nabla \psi_{t}(x)-\nabla \psi_{t}(\tilde{x})\right) \mu_{t}(\mathrm{~d} \tilde{x}), \\
\mu_{0}(x)=\mu^{\mathrm{in}}(x), \mu_{T}(x)=\mu^{\mathrm{fin}}(x) .
\end{array}\right.
$$

\subsubsection{Schrödinger potentials and the mean field planning PDE system}

The PDE system (16) is the literal translation of the planning McKean-Vlasov FBSDE in the case when the process $Y$ is an actual gradient, $Y=\nabla \psi$. In the next corollary we show that if this is the case, and if $\psi$ is sufficiently regular, then (16) can be rewritten as a system of two coupled PDEs, the first being a Hamilton-Jacobi-Bellman equation for $\psi$, and the second one being a Fokker-Planck equation. This type of PDE system is the prototype of a planning mean field game [33].

\footnotetext{
4 The Laplacian of a vectorial function is defined coordinate-wise.
} 
Corollary 1.1 Let $\mathrm{P}$ be an optimizer for (MFSP), $\Psi .(\cdot)$ be as in Theorem 1.3 and set $\mu_{t}=\mathrm{P}_{t}$ for all $t \in[0, T]$. If $\mu .(\cdot)$ is everywhere positive and of class $\mathcal{C}^{1,2}([0, T] \times$ $\left.\mathbb{R}^{d} ; \mathbb{R}\right)$ and $\Psi .(\cdot)$ is of class $\mathcal{C}^{1,2}\left([0, T] \times \mathbb{R}^{d} ; \mathbb{R}^{d}\right)$ then there exists $\psi:[0, T] \times \mathbb{R}^{d} \rightarrow$ $\mathbb{R}$ such that $\Psi_{t}(x)=\nabla \psi_{t}(x)$ for all $(t, x) \in[0, T] \times \mathbb{R}^{d}$. Moreover, $(\psi .(\cdot), \mu .(\cdot))$ form a classical solution of

$$
\left\{\begin{array}{l}
\partial_{t} \psi_{t}(x)+\frac{1}{2} \Delta \psi_{t}(x)+\frac{1}{2}\left|\nabla \psi_{t}(x)\right|^{2}=\int_{\mathbb{R}^{d}} \nabla W(x-\tilde{x}) \cdot\left(\nabla \psi_{t}(x)-\nabla \psi_{t}(\tilde{x})\right) \mu_{t}(\mathrm{~d} \tilde{x}), \\
\partial_{t} \mu_{t}(x)-\frac{1}{2} \Delta \mu_{t}(x)+\nabla \cdot\left(\left(-\nabla W * \mu_{t}(x)+\nabla \psi_{t}(x)\right) \mu_{t}(x)\right)=0 \\
\mu_{0}(x)=\mu^{\mathrm{in}}(x), \mu_{T}(x)=\mu^{\mathrm{fin}}(x)
\end{array}\right.
$$

A fundamental result [26,57] concerning the structure of optimizers in the classical Schrödinger problem is that their density takes a product form, i.e.

$$
\mu_{t}=\exp \left(\psi_{t}+\varphi_{t}\right)
$$

where $\varphi_{t}(x), \psi_{t}(x)$ solve respectively the forward and backward Hamilton Jacobi Bellman equation

$$
\left\{\begin{aligned}
\partial_{t} \psi+\frac{1}{2} \Delta \psi+\frac{1}{2}|\nabla \psi|^{2} & =0 \\
-\partial_{t} \varphi+\frac{1}{2} \Delta \varphi+\frac{1}{2}|\nabla \varphi|^{2} & =0
\end{aligned}\right.
$$

It is interesting to see that this structure is preserved in (MFSP), at least formally. The effect of having considered interacting Brownian particles instead of independent ones is reflected in the fact that the two Hamilton-Jacobi-Bellman PDEs are coupled not only through the boundary conditions but also through their dynamics.

Corollary 1.2 Using the same notation and under the same hypotheses of Corollary 1.1, if we define $\varphi:[0, T] \times \mathbb{R}^{d} \rightarrow \mathbb{R}$ via

$$
\mu_{t}=\exp \left(-2 W * \mu_{t}+\varphi_{t}+\psi_{t}\right)
$$

then $(\psi \cdot(\cdot), \varphi .(\cdot))$ solves

$$
\left\{\begin{aligned}
\partial_{t} \psi_{t}(x)+\frac{1}{2} \Delta \psi_{t}(x)+\frac{1}{2}\left|\nabla \psi_{t}(x)\right|^{2}=\int \nabla W(x-\tilde{x}) \cdot\left(\nabla \psi_{t}(x)-\nabla \psi_{t}(\tilde{x})\right) \mu_{t}(\mathrm{~d} \tilde{x}), \\
-\partial_{t} \varphi_{t}(x)+\frac{1}{2} \Delta \varphi_{t}(x)+\frac{1}{2}\left|\nabla \varphi_{t}(x)\right|^{2}=\int \nabla W(x-\tilde{x}) \cdot\left(\nabla \varphi_{t}(x)-\nabla \varphi_{t}(\tilde{x})\right) \mu_{t}(\mathrm{~d} \tilde{x}) .
\end{aligned}\right.
$$

\subsection{Convergence to equilibrium and functional inequalities}

Our aim is to show that MFSBs spend most of their time in a small neighborhood of the equilibrium configuration $\mu_{\infty}$, to study their long time behavior, and to derive a new class of functional inequalities involving the mean field entropic $\operatorname{cost} \mathscr{C}_{T}\left(\mu^{\mathrm{in}}, \mu^{\mathrm{fin}}\right)$. 
Throughout this section we make the assumption that $W$ is uniformly convex, ie. that

$$
\exists \kappa>0 \text { s.t. } \forall z \in \mathbb{R}^{d}, \quad \nabla^{2} W(z) \geq \kappa \mathbb{I}_{d \times d},
$$

where the inequality above has to be understood as an inequality between quadratic forms. Under (H3) the McKean Vlasov dynamics associated with the particle system (1) converges in the limit as $T \rightarrow+\infty$ to an equilibrium measure $\mu_{\infty}$, that is found by minimizing the functional $\tilde{\mathcal{F}}$ over the elements of $\mathcal{P}_{2}\left(\mathbb{R}^{d}\right)$ whose mean is the same as $\mu^{\text {in }}$. Existence and uniqueness of $\mu_{\infty}$ has been proven in [38].

We shall often assume that $\mu^{\text {in }}$ and $\mu^{\text {fin }}$ have the same mean:

$$
\int_{\mathbb{R}^{d}} x \mu^{\mathrm{in}}(\mathrm{d} x)=\int_{\mathbb{R}^{d}} x \mu^{\mathrm{fin}}(\mathrm{d} x) .
$$

Remark 1.3 Assumption (H3) is a classical one ensuring exponential convergence rates for the McKean-Vlasov dynamics. It may be weakened in various ways, see the work [12] by Carrillo, McCann and Villani or the more recent [3] by Bolley, Gentil and Guillin, for instance. It is an interesting question to determine which of the results of this section still hold in the more general setup. Hypothesis (H4) can be easily removed using the fact that the mean evolves linearly along any Schrödinger bridge (see Lemma 4.2 below). We insist that the only key assumption is (H3).

Long time behavior of mean field games The articles [5,7-9] study the asymptotic behaviour of dynamic mean field games showing convergence towards an ergodic mean field game with exponential rates. Following [33], we can associate to (17) an ergodic PDE system with unknowns $(\lambda, \psi, \mu)$. Such PDE system expresses optimality conditions for the ergodic control problem corresponding to (10). It is easy to see that $\left(0,0, \mu_{\infty}\right)$ is a solution of that ergodic system. Therefore, we are addressing the same questions studied in the above mentioned articles. However, the equations we are looking at are quite different. A fundamental difference is that the coupling terms in (10) are not monotone in the sense of [6, Eq.(7) p. 8].

\subsubsection{Exponential convergence to equilibrium and the turnpike property}

A key step towards the forthcoming quantitative estimates is to consider the timereversed version of our mean field Schrödinger problem. For $Q \in \mathcal{P}(\Omega)$ the time reversal $\hat{Q}$ is the law of the time reversed process $\left(X_{T-t}\right)_{t \in[0, T]}$. In Lemma 4.5 we prove that if $\mathrm{P}$ is an optimizer for (MFSP), then $\hat{\mathrm{P}}$ optimizes

$$
\inf \left\{\mathcal{H}(\mathrm{Q} \mid \Gamma(\mathrm{Q})): \mathrm{Q} \in \mathcal{P}_{1}(\Omega), \mathrm{Q}_{0}=\mu^{\mathrm{fin}}, \mathrm{Q}_{T}=\mu^{\text {in }}\right\}
$$

The optimality of $\hat{\mathrm{P}}$ implies the existence of an associated process $\hat{\Psi}$ as described in Theorem 1.3. We show at Theorem 1.6 below that the function 


$$
[0, T] \ni t \mapsto \mathbb{E}_{\mathrm{P}}\left[\Psi_{t}\left(X_{t}\right) \cdot \hat{\Psi}_{T-t}\left(\hat{X}_{T-t}\right)\right]
$$

is a constant, that we denote $\mathscr{E}_{\mathrm{P}}\left(\mu^{\mathrm{in}}, \mu^{\mathrm{fin}}\right)$ and call the conserved quantity. Naturally this quantity depends also on $T$ but we omit this from the notation.

Theorem 1.4 confirms the intuition that mean field Schrödinger bridges are localized around $\mu_{\infty}$ providing an explicit upper bound for $\mathcal{F}\left(\mathrm{P}_{t}\right)$ along any MFSB, where

$$
\mathcal{F}(\mu)=\tilde{\mathcal{F}}(\mu)-\tilde{\mathcal{F}}\left(\mu_{\infty}\right) .
$$

We recall that $\mu_{\infty}$ is found by minimizing $\tilde{\mathcal{F}}$ among all elements of $\mathcal{P}_{2}\left(\mathbb{R}^{d}\right)$ whose mean is the same as $\mu$. If $\tilde{\mathcal{F}}$ is thought of as a free energy, then $\mathcal{F}$ should be thought of as a divergence (from equilibrium). A graphical illustration of Theorem 1.4 and the turnpike property is provided in the appendix.

Theorem 1.4 Assume (H1)-(H4) and let $\mathrm{P}$ be an optimizer for (MFSP). For all $t \in$ $[0, T]$ we have

$$
\begin{aligned}
\mathcal{F}\left(\mathrm{P}_{t}\right) \leq & \frac{\sinh (2 \kappa(T-t))}{\sinh (2 \kappa T)}\left(\mathcal{F}\left(\mu^{\mathrm{in}}\right)-\frac{\mathscr{E}_{\mathrm{P}}\left(\mu^{\mathrm{in}}, \mu^{\mathrm{fin}}\right)}{2 \kappa}\right) \\
& +\frac{\sinh (2 \kappa t)}{\sinh (2 \kappa T)}\left(\mathcal{F}\left(\mu^{\mathrm{fin}}\right)-\frac{\mathscr{E}_{\mathrm{P}}\left(\mu^{\mathrm{in}}, \mu^{\mathrm{fin}}\right)}{2 \kappa}\right)+\frac{\mathscr{E}_{\mathrm{P}}\left(\mu^{\mathrm{in}}, \mu^{\mathrm{fin}}\right)}{2 \kappa} .
\end{aligned}
$$

Moreover, for all fixed $\theta \in(0,1)$ there exists a decreasing function $B(\cdot)$ such that

$$
\mathcal{F}\left(\mathrm{P}_{\theta T}\right) \leq B(\kappa)\left(\mathcal{F}\left(\mu^{\mathrm{in}}\right)+\mathcal{F}\left(\mu^{\mathrm{fin}}\right)\right) \exp (-2 \kappa \min \{\theta, 1-\theta\} T)
$$

uniformly in $T \geq 1$.

In particular, since $\mathcal{F}\left(\mathrm{P}_{\theta T}\right)$ dominates $\mathcal{W}_{2}\left(\mathrm{P}_{\theta T}, \mu_{\infty}\right)$ (see e.g. [12, (ii), Thm 2.21 1]), we obtain that $\mathrm{P}_{\theta T}$ converges exponentially to $\mu_{\infty}$ with exponential rate proportional to $\kappa$. The proof of (22) is done by bounding the second derivative of the function $t \mapsto$ $\mathcal{F}\left(\mathrm{P}_{t}\right)$ along Schrödinger bridges with the help of the logarithmic Sobolev inequality established in [12]. To obtain (23) from (22) we use a functional inequality for the conserved quantity and a Talagrand inequality for $\mathscr{C}_{T}\left(\mu^{\text {in }}, \mu^{\text {fin }}\right)$, that are the content of Theorem 1.6 and Corollary 1.3 below. It is worth mentioning that the estimates (22), (23) (as well as (32) below) appear to be new even for the classical Schrödinger bridge problem and have not been anticipated by the heuristic articles [28,34]. Conversely, the above mentioned estimates admit a geometrical interpretation in the framework of Otto calculus that allows to formally extend their validity to the whole class of problems studied in [28].

Remark 1.4 The exponential rate in (23) has a sharp dependence on $\kappa$. To see this, fix $\mu^{\mathrm{in}}$ and choose $\mu^{\mathrm{fin}}=\mathrm{P}_{T}^{\mathrm{MKV}}$. Then it is easy to see that the restriction of $\mathrm{P}^{\mathrm{MKV}}$ to the interval $[0, T]$ is an optimizer for (MFSP). Setting $\theta=1 / 2$ and considering (23) for $T=2 t$ we arrive at

$$
\forall t \geq 1 / 2, \quad \mathcal{F}\left(\mathrm{P}_{t}^{\mathrm{MKv}}\right) \leq B(\kappa) \exp (-2 \kappa t)
$$


Thus, we obtain the same exponential rate as in $[12]^{5}$, that is easily seen to be optimal under the assumption that $W$ is $\kappa$-convex. A similar argument can be used to show the optimal dependence of the rate in $\theta$.

In the previous theorem we showed that, when looking at a timescale that is proportional to $T$, the marginal distribution of any Schrödinger bridge is exponentially close to $\mu_{\infty}$. Here we show that for a fixed value of $t$, we have an exponential convergence towards the law of the McKean-Vlasov dynamics $\mathrm{P}^{\mathrm{MKV}}$, see (5).

Theorem 1.5 Assume (H1)-(H4) and let $\mathrm{P}$ be an optimizer for (MFSP). For all $t \in$ $[0, T]$ we have

$$
\begin{aligned}
& \mathcal{W}_{2}^{2}\left(\mathrm{P}_{t}, \mathrm{P}_{t}^{M K V}\right) \\
& \leq 2 t\left(\frac{\mathcal{F}\left(\mu^{\mathrm{in}}\right)}{\exp (2 \kappa T)-1}+\frac{\exp (2 \kappa T)-\exp (2 \kappa(T-t))}{\exp (2 \kappa(T-t))-1} \frac{\mathcal{F}\left(\mu^{\mathrm{fin}}\right)}{\exp (2 \kappa T)-1}\right)
\end{aligned}
$$

In particular, the above theorem tells that $\mathcal{W}_{2}^{2}\left(\mathrm{P}_{t}, \mathrm{P}_{t}^{\mathrm{MKV}}\right)$ decays asymptotically at least as fast as $\exp (-2 \kappa T)$ when $T$ is large.

\subsubsection{Functional inequalities for the mean field entropic cost}

It is well known that analysing the evolution of entropy-like functionals along the socalled displacement interpolation of optimal transport has far reaching consequences in terms functional inequalities [55]. Since (MFSP) provides with an alternative way of interpolating between probability measures, it is tempting to see if it leads to new functional inequalities involving the cost $\mathscr{C}_{T}\left(\mu^{\text {in }}, \mu^{\text {fin }}\right)$. Here, we present a Talagrand and an HWI inequality that we used in order to study the long time behavior of MFSBs. They generalize their respective counterparts in [48], [42]. Both inequalities are based on another upper bound for the evolution of $\mathcal{F}$ along MFSBs, whose presentation we postpone to Theorem 4.1.

The following Talagrand inequality tells that the mean field entropic cost grows at most linearly with $\mathcal{F}$ :

Corollary 1.3 (A Talagrand inequality) Assume (H1)-(H4). Then for all $T>0$ we have

$$
\begin{aligned}
\forall t \in(0, T), \quad \mathscr{C}_{T}\left(\mu^{\mathrm{in}}, \mu^{\mathrm{fin}}\right) \leq & \frac{1}{\exp (2 \kappa t)-1} \mathcal{F}\left(\mu^{\mathrm{in}}\right) \\
& +\frac{\exp (2 \kappa(T-t))}{\exp (2 \kappa(T-t))-1} \mathcal{F}\left(\mu^{\mathrm{fin}}\right) .
\end{aligned}
$$

In particular, choosing $\mu^{\text {fin }}=\mu_{\infty}$ leads to

$$
\mathscr{C}_{T}\left(\mu^{\text {in }}, \mu_{\infty}\right) \leq \frac{1}{\exp (2 \kappa T)-1} \mathcal{F}\left(\mu^{\text {in }}\right) .
$$

\footnotetext{
5 Some doubt on the numeric value of the exponential rates may arise from the fact that in our definition of $\tilde{\mathcal{F}}$, there is no $1 / 2$ in front of $W$, as it is the case in [12, Eq. 1.3]. However, as we pointed out before, the McKean-Vlasov dynamics for the particle system (1) is the gradient flow of $1 / 2 \tilde{\mathcal{F}}$ and not of $\tilde{\mathcal{F}}$.
} 
Unlike the classical case, in the entropic HWI inequality the Wasserstein distance is replaced by the conserved quantity $\mathscr{E} P$ in the first term on the rhs and by the mean field entropic cost in the second term. An extra positive contribution $\frac{1}{4} \mathcal{I}_{\mathcal{F}}$ is present in the first term. Our interpretation is that this compensates for the fact that in the "gain" term we put the $\operatorname{cost} \mathscr{C}_{T}$, that is larger than the squared Wasserstein distance. In order to state the HWI inequality, we introduce the non linear Fisher information functional $\mathcal{I}_{\mathcal{F}}$ defined for $\mu \in \mathcal{P}_{2}\left(\mathbb{R}^{d}\right)$ by

$$
\mathcal{I}_{\mathcal{F}}(\mu)= \begin{cases}\int_{\mathbb{R}^{d}}|\nabla \log \mu+2 \nabla W * \mu(x)|^{2} \mu(\mathrm{d} x), & \text { if } \nabla \log \mu \in L_{\mu}^{2} \\ +\infty & \text { otherwise. }\end{cases}
$$

where by $\nabla \log \mu \in L_{\mu}^{2}$ we mean $\mu \ll \lambda$ and that $\log \mu$ is an absolutely continuous function on $\mathbb{R}^{d}$ whose derivative is in $L_{\mu}^{2}$. The non linear Fisher information can be seen to be equal to the derivative of the free energy $\tilde{\mathcal{F}}$ along the marginal flow of the McKean Vlasov dynamics.

Corollary 1.4 (An HWI inequality) Assume $(\mathrm{H} 1)-(\mathrm{H} 3)$ and choose $\mu^{\mathrm{fin}}=\mu_{\infty}$. If $\mathrm{P}$ is an optimizer for (MFSP) and $t \mapsto \mathcal{I}_{\mathcal{F}}\left(\mathrm{P}_{t}\right)$ is continuous ${ }^{6}$ in a right neighbourhood of 0 , then

$$
\begin{aligned}
\mathcal{F}\left(\mu^{\mathrm{in}}\right) \leq & \frac{1-\exp (-2 \kappa T)}{2 \kappa}\left(\mathcal{I}_{\mathcal{F}}\left(\mu^{\mathrm{in}}\right)\left(\frac{1}{4} \mathcal{I}_{\mathcal{F}}\left(\mu^{\mathrm{in}}\right)-\mathscr{E}_{\mathrm{P}}\left(\mu^{\mathrm{in}}, \mu_{\infty}\right)\right)\right)^{1 / 2} \\
& -(1-\exp (-2 \kappa T)) \mathscr{C}_{T}\left(\mu^{\mathrm{in}}, \mu_{\infty}\right) .
\end{aligned}
$$

It is worth noticing that by letting $T \rightarrow+\infty$ in the above HWI inequality we obtain the logarithmic Sobolev inequality [12, Thm 2.2]. Indeed, $\mathscr{C}_{T}\left(\mu^{\mathrm{in}}, \mu_{\infty}\right)$ is always non negative and we shall see at Theorem 1.6 below that $\mathscr{E}_{\mathrm{P}}\left(\mu^{\mathrm{in}}, \mu_{\infty}\right) \rightarrow 0$. The short time regime is also interesting. Indeed, if $W=0, \mathscr{C}_{T}\left(\mu^{\text {in }}, \mu_{\infty}\right)$ is the standard entropic cost and we have under suitable hypothesis on $\mu^{\text {in }}$ (see [40])

$$
\lim _{T \rightarrow 0} T \mathscr{C}_{T}\left(\mu^{\mathrm{in}}, \mu_{\infty}\right)=\frac{1}{2} \mathcal{W}_{2}^{2}\left(\mu^{\mathrm{in}}, \mu_{\infty}\right) .
$$

The heuristic arguments put forward in [28] tell that (29) is expected to be true even when $W$ is a general potential satisfying (H1). Following again (29), one also expects that

$$
\lim _{T \rightarrow 0} \frac{T^{2}}{4} \mathcal{I}_{\mathcal{F}}\left(\mu^{\text {in }}\right)-T^{2} \mathscr{E}_{\mathrm{P}}\left(\mu^{\text {in }}, \mu_{\infty}\right)=\mathcal{W}_{2}^{2}\left(\mu^{\text {in }}, \mu_{\infty}\right)
$$

Putting (29) and (30) together we obtain an heuristic justification of the fact that in the limit as $T \rightarrow 0$ (28) becomes the classical HWI inequality put forward in [12],

\footnotetext{
6 We were not able to conclude that in general (H1) and (H2) imply this, although we could establish the continuity of $\mathcal{I}_{\mathcal{F}}\left(\mathrm{P}_{t}\right)$ on any open subinterval of $[0, T]$.
} 
namely

$$
\mathcal{F}\left(\mu^{\text {in }}\right) \leq \mathcal{W}_{2}\left(\mu^{\text {in }}, \mu_{\infty}\right) \mathcal{I}_{\mathcal{F}}\left(\mu^{\text {in }}\right)^{1 / 2}-\kappa \mathcal{W}_{2}^{2}\left(\mu^{\text {in }}, \mu_{\infty}\right)
$$

Our last result is a functional inequality that establishes a hierarchical relation between the conserved quantity and the mean field entropic cost: the former is exponentially small in $T$ and $\kappa$ in comparison with the latter. We may refer to this as an energy-transport inequality since the conserved quantity may be geometrically interpreted as the total energy of a physical system (cf. [17, Corollary 1.1]).

Theorem 1.6 Assume (H1)-(H4) and let $\mathrm{P}$ be an optimizer. Then the function

$$
[0, T] \ni t \mapsto \mathbb{E}_{\mathrm{P}}\left[\Psi_{t}\left(X_{t}\right) \cdot \hat{\Psi}_{T-t}\left(\hat{X}_{T-t}\right)\right]
$$

is constant. Denoting this constant by $\mathscr{E}\left(\mu^{\mathrm{in}}, \mu^{\text {fin }}\right)$, we have

$$
\left|\mathscr{E}_{\mathrm{P}}\left(\mu^{\mathrm{in}}, \mu^{\mathrm{fin}}\right)\right| \leq \frac{4 \kappa}{\exp (\kappa T)-1}\left(\mathscr{C}_{T}\left(\mu^{\mathrm{in}}, \mu^{\mathrm{fin}}\right) \mathscr{C}_{T}\left(\mu^{\mathrm{fin}}, \mu^{\mathrm{in}}\right)\right)^{1 / 2}
$$

In general the term $\mathscr{C}_{T}\left(\mu^{\mathrm{in}}, \mu^{\mathrm{fin}}\right) \mathscr{C}_{T}\left(\mu^{\mathrm{fin}}, \mu^{\mathrm{in}}\right)$ in (32) cannot be simplified further, since typically $\mathscr{C}_{T}\left(\mu^{\mathrm{in}}, \mu^{\mathrm{fin}}\right) \neq \mathscr{C}_{T}\left(\mu^{\mathrm{fin}}, \mu^{\mathrm{in}}\right)$. E.g. $\mathscr{C}_{T}\left(\delta_{0}, v\right)=0$ if $v$ is the law of the unconstrained McKean-Vlasov SDE at time $T$ started at zero, whereas $\mathscr{C}_{T}\left(v, \delta_{0}\right)>0$, as it takes effort to drive such SDE to zero.

\section{Connections with optimal transport}

In this section we shall see how the results of this article relate to the Riemannian calculus on $\mathcal{P}_{2}\left(\mathbb{R}^{d}\right)$ introduced by Otto [41], at least formally. The reader not interested in optimal transport per se is encouraged to skip this section in a first reading. The link is rooted in a seemingly novel connection between (McKean-Vlasov) FBSDEs and second order ODEs in the Riemannian manifold of optimal transport that we find of independent interest. To better understand this connection, let us begin by recalling that in the seminal article [31] it is proven that the marginal flow of the trajectorial SDE

$$
\mathrm{d} X_{t}=-\nabla U\left(X_{t}\right) \mathrm{d} t+\mathrm{d} B_{t}
$$

can be interpreted as the gradient flow of the entropy functional

$$
\mu \mapsto \frac{1}{2} \int_{\mathbb{R}^{d}} \log \mu(x) \mu(\mathrm{d} x)+\int_{\mathbb{R}^{d}} U(x) \mu(\mathrm{d} x)
$$

w.r.t. the 2-Wasserstein metric. Thus, first order Itô SDEs provide with probabilistic representations for first order ODEs in the Riemannian manifold of optimal transport. Of course, since a path measure is not fully determined by its one time marginals, 
the SDE (33) contains more information than the gradient flow equation. It has been shown in [17] that the marginal flow of a classical Schrödinger bridge satisfies a second order ODE, more precisely a Newton's law in which the acceleration field is the Wasserstein gradient of the Fisher information functional. The natural question is then: What trajectorial (second order) SDE governs the dynamics of a Schrödinger bridge and yields a probabilistic representation for the associated Newton's law? In order to answer this, let us first recall some notions of Otto calculus.

\subsection{Second order calculus on $\mathcal{P}_{2}\left(\mathbb{R}^{d}\right)$}

In the next lines, we sketch the ideas behind the Riemannian calculus on $\mathcal{P}_{2}\left(\mathbb{R}^{d}\right)$. It would be impossible to provide a self-contained introduction in this work and we refer to [53] or [29] for detailed accounts. The main idea is to equip $\mathcal{P}_{2}\left(\mathbb{R}^{d}\right)$ with a Riemannian metric such that the associated geodesic distance is $\mathcal{W}_{2}(\cdot, \cdot)$. To do this, one begins by identifying the tangent space $\mathcal{T}_{\mu} \mathcal{P}_{2}$ at $\mu \in \mathcal{P}_{2}\left(\mathbb{R}^{d}\right)$ as the space closure in $L_{\mu}^{2}$ of the subspace of gradient vector fields

$$
\mathcal{T}_{\mu} \mathcal{P}_{2}=\overline{\left\{\nabla \varphi, \varphi \in \mathcal{C}_{c}^{\infty}\left(\mathbb{R}^{d}\right)\right\}} L_{\mu}^{2}
$$

The velocity (first derivative) of a sufficiently regular curve $[0, T] \ni t \mapsto \mu_{t} \in \mathcal{P}_{2}\left(\mathbb{R}^{d}\right)$ is then defined by looking at the only solution $v_{t}(x)$ of the continuity equation

$$
\partial_{t} \mu_{t}+\nabla \cdot\left(v_{t} \mu_{t}\right)=0
$$

such that $v_{t} \in \mathcal{T}_{\mu_{t}} \mathcal{P}_{2}$ for all $t \in[0, T]$. Finally, the Riemannian metric (Otto metric) $\langle\cdot, \cdot\rangle_{\mathcal{T}_{\mu}} \mathcal{P}_{2}$ is defined by

$$
\langle\nabla \varphi, \nabla \psi\rangle_{\mathcal{T}_{\mu} \mathcal{P}_{2}}=\int_{\mathbb{R}^{d}} \nabla \varphi \cdot \nabla \psi(x) \mu(\mathrm{d} x) .
$$

It can be seen that the constant speed geodesic curves associated to the Riemannian metric we have introduced coincide with the displacement interpolations of optimal transport and that the corresponding geodesic distance is indeed $\mathcal{W}_{2}(\cdot, \cdot)$. This makes it possible to carry out several explicit calculations. In particular, we can compute the gradient $\operatorname{grad}^{\mathcal{W}} \mathcal{F}$ and the Hessian Hess $\mathcal{W}_{2} \mathcal{F}$ of a smooth functional $\mathcal{F}: \mathcal{P}_{2}\left(\mathbb{R}^{d}\right) \rightarrow$ $\mathbb{R}$. At least formally, we have

$$
\begin{aligned}
\left\langle\operatorname{grad}^{\mathcal{W}} \mathcal{F}, \nabla \varphi\right\rangle_{\mathcal{T}_{\mu}} \mathcal{P}_{2} & =\left.\frac{\mathrm{d}}{\mathrm{d} h} \mathcal{F}\left((i d+h \nabla \varphi)_{\#} \mu\right)\right|_{h=0} \\
\left\langle\nabla \varphi, \operatorname{Hess}_{\mu} \mathcal{W}_{2} \mathcal{F}(\nabla \varphi)\right\rangle_{\mathcal{T}_{\mu} \mathcal{P}_{2}} & =\left.\frac{\mathrm{d}^{2}}{\mathrm{~d}^{2}} \mathcal{F}\left((i d+h \nabla \varphi)_{\#} \mu\right)\right|_{h=0},
\end{aligned}
$$

where we used the notation \# for the push forward. In particular, setting $W=0$ for simplicity in (27) we obtain that the classical Fisher information functional $\mathcal{I}$ has a gradient that can be computed with the rules above. One obtains that (cf. [54]) 


$$
\operatorname{grad}^{\mathcal{W}_{2}} \mathcal{I}(\mu)=-2 \nabla \Delta \log \mu-\nabla|\nabla \log \mu|^{2}
$$

The Levi-Civita connection associated to the Riemannian metric (34) can also be explicitly computed with the help of the orthogonal projection operator $\Pi_{\mu}: L_{\mu}^{2} \rightarrow$ $\mathcal{T}_{\mu} \mathcal{P}_{2}$. To do this, consider a regular curve $\left(\mu_{t}\right)_{t \in[0, T]}$ with velocity $\left(v_{t}\right)_{t \in[0, T]}$ and a tangent vector field $t \mapsto u_{t} \in \mathcal{T}_{\mu_{t}} \mathcal{P}_{2}$ along $\left(\mu_{t}\right)_{t \in[0, T]}$. It turns out that if one defines the covariant derivative $\frac{\mathbf{D}}{\mathrm{d} t} u_{t}$ of $\left(u_{t}\right)_{t \in[0, T]}$ along $\left(\mu_{t}\right)_{t \in[0, T]}$ as the vector field

$$
\frac{\mathbf{D}}{\mathrm{d} t} u_{t}=\Pi_{\mu_{t}}\left(\partial_{t} u_{t}+\mathrm{D} u_{t} \cdot v_{t}\right)
$$

then this covariant derivative satisfies the compatibility with the metric and the torsionfree identity, i.e. it is the Levi-Civita connection. The acceleration of the curve $\left(\mu_{t}\right)_{t \in[0, T]}$ is then the covariant derivative of the velocity along the curve, i.e.

$$
\frac{\mathbf{D}}{\mathrm{d} t} v_{t}=\partial_{t} v_{t}+\frac{1}{2} \nabla\left|v_{t}\right|^{2}
$$

\subsection{Newton's laws and FBSDEs}

According to the above discussion the Newton's law in $\left(\mathcal{P}_{2}\left(\mathbb{R}^{d}\right),\langle., .\rangle_{\mathcal{T} \mathcal{P}_{2}}\right)$

$$
\left\{\begin{array}{l}
\frac{\mathbf{D}}{\mathrm{d} t} v_{t}=\frac{1}{8} \operatorname{grad}^{\mathcal{W}_{2}} \mathcal{I}\left(\mu_{t}\right) \\
\mu_{0}=\mu^{\mathrm{in}}, \mu_{T}=\mu^{\mathrm{fin}}
\end{array}\right.
$$

provides with a geometrical interpretation for the PDE system (see [17] for more details)

$$
\left\{\begin{array}{l}
\partial_{t} \mu_{t}(x)+\nabla \cdot\left(\nabla \phi_{t}(x) \mu_{t}(x)\right)=0 \\
\partial_{t} \nabla \phi_{t}(x)+\frac{1}{2} \nabla\left|\nabla \phi_{t}(x)\right|^{2}=-\frac{1}{4} \nabla \Delta \log \mu_{t}(x)-\frac{1}{8} \nabla\left|\log \mu_{t}(x)\right|^{2} \\
\mu_{0}=\mu^{\text {in }}, \mu_{T}=\mu^{\mathrm{fin}}
\end{array}\right.
$$

where to derive the latter equation we observe that the requirement that $v_{t} \in \mathcal{T}_{\mu_{t}} \mathcal{P}_{2}$ for all $t \in[0, T]$ is formally equivalent to $v_{t}=\nabla \phi_{t}$ for some time dependent potential $(t, x) \mapsto \phi_{t}(x)$.

As we have seen in Sect. 1.3.1, solutions of the FBSDE

$$
\left\{\begin{array}{l}
\mathrm{d} X_{t}=Y_{t} \mathrm{~d} t+\mathrm{d} B_{t} \\
\mathrm{~d} Y_{t}=Z_{t} \cdot \mathrm{d} B_{t} \\
X_{0} \sim \mu^{\text {in }}, X_{T} \sim \mu^{\mathrm{fin}}
\end{array}\right.
$$


having the additional property that $Y_{t}=\nabla \psi_{t}\left(X_{t}\right)$ yield a probabilistic representation for

$$
\left\{\begin{array}{l}
\partial_{t} \mu_{t}(x)-\frac{1}{2} \Delta \mu_{t}(x)+\nabla \cdot\left(\nabla \psi_{t}(x) \mu_{t}(x)\right)=0, \\
\partial_{t} \nabla \psi_{t}(x)+\frac{1}{2} \nabla \Delta \psi_{t}(x)+\nabla^{2} \psi_{t}(x) \cdot \nabla \psi_{t}(x)=0, \\
\mu_{0}(x)=\mu^{\mathrm{in}}(x), \mu_{T}(x)=\mu^{\mathrm{fin}}(x) .
\end{array}\right.
$$

Some tedious though standard calculations allow to see that the change of variable $\phi_{t}=-\frac{1}{2} \log \mu_{t}+\psi_{t}$ transforms the PDE system (39) in (37). Summing up, we have obtained the following

Informal statement We have:

(i) If $\left(X_{t}, Y_{t}, Z_{t}\right)_{t \in[0, T]}$ is a solution for the FBSDE (38) such that $Y_{t}=\nabla \psi_{t}\left(X_{t}\right)$ for some time-varying potential $\psi$, then the marginal flow $\left(\mu_{t}\right)_{t \in[0, T]}$ of $X_{t}$ is a solution for the Newton's law (36).

(ii) If $\mathrm{P}$ is the (classical) Schrödinger bridge between $\mu^{\mathrm{in}}$ and $\mu^{\mathrm{fin}}$, then under $\mathrm{P}$ the canonical process $\left(X_{t}\right)_{t \in[0, T]}$ is such that there exist processes $\left(Y_{t}\right)_{t \in[0, T]}$, $\left(Z_{t}\right)_{t \in[0, T]}$ with the property that $\left(X_{t}, Y_{t}, Z_{t}\right)_{t \in[0, T]}$ is a solution for (38) and $Y_{t}$ is as in (i)

We leave it to future work to prove a rigorous version of the informal statement above. On the formal level, there is no conceptual difficulty in extending it to include the interaction potential $W$. Essentially, the only difference is that one has to deal with the non linear Fisher information functional $\mathcal{I}_{\mathcal{F}}$ instead of $\mathcal{I}$.

Beside its intrinsic interest, the parallelism between Newton's laws and FBSDEs is very useful when studying the long time behavior of the latter. Indeed, the Riemannian structure underlying (36) allows to find tractable expressions for the first and second derivative of entropy-like functionals along the marginal flow of the FBSDE.

Remark 2.1 Classical Schrödinger bridges are $h$-transforms in the sense of Doob [22]. Therefore, one can also describe their dynamics with a first order SDE and a PDE that encodes the evolution of the drift field. This is not strictly speaking a probabilistic representation of (36) since there is already a PDE involved. Our FBSDE approach may be viewed as a way to interpret in a trajectorial sense the PDE governing the drift in the $h$-transform representation.

\section{The mean field Schrödinger problem and its equivalent formulations: proofs}

In this part we complement the discussion undertaken in Sect. 1.2 and provide the proofs of the results stated therein. This section is organized into four subsections so that

- Section 3.1 contains the proof of Theorem 3.1, which generalizes Theorem 1.1, along with several useful lemmas, 
- Section 3.2 is where we prove Proposition 1.1, Lemma 1.1 and Theorem 1.3.

- Theorem 1.2 is proven in Sect. 3.3.

- Finally, Corollary 1.1 and 1.2 are proven in Sect. 3.4.

In the whole section, apart from Sect. 3.1 that has its own assumptions, we always assume that $(\mathrm{H} 1),(\mathrm{H} 2)$ are in force, even if we do not write them down explicitly in the statements of the lemmas and propositions.

\subsection{A large deviations principle for particles interacting through their drifts}

We consider for $N \in \mathbb{N}$ the interacting particle system

$$
\left\{\begin{aligned}
\mathrm{d} X_{t}^{i, N} & =\frac{1}{N} \sum_{k=1}^{N} b\left(t, X^{i, N}, X^{k, N}\right) \mathrm{d} t+\mathrm{d} B_{t}^{i} \\
X_{0}^{i, N} & \sim \mu^{\mathrm{in}}, \quad i=1, \ldots, N .
\end{aligned}\right.
$$

where $\left\{B^{i}: i=1, \ldots, N\right\}$ are independent Brownian motions and $\left\{X_{0}^{i, N}: i=\right.$ $1, \ldots, N\}$ are independent to each other and to the Brownian motions. Regarding the drift $b$, we assume

$$
\begin{aligned}
& {[0, T] \times \Omega \times \Omega \ni(t, \omega, \bar{\omega}) \mapsto b(t, \omega, \bar{\omega}) \in \mathbb{R}^{d} \text { is progressively measurable, }} \\
& \left|b\left(t, \omega^{1}, \bar{\omega}^{1}\right)-b\left(t, \omega^{2}, \bar{\omega}^{2}\right)\right| \leq C\left\{\sup _{s \leq t}\left|\omega_{s}^{1}-\omega_{s}^{2}\right|+\sup _{s \leq t}\left|\bar{\omega}_{s}^{1}-\bar{\omega}_{s}^{2}\right|\right\} \\
& \int_{0}^{T}|b(s, 0,0)| \mathrm{d} s \leq C,
\end{aligned}
$$

for some constant $C>0$ and all $\left(t, \omega^{1}, \omega^{2}, \bar{\omega}^{1}, \bar{\omega}^{2}\right) \in[0, T] \times \Omega^{4}$. Finally, regarding the measure $\mu^{\text {in }}$ we assume that

$$
\int_{\mathbb{R}^{d}} \exp \left(r|x|^{\beta}\right) \mu^{\text {in }}(\mathrm{d} x)<\infty \text { for all } r>0
$$

We stress that the usual theory of stochastic differential equations guarantees the strong existence and uniqueness for the above interacting particle system. Furthermore, if $\mathrm{P} \in \mathcal{P}_{1}(\Omega)$ then the same arguments show that the stochastic differential equation

$$
\left\{\begin{aligned}
\mathrm{d} X_{t}^{\mathrm{P}} & =\left[\int b\left(t, X^{\mathrm{P}}, \bar{\omega}\right) \mathrm{P}(\mathrm{d} \bar{\omega})\right] \mathrm{d} t+\mathrm{d} B_{t} \\
X_{0}^{\mathrm{P}} & \sim \mu^{\mathrm{in}},
\end{aligned}\right.
$$

admits a unique strong solution. We denote $\Gamma(\mathrm{P})$ the law of $X^{\mathrm{P}}$. We can now state the main result of this part, which contains Theorem 1.1 as a very particular case. 
Theorem 3.1 Let $\beta \in[1,2)$ and assume (40), (41), (42), (43). Then the sequence of empirical measures

$$
\left\{\frac{1}{N} \sum_{i=1}^{N} \delta_{X^{i, N}}: N \in \mathbb{N}\right\}
$$

satisfies a LDP on $\mathcal{P}_{\beta}(\Omega)$ equipped with the $\mathcal{W}_{\beta}$-topology, with good rate function given by

$$
\mathcal{P}_{\beta}(\Omega) \ni \mathrm{P} \mapsto \mathscr{I}(\mathrm{P}):= \begin{cases}\mathcal{H}(\mathrm{P} \mid \Gamma(\mathrm{P})), & \mathrm{P} \ll \Gamma(\mathrm{P}) \\ +\infty, & \text { otherwise. }\end{cases}
$$

The result is sharp, in that it fails for $\beta=2$; see [56]. We follow Tanaka's reasoning [49] in order to establish this large deviations result. We remark that the assumption on exponential moments (43) is only used in the proof of Theorem 3.1, and not in the results preceding this proof.

For $\mathrm{Q} \in \mathcal{P}_{\beta}(\Omega)$ we consider the equation

$$
Y_{t}(\omega)=\omega_{t}+\int_{0}^{t}\left[\int b(s, Y(\omega), Y(\bar{\omega})) \mathrm{Q}(\mathrm{d} \bar{\omega})\right] \mathrm{d} s
$$

Lemma 3.1 Take $Y_{t}^{(0)}(\omega):=\omega_{0}, \mathrm{Q} \in \mathcal{P}_{\beta}(\Omega)$, and consider the iterations

$$
Y_{t}^{(n+1)}(\omega)=\omega_{t}+\int_{0}^{t}\left[\int b\left(s, Y^{(n)}(\omega), Y^{(n)}(\bar{\omega})\right) \mathrm{Q}(\mathrm{d} \bar{\omega})\right] \mathrm{d} s, \quad s \leq T .
$$

Then

(a) The iteration is well-defined $\omega$-by- $\omega$ (in particular, the Q-integrals are welldefined and finite) and in fact $\sup _{n} \mathbb{E}_{\mathrm{Q}}\left[\sup _{t \leq T}\left|Y_{t}^{n}\right|^{\beta}\right]$ is finite.

(b) For each $\omega \in \Omega$ the sequence $\left\{Y^{(n)}(\omega)\right\}_{n \in \mathbb{N}}$ is convergent in the sup-norm to some limiting continuous path $Y^{(\infty)}(\omega)$. Further $\mathbb{E}_{\mathrm{Q}}\left[\sup _{t \leq T}\left|Y_{t}^{(\infty)}\right|^{\beta}\right]<\infty$, $\mathbb{E}_{\mathrm{Q}}\left[\sup _{t \leq T}\left|Y_{t}^{(\infty)}-Y_{t}^{(n)}\right|\right] \rightarrow 0$, and $Y^{(\infty)}$ is adapted to the canonical filtration.

Proof From the Lipschitz assumption on $b$ we first derive

$$
\begin{aligned}
\sup _{s \leq t}\left|Y_{s}^{(n+1)}\right| \leq & \sup _{s \leq t}\left|\omega_{s}\right|+\int_{0}^{T}|b(s, 0,0)| \mathrm{d} s+C \int_{0}^{t} \sup _{r \leq s}\left|Y_{r}^{(n)}\right| \mathrm{d} r \\
& +C \int_{0}^{t} \mathbb{E}_{\mathrm{Q}}\left[\sup _{r \leq s}\left|Y_{r}^{(n)}\right|\right] \mathrm{d} r .
\end{aligned}
$$

Raising this to $\beta$, taking expectations and using Jensen's inequality, we derive

$$
\mathbb{E}_{\mathrm{Q}}\left[\sup _{s \leq t}\left|Y_{s}^{(n+1)}\right|^{\beta}\right] \leq C^{\prime}\left(1+\mathbb{E}_{\mathrm{Q}}\left[\sup _{s \leq T}\left|\omega_{s}\right|^{\beta}\right]+\int_{0}^{t} \mathbb{E}_{\mathrm{Q}}\left[\sup _{r \leq s}\left|Y_{r}^{(n)}\right|^{\beta}\right] \mathrm{d} r\right)
$$


where $C^{\prime}$ only depends on $T$ and $\beta$. From this we establish for some $R \geq 0$ that

$$
\sup _{n} \mathbb{E}_{\mathrm{Q}}\left[\sup _{s \leq t}\left|Y_{s}^{(n)}\right|^{\beta}\right] \leq R e^{R t}
$$

Now denote $\Delta_{t}^{n}:=\sup _{s \leq t}\left|Y_{s}^{(n)}-Y_{s}^{(n-1)}\right|$. Again by the Lipschitz property

$$
\Delta_{t}^{n+1} \leq C \int_{0}^{t}\left\{\Delta_{s}^{n}+\mathbb{E}_{\mathrm{Q}}\left[\Delta_{s}^{n}\right]\right\} \mathrm{d} s
$$

which we can bootstrap to obtain

$$
\Delta_{t}^{n+1}+\mathbb{E}_{\mathrm{Q}}\left[\Delta_{t}^{n+1}\right] \leq 3 C \int_{0}^{t}\left\{\Delta_{s}^{n}+\mathbb{E}_{\mathrm{Q}}\left[\Delta_{s}^{n}\right]\right\} \mathrm{d} s .
$$

Observe that $\Delta_{t}^{1} \leq 2 \sup _{s<T}\left|\omega_{s}-\omega_{0}\right|+C$, so from the above inequality we obtain by induction that $\Delta_{t}^{n+1}+\mathbb{E}_{\mathrm{Q}}\left[\Delta_{t}^{n+1}\right] \leq C^{\prime \prime} \frac{t^{n}}{n !}$. From this $\left\{\Delta_{T}^{n}+\mathbb{E}_{\mathrm{Q}}\left[\Delta_{T}^{n}\right]\right\}_{n \in \mathbb{N}}$ is (for each $\omega$ ) summable in $n$, so the same happens to $\left\{\Delta_{T}^{n}\right\}_{n \in \mathbb{N}}$ and therefore the uniform limit of the $Y^{(n)}$ exists for all $\omega$. We denote by $Y^{(\infty)}$ this limit. By Fatou's lemma $\mathbb{E}_{\mathrm{Q}}\left[\sup _{t \leq T}\left|Y_{t}^{(\infty)}\right|^{\beta}\right]<\infty$. Since $\left(\mathbb{E}_{\mathrm{Q}}\left[\Delta_{T}^{n}\right]\right)_{n \in \mathbb{N}}$ is summable we must also have $\mathbb{E}_{\mathrm{Q}}\left[\sup _{t \leq T}\left|Y_{t}^{(\infty)}-Y_{t}^{(n)}\right|\right] \rightarrow 0$. Since clearly each $Y^{(n)}$ is adapted so is $Y^{(\infty)}$ too.

Lemma 3.2 For any $\mathrm{Q} \in \mathcal{P}_{\beta}$ there exists a unique adapted continuous process satisfying (45) pointwise. Denoting $Y^{\mathrm{Q}}$ this process, we further have

$$
\mathrm{Q} \circ\left(Y^{\mathrm{Q}}\right)^{-1} \in \mathcal{P}_{\beta}(\Omega)
$$

Proof If $X$ and $Y$ are solutions, then the Lipschitz assumption on $b$ implies

$$
\mathbb{E}_{\mathrm{Q}}\left[\sup _{s \leq t}\left|Y_{s}-X_{s}\right|\right] \leq K \int_{0}^{t} \mathbb{E}_{\mathrm{Q}}\left[\sup _{r \leq s}\left|Y_{r}-X_{r}\right|\right] \mathrm{d} r,
$$

so from Grönwall we derive $\mathbb{E}_{\mathrm{Q}}\left[\sup _{s \leq T}\left|Y_{s}-X_{s}\right|\right]=0$. With this, and using again the Lipschitz assumption on $b$, we find

$$
\sup _{s \leq t}\left|Y_{s}-X_{s}\right| \leq K \int_{0}^{t} \sup _{r \leq s}\left|Y_{r}-X_{r}\right| \mathrm{d} r
$$

so by Grönwall we deduce that $X=Y$ pointwise. For the existence of a solution we employ Point (b) of Lemma 3.1, taking limits in the iterations therein (the exchange of limit and integral is justified by the Lipschitz property of $b$ ). Finally $\mathrm{Q} \circ\left(Y^{\mathrm{Q}}\right)^{-1} \in$ $\mathcal{P}_{\beta}(\Omega)$ follows by Point (b) of Lemma 3.1 too. 
Thanks to this result we can define the operator

$$
\begin{aligned}
\Theta:\left(\mathcal{P}_{\beta}, \mathcal{W}_{\beta}\right) \rightarrow\left(\mathcal{P}_{\beta}, \mathcal{W}_{\beta}\right) \\
\mathrm{Q} \mapsto \Theta(\mathrm{Q}):=\mathrm{Q} \circ\left(Y^{\mathrm{Q}}\right)^{-1},
\end{aligned}
$$

where $Y^{\mathrm{Q}}$ denotes the unique solution of (45).

Lemma 3.3 $Y^{\mathrm{R}^{\mu^{\text {in }}}}$ is the unique strong solution to the McKean-Vlasov SDE

$$
\left\{\begin{aligned}
\mathrm{d} Z_{t} & =\left[\int b(t, Z, \bar{\omega}) \mathrm{P}(\mathrm{d} \bar{\omega})\right] \mathrm{d} t+\mathrm{d} B_{t} \\
Z & \sim \mathrm{P}, Z_{0} \sim \mu^{\mathrm{in}}
\end{aligned}\right.
$$

Furthermore, if $\left\{X^{i, N}: i \leq N, N \in \mathbb{N}\right\}$ is the aforementioned interacting particle system, which is driven by $\left\{B^{i}: i \in \mathbb{N}\right\}$ independent Brownian motions started like $\mu^{\text {in }}$, then

$$
\Theta\left(\frac{1}{N} \sum_{i=1}^{N} \delta_{B^{i}}\right)=\frac{1}{N} \sum_{i=1}^{N} \delta_{X^{i, N}}, \text { a.s. }
$$

Proof That $Y^{\mathrm{R}^{\mu^{\mathrm{in}}}}$ is a solution to the McKean-Vlasov SDE is clear since $\omega$ is a Brownian motion under $\mathrm{R}^{\mu \text { in }}$. That the solution is unique follows by observing that the drift in this SDE is Lipschitz jointly in $Z$ and $P=\operatorname{Law}(Z)$, from where usual arguments apply. For the second point, consider first $\omega^{1}, \ldots, \omega^{N}$ continuous paths and define $\mathrm{Q}=\frac{1}{N} \sum_{i=1}^{N} \delta_{\omega^{i}}$. Then for all $1 \leq i \leq N$ we have

$$
Y_{t}^{\mathrm{Q}}\left(\omega^{i}\right)=\omega_{t}^{i}+\int_{0}^{t}\left(\frac{1}{N} \sum_{k \leq N} b\left(s, Y^{\mathrm{Q}}\left(\omega^{i}\right), Y^{\mathrm{Q}}\left(\omega^{k}\right)\right)\right) \mathrm{d} s .
$$

Replacing the deterministic paths $\omega^{1}, \ldots, \omega^{N}$ by those of $B^{1}, \ldots, B^{N}$ we conclude.

The key observation is that $\frac{1}{N} \sum_{i=1}^{N} \delta_{B^{i}}$ satisfies a large deviations principle in $\mathcal{P}_{\beta}(\Omega)$ equipped with the $\mathcal{W}_{\beta}$ topology, with good rate function given by the relative entropy $\mathcal{H}\left(\cdot \mid \mathrm{R}^{\mu^{\text {in }}}\right)$. This is true for $\beta<2$ under our exponential moments assumption (43), but fails for $\beta=2$, as follows easily from [56]. By Lemma 3.3 we may derive, via the contraction principle ([21, Theorem 4.2.1]) a large deviations principle for

$$
\left\{\frac{1}{N} \sum_{i=1}^{N} \delta_{X^{i, N}}: N \in \mathbb{N}\right\}
$$

if we could only establish the continuity of $\Theta$. This is our next step.

Lemma 3.4 $\Theta$ is Lipschitz-continuous and injective. 
Proof We first prove the Lipschitz property. Let $\pi$ be a coupling with first marginal Q and second marginal P. Denoting $(\omega, \bar{\omega})$ the canonical process on $\Omega \times \Omega$, and by the Lipschitz assumption on $b$, we have

$$
\begin{aligned}
\mathbb{E}_{\pi}\left[\sup _{s \leq t}\left|Y_{s}^{\mathrm{Q}}(\omega)-Y_{s}^{\mathrm{P}}(\bar{\omega})\right|^{\beta}\right] \leq & K \int_{0}^{t} \mathbb{E}_{\pi}\left[\left|Y_{s}^{\mathrm{Q}}(\omega)-Y_{s}^{\mathrm{P}}(\bar{\omega})\right|^{\beta}\right] \mathrm{d} s \\
& +\mathbb{E}_{\pi}\left[\sup _{s \leq t}\left|\omega_{s}-\bar{\omega}_{s}\right|^{\beta}\right] .
\end{aligned}
$$

By Grönwall we have

$$
\mathbb{E}_{\pi}\left[\sup _{s \leq T}\left|Y_{s}^{\mathrm{Q}}(\omega)-Y_{s}^{\mathrm{P}}(\bar{\omega})\right|^{\beta}\right] \leq K^{\prime} \mathbb{E}_{\pi}\left[\sup _{s \leq T}\left|\omega_{s}-\bar{\omega}_{s}\right|^{\beta}\right],
$$

so taking infimum over such $\pi$ we conclude that

$$
\mathcal{W}_{\beta}(\Theta(\mathrm{Q}), \Theta(\mathrm{P})) \leq K^{\prime} \mathcal{W}_{\beta}(\mathrm{Q}, \mathrm{P})
$$

We now prove that $\Theta$ is injective. Let $P=\Theta(Q)=\Theta(\hat{Q})$. By definition we have Q-a.s.

$$
\begin{aligned}
\omega_{t} & =Y_{t}^{\mathrm{Q}}(\omega)-\int_{0}^{t}\left[\int b\left(s, Y_{s}^{\mathrm{Q}}(\omega), Y_{s}^{\mathrm{Q}}(\bar{\omega})\right) \mathrm{Q}(\mathrm{d} \bar{\omega})\right] \mathrm{d} s \\
& =Y_{t}^{\mathrm{Q}}(\omega)-\int_{0}^{t}\left[\int b\left(s, Y_{s}^{\mathrm{Q}}(\omega), \bar{\omega}\right) \mathrm{P}(\mathrm{d} \bar{\omega})\right] \mathrm{d} s,
\end{aligned}
$$

and the same holds for Q instead of Q. Denoting

$$
F(\omega):=\omega-\int_{0}^{\cdot}\left[\int b(s, \omega, \bar{\omega}) \mathrm{P}(\mathrm{d} \bar{\omega})\right] \mathrm{d} s,
$$

we therefore have

$$
\begin{aligned}
& \omega_{t}=F\left(Y^{\mathrm{Q}}\right)_{t}(\mathrm{Q}-a . s .), \\
& \omega_{t}=F\left(Y^{\hat{\mathrm{Q}}}\right)_{t}(\hat{\mathrm{Q}}-a . s .) .
\end{aligned}
$$

Hence $\mathrm{Q}=\Theta(\mathrm{Q}) \circ(F)^{-1}=\mathrm{P} \circ(F)^{-1}=\Theta(\hat{\mathrm{Q}}) \circ(F)^{-1}=\hat{\mathrm{Q}}$.

We can now provide the proof of Theorem 3.1:

Proof of Theorem 3.1 As we have observed, if $\left\{B^{i}: i \in \mathbb{N}\right\}$ is and iid sequence of $\mathrm{R}^{\mu^{\text {in }}}$-distributed processes, then $\frac{1}{N} \sum_{i=1}^{N} \delta_{B^{i}}$ satisfies a large deviations principle in $\mathcal{P}_{\beta}(\Omega)$ equipped with the $\mathcal{W}_{\beta}$ topology, with good rate function given by the relative entropy $\mathcal{H}\left(\cdot \mid \mathrm{R}^{\mu \text { in }}\right)$. By (48), and since $\Theta:\left(\mathcal{P}_{\beta}, \mathcal{W}_{\beta}\right) \rightarrow\left(\mathcal{P}_{\beta}, \mathcal{W}_{\beta}\right)$ is continuous, 
the contraction principle establishes that $\left\{\frac{1}{N} \sum_{i=1}^{N} \delta_{X^{i, N}}: N \in \mathbb{N}\right\}$ satisfies a large deviations principle in $\mathcal{P}_{\beta}(\Omega)$ equipped with the $\mathcal{W}_{\beta}$ topology. Since $\Theta$ is injective the good rate function is given by

$$
\tilde{\mathscr{I}}(\mathrm{P}):= \begin{cases}\mathcal{H}\left(\Theta^{-1}(\mathrm{P}) \mid \mathrm{R}^{\mu^{\text {in }}}\right) & \text { if } \mathrm{P} \in \operatorname{range}(\Theta) \\ +\infty & \text { otherwise. }\end{cases}
$$

In fact observe that if $\mathrm{P} \in \operatorname{range}(\Theta)$ and $\Theta^{-1}(\mathrm{P}) \ll \mathrm{R}^{\mu^{\text {in }}}$ then $^{7} \mathrm{P} \ll \mathrm{R}^{\mu^{\text {in }}}$, so

$$
\tilde{\mathscr{I}}(\mathrm{P}):= \begin{cases}\mathcal{H}\left(\Theta^{-1}(\mathrm{P}) \mid \mathrm{R}^{\mu^{\text {in }}}\right) & \text { if } \mathrm{P} \in \operatorname{range}(\Theta) \text { and } \mathrm{P} \ll \mathrm{R}^{\mu^{\text {in }}} \\ +\infty & \text { otherwise. }\end{cases}
$$

Now take $\mathrm{P} \in \operatorname{range}(\Theta)$ and call $\mathrm{Q}=\Theta^{-1}(\mathrm{P})$. It is immediate by the definition of $\Gamma(\cdot)$ that $\Gamma(\mathrm{P})=\mathrm{R}^{\mu^{\text {in }}} \circ\left(Y^{\mathrm{Q}}\right)^{-1}$. On the other hand observe that the filtration generated by $Y^{\mathrm{Q}}$ is equal to the canonical filtration: indeed $Y^{\mathrm{Q}}$ is adapted and conversely

$$
\omega_{t}=Y_{t}^{\mathrm{Q}}-\int_{0}^{t}\left[\int b\left(s, Y_{s}^{\mathrm{Q}}, \bar{\omega}\right) \mathrm{P}(\mathrm{d} \bar{\omega})\right] \mathrm{d} s=: h_{t}\left(Y^{Q}\right)
$$

so the canonical process is $Y^{\mathrm{Q}}$-adapted. From this

$$
\frac{\mathrm{d}\left(\mathrm{Q} \circ\left(Y^{\mathrm{Q}}\right)^{-1}\right)}{\mathrm{d}\left(\mathrm{R}^{\mu^{\text {in }}} \circ\left(Y^{\mathrm{Q}}\right)^{-1}\right)}=\mathbb{E}_{\mathrm{R}^{\mu^{\text {in }}}}\left[\frac{\mathrm{dQ}}{\mathrm{dR}^{\mu^{\text {in }}}} \mid \sigma\left(Y^{\mathrm{Q}}\right)\right]=\frac{\mathrm{dQ}}{\mathrm{dR}^{\mu^{\text {in }}}} \circ h .
$$

Hence

$$
\begin{aligned}
\mathcal{H}(\mathrm{P} \mid \Gamma(\mathrm{P})) & =\mathcal{H}\left(\mathrm{Q} \circ\left(Y^{\mathrm{Q}}\right)^{-1} \mid \mathrm{R}^{\mu^{\mathrm{in}}} \circ\left(Y^{\mathrm{Q}}\right)^{-1}\right)=\mathbb{E}_{\mathrm{Q} \circ\left(Y^{\mathrm{Q}}\right)^{-1}}\left[\log \frac{\mathrm{dQ}}{\mathrm{dR}^{\mu^{\text {in }}}} \circ h\right] \\
& =\mathcal{H}\left(\mathrm{Q} \mid \mathrm{R}^{\mu^{\text {in }}}\right)=\mathcal{H}\left(\Theta^{-1}(\mathrm{P}) \mid \mathrm{R}^{\mu^{\text {in }}}\right),
\end{aligned}
$$

and therefore

$$
\tilde{\mathscr{I}}(\mathrm{P})= \begin{cases}\mathcal{H}(\mathrm{P} \mid \Gamma(\mathrm{P})) & \text { if } \mathrm{P} \in \operatorname{range}(\Theta) \text { and } \mathrm{P} \ll \mathrm{R}^{\mu^{\text {in }}} \\ +\infty & \text { otherwise }\end{cases}
$$

The next step is to show that $\mathrm{P} \ll \mathrm{R}^{\mu^{\text {in }}}$ implies $\mathrm{P} \in \operatorname{range}(\Theta)$. In fact, denote by $\tau$ the adapted transformation

$$
\omega \mapsto \tau_{t}(\omega)=\omega_{t}-\int_{0}^{t} \int b(s, \omega, \bar{\omega}) \mathrm{P}(\mathrm{d} \bar{\omega}) \mathrm{d} s .
$$

${ }^{7}$ Let $\mathrm{P}=\Theta(\mathrm{Q})$ for $\mathrm{Q} \ll \mathrm{R}^{\mu}{ }^{\text {in }}$. The process $Y^{\mathrm{Q}}$ satisfies pointwise $\mathrm{d} Y_{t}^{\mathrm{Q}}=\mathrm{d} \omega_{t}+\bar{b}\left(t, Y^{\mathrm{Q}}\right) \mathrm{d} t$, where $\bar{b}(t, y)=\int b\left(t, y, Y^{\mathrm{Q}}(\bar{\omega})\right) \mathrm{Q}(\mathrm{d} \bar{\omega})$. We have $\mathrm{R}^{\mu^{\text {in }}} \circ\left(Y^{\mathrm{Q}}\right)^{-1} \ll \mathrm{R}^{\mu^{\text {in }}}$ since in fact their relative entropy is finite. Hence, if $\mathrm{R}^{\mu^{\text {in }}}(A)=0$ then $\mathrm{R}^{\mu^{\text {in }}}\left(\left(Y^{\mathrm{Q}}\right)^{-1}(A)\right)=0$, and so $\mathrm{Q} \ll \mathrm{R}^{\mu^{\text {in }}}$ implies $\mathrm{Q}\left(\left(Y^{\mathrm{Q}}\right)^{-1}(A)\right)=0$ therefore $\mathrm{P}(A)=0$ as desired. 
On the other hand call $X^{\mathrm{P}}$ the unique adapted pointwise solution to

$$
X_{t}^{\mathrm{P}}=\omega_{0}+\int_{0}^{t}\left[\int b\left(s, X^{\mathrm{P}}, \bar{\omega}\right) \mathrm{P}(\mathrm{d} \bar{\omega})\right] \mathrm{d} s+\omega_{t},
$$

which exists by Lemma 3.2 applied to the drift $\int b(\cdot, \cdot, \bar{\omega}) \mathrm{P}(\mathrm{d} \bar{\omega})$. As we recall in Lemma 5.4, $X^{\mathrm{P}}$ and $\tau$ are $\mathrm{P}$-a.s. inverses if $\mathrm{P} \ll \mathrm{R}^{\mu^{\text {in }}}$, since the above drift is Lipschitz. Now introduce $\mathrm{Q}:=\mathrm{P} \circ(\tau)^{-1}$, so that $\mathrm{Q} \circ\left(X^{\mathrm{P}}\right)^{-1}=\mathrm{P}$ and in particular

$$
X_{t}^{\mathrm{P}}=\omega_{0}+\int_{0}^{t}\left[\int b\left(s, X^{\mathrm{P}}, X^{\mathrm{P}}(\bar{\omega})\right) \mathrm{Q}(\mathrm{d} \bar{\omega})\right] \mathrm{d} s+\omega_{t}
$$

By Lemma 3.2 we have $\Theta(\mathrm{Q}):=Q \circ\left(Y^{\mathrm{Q}}\right)^{-1}=Q \circ\left(X^{\mathrm{P}}\right)^{-1}=\mathrm{P}$.

We have arrived at

$$
\tilde{\mathscr{I}}(\mathrm{P})= \begin{cases}\mathcal{H}(\mathrm{P} \mid \Gamma(\mathrm{P})) & \text { if } \mathrm{P} \ll \mathrm{R}^{\mu^{\text {in }}} \\ +\infty & \text { otherwise }\end{cases}
$$

To obtain the desired form (44) of the rate function it suffices to use Lemma 5.2 in the Appendix.

\subsection{McKean-Vlasov formulation and planning McKean-Vlasov FBSDE}

Proof of Lemma 1.1 and Proposition 1.1

Under $(\mathrm{H} 1)$ for any $\mathrm{P} \in \mathcal{P}_{1}(\Omega)$ the vector field

$$
[0, T] \times \mathbb{R}^{d} \ni(t, x) \mapsto-\nabla W * \mathrm{P}_{t}(x):=-\int_{\mathbb{R}^{d}} \nabla W(x-z) \mathrm{P}_{t}(\mathrm{~d} z),
$$

is very well-behaved. Precisely:

Lemma 3.5 Let $\mathrm{P} \in \mathcal{P}_{1}(\Omega)$ and grant $(\mathrm{H} 1)$. Then the time-dependent vector field $(t, x) \mapsto-\nabla W * \mathrm{P}_{t}(x)$ belongs $\mathcal{C}^{0,1}\left([0, T] \times \mathbb{R}^{d} ; \mathbb{R}^{d}\right)$ and is uniformly Lipschitz in the space variable.

Proof We begin by proving continuity. Fix $t, x$ and $\left(t_{n}, x_{n}\right) \rightarrow(t, x)$. The sequence $\nabla W\left(x_{n}-X_{t_{n}}\right)$ converges pointwise to $\nabla W\left(x-X_{t}\right)$, since $X$ is the (continuous) canonical process. By the fundamental theorem of calculus and (H1) we have $\left|\nabla W\left(x_{n}-X_{t_{n}}\right)\right| \leq C_{1}+C_{2} \sup _{s \in[0, T]}\left|X_{S}\right|$. Since $\mathrm{P} \in \mathcal{P}_{1}(\Omega)$, we may use dominated convergence to conclude $\mathbb{E}_{\mathrm{P}}\left[\nabla W\left(x_{n}-X_{t_{n}}\right)\right] \rightarrow \mathbb{E}_{\mathrm{P}}\left[\nabla W\left(x-X_{t}\right)\right]$. The space Lipschitzianity of $-\nabla W * \mathrm{P}_{t}$ follows from (H1). Space differentiability follows similarly from $(\mathrm{H} 1)$ and dominated convergence.

We will often make use of the next technical lemma, whose proof we defer to the appendix: 
Lemma 3.6 Let $\mu \in \mathcal{P}_{2}\left(\mathbb{R}^{d}\right)$ and $\bar{b}$ be of class $\mathcal{C}^{0,1}\left([0, T] \times \mathbb{R}^{d} ; \mathbb{R}^{d}\right)$ and such that

$$
\forall t \in[0, T], x, y \in \mathbb{R}^{d}|\bar{b}(t, x)-\bar{b}(t, y)| \leq C|x-y|
$$

for some $C<+\infty$. Define $\overline{\mathrm{R}}$ as the law of the $S D E$

$$
\mathrm{d} X_{t}=\bar{b}\left(t, X_{t}\right) \mathrm{d} t+\mathrm{d} B_{t}, \quad X_{0} \sim \mu
$$

and let $\mathrm{P} \in \mathcal{P}(\Omega)$ with $X_{0} \sim \mu$. The following are equivalent

(i) $\mathcal{H}(\mathrm{P} \mid \overline{\mathrm{R}})<+\infty$.

(ii) There exist a $\mathrm{P}$-a.s. defined adapted process $\left(\bar{\alpha}_{t}\right)_{t \in[0, T]}$ such that

$$
\mathbb{E}_{\mathrm{P}}\left[\int_{0}^{T}\left|\bar{\alpha}_{t}\right|^{2} \mathrm{~d} t\right]<+\infty
$$

and

$$
X_{t}-\int_{0}^{t}\left[\bar{b}\left(s, X_{s}\right)+\bar{\alpha}_{s}\right] \mathrm{d} s
$$

is a Brownian motion under $\mathrm{P}$.

Moreover, if (i), or equivalently (ii), holds, then we have

$$
\mathcal{H}(\mathrm{P} \mid \overline{\mathrm{R}})=\frac{1}{2} \mathbb{E}_{\mathrm{P}}\left[\int_{0}^{T}\left|\bar{\alpha}_{t}\right|^{2} \mathrm{~d} t\right]
$$

and

$$
\mathbb{E}_{\mathrm{P}}\left[\sup _{t \in[0, T]}\left|X_{t}\right|^{2}+\left|\bar{b}\left(t, X_{t}\right)^{2}\right|\right]<+\infty
$$

In particular, if (i), or equivalently (ii), holds we have that $\mathrm{P} \in \mathcal{P}_{2}(\Omega)$.

We turn to proving Lemma 1.1 stated in the introduction:

Proof of Lemma 1.1 Define the vector field $\bar{b}(t, z):=-\nabla W * \mathrm{P}_{t}(z)$. Lemma 3.5 grants that $\bar{b}$ fulfills the hypotheses of Lemma 3.6, giving the desired conclusions.

We can prove Proposition 1.1 of the introduction, concerning the existence of MFSBs. Recall the definition of $\Gamma(\mathrm{P})$ and (MFSP) from the introduction.

Proof of Proposition 1.1 Let $\mathrm{R}^{\mu^{\text {in }}}$ be the law of the Brownian motion started at $\mu^{\text {in }}$. (H2) grants that the classical Schrödinger problem (namely wrt. Brownian motion) is admissible. To see this, it suffices to verify that the coupling $\mu^{\text {in }} \otimes \mu^{\text {fin }}$ is admissible for the static version of the Schrödinger problem [36, Def 2.2] and then use the equivalence between the static and dynamic versions [36, Prop 2.3]. Therefore, there exist some 
$\mathrm{P} \in \mathcal{P}(\Omega)$ such that $\mathrm{P}_{0}=\mu^{\text {in }}$ and $\mathcal{H}\left(\mathrm{P} \mid \mathrm{R}^{\mu^{\text {in }}}\right)<+\infty$. Lemma 3.6 (or its specialization Lemma 5.1 in the appendix) yields that $\mathrm{P} \in \mathcal{P}_{1}(\Omega)$. On the other hand Lemma 5.2 in the appendix proves that for any $\mathrm{P} \in \mathcal{P}(\Omega) \mathcal{H}(\mathrm{P} \mid \Gamma(\mathrm{P}))<+\infty$ if and only if $\mathcal{H}\left(\mathrm{P} \mid \mathrm{R}^{\mu^{\text {in }}}\right)<$ $+\infty$. Thus (MFSP) is admissible as well. Now observe that $\mathrm{P} \mapsto \mathcal{H}(\mathrm{P} \mid \Gamma(\mathrm{P}))$ is lower semicontinuous in $\mathcal{P}_{\beta}(\Omega)$, since on the one hand the relative entropy is jointly lower semicontinuous in the weak topology, and on the other hand $\Gamma$ is readily seen to be continuous in $\mathcal{P}_{1}(\Omega)$. Recalling the definition of the operator $\Theta$ given in (47), to finish the proof we only need to justify that

$$
\theta_{M}:=\left\{\mathrm{P} \in \mathcal{P}_{1}(\Omega): \mathcal{H}\left(\Theta^{-1}(\mathrm{P}) \mid \mathrm{R}^{\mu^{\text {in }}}\right) \leq M, \mathrm{P}_{0}=\mu^{\text {in }}\right\}
$$

is relatively compact in $\mathcal{P}_{1}(\Omega)$ for each $M$, since the proof of Theorem 3.1 established $^{8}$ that $\mathcal{H}\left(\Theta^{-1}(\mathrm{P}) \mid \mathrm{R}^{\mu^{\text {in }}}\right)=\mathcal{H}(\mathrm{P} \mid \Gamma(\mathrm{P}))$ if $\mathrm{P} \ll \mathrm{R}^{\mu^{\text {in }}}$. Now remark that

$$
\theta_{M} \subset \Theta\left(\left\{\mathrm{Q}: \mathcal{H}\left(\mathrm{Q} \mid \mathrm{R}^{\mu^{\mathrm{in}}}\right) \leq M, \mathrm{Q}_{0}=\mu^{\mathrm{in}}\right\}\right) \subset \Theta\left(\left\{\mathrm{Q}: \mathcal{H}\left(\mathrm{Q} \mid \mathrm{R}^{\gamma}\right) \leq \bar{M}, \mathrm{Q}_{0}=\mu^{\mathrm{in}}\right\}\right)
$$

where $\gamma$ denotes the standard Gaussian, since by the decomposition of the entropy we have

$$
\mathcal{H}\left(\mathrm{P} \mid \mathrm{R}^{\gamma}\right)=\mathcal{H}\left(\mu^{\mathrm{in}} \mid \gamma\right)+\mathcal{H}\left(\mathrm{P} \mid \mathrm{R}^{\mu^{\text {in }}}\right)
$$

and by Assumption (H2)

$$
\begin{aligned}
\mathcal{H}\left(\mu^{\mathrm{in}} \mid \gamma\right) & =\int \log \mu^{\mathrm{in}}(x) \mu^{\mathrm{in}}(\mathrm{d} x)-\int \log (\gamma(x)) \mu^{\mathrm{in}}(\mathrm{d} x) \\
& =\int \log \mu^{\mathrm{in}}(x) \mu^{\mathrm{in}}(\mathrm{d} x)+c-\int \frac{|x|^{2}}{2} \mu^{\text {in }}(\mathrm{d} x)<\infty
\end{aligned}
$$

As $\Theta$ is per Lemma 3.4 Lipschitz in $\mathcal{P}_{1}(\Omega)$, it remains to prove that $\left\{\mathcal{H}\left(\mathrm{Q} \mid \mathrm{R}^{\gamma}\right) \leq \bar{M}\right\}$ is $\mathcal{W}_{1}$-compact. This can be easily done by hand, or by invoking Sanov Theorem in the $\mathcal{W}_{1}$-topology for independent particles distributed according to $\mathrm{R}^{\gamma}$ (see e.g. [56]), finishing the proof.

Proof of Theorem 1.3 We split the proof into two propositions, namely Propositions 3.1 and 3.2. We begin by addressing the issue of Markovianity of the minimizers. Recall the definition of $\mathrm{H}_{-1}\left(\left(\mu_{t}\right)_{t \in[0, T]}\right)$ given under 'frequently used notation.' We rely strongly on the work [14] by Cattiaux and Léonard for the proof of the following result:

\footnotetext{
8 This part of the proof did not use the existence of exponential moments for $\mu^{\text {in }}$. If we assume existence of exponential moments, then the compactness of $\theta_{M}$ follows from Theorem 3.1, since the rate function must be good.
} 
Proposition 3.1 Let $\mathrm{P}$ be optimal for (MFSP). Then there exists $\Psi \in \mathrm{H}_{-1}\left(\left(\mathrm{P}_{t}\right)_{t \in[0, T]}\right)$ such that

$$
(\mathrm{d} t \times \mathrm{dP} \text {-a.s. }) \quad \alpha_{t}^{\mathrm{P}}=\Psi_{t}\left(X_{t}\right),
$$

where $\left(\alpha_{t}^{\mathrm{P}}\right)_{t \in[0, T]}$ is given in Lemma 1.1 .

Proof If P be optimal for (MFSP), then it is also optimal for

$$
\inf \left\{\mathcal{H}(\mathrm{Q} \mid \Gamma(\mathrm{P})): \mathrm{Q} \in \mathcal{P}_{1}(\Omega), \mathrm{Q}_{t}=\mathrm{P}_{t} \text { for all } t \in[0, T]\right\}
$$

since $\Gamma(\mathrm{P})$ only depends on the marginals of $\mathrm{P}$. The above problem is an instance of [14], ie. its optimizer is a so-called critical Nelson process. However, the drift of the path-measure $\Gamma(\mathrm{P})$ may not fulfill the hypotheses in [14]. For this reason we need to make a slight detour. Let $\theta^{n} \in \mathcal{C}_{c}^{\infty}\left([0, T] \times \mathbb{R}^{d}\right)$ and $\mathrm{R}^{n}$ be defined as in Lemma 5.3 in the appendix, meaning that $\nabla \theta^{n}(\cdot)$ converges to $-\nabla W * \mathrm{P}_{t}(z)$ in $\mathrm{H}_{-1}\left(\left(\mathrm{P}_{t}\right)_{t \in[0, T]}\right)$ and that $\mathrm{R}^{n}$ is the law of

$$
\mathrm{d} Y_{t}=\nabla \theta_{t}^{n}\left(Y_{t}\right) \mathrm{d} t+\mathrm{d} B_{t}, \quad Y_{0} \sim \mu^{\text {in }} \in \mathcal{P}_{2}\left(\mathbb{R}^{d}\right) .
$$

For any $n$ consider the problem

$$
\min \left\{\mathcal{H}\left(\mathrm{Q} \mid \mathrm{R}^{n}\right): \mathrm{Q} \in \mathcal{P}_{1}(\Omega), \mathrm{Q}_{t}=\mathrm{P}_{t} \text { for all } t \in[0, T]\right\}
$$

Using [14, Lemma 3.1, Theorem 3.6] we obtain that for all $n$ the unique optimizer $\overline{\mathrm{P}}$ of (57) is the same for all $n$, and is such that there exists $\Phi \in \mathrm{H}_{-1}\left(\left(\mathrm{P}_{t}\right)_{t \in[0, T]}\right)$ such that

$$
X_{t}-\int_{0}^{t} \Phi_{s}\left(X_{s}\right) \mathrm{d} s
$$

is a Brownian motion under $\overline{\mathrm{P}}$. Lemma 3.5 grants that if we set $\bar{b}(t, z)=-\nabla W * \mathrm{P}_{t}(z)$ then the hypotheses of Lemma 3.6 are met. Since $\mathcal{H}(\mathrm{P} \mid \Gamma(\mathrm{P}))<+\infty$, we derive from (54) therein that

$$
\mathbb{E}_{\overline{\mathrm{P}}}\left[\int_{0}^{T}\left|\nabla W * \mathrm{P}_{t}\left(X_{t}\right)\right|^{2} \mathrm{~d} t\right]=\mathbb{E}_{\mathrm{P}}\left[\int_{0}^{T}\left|\nabla W * \mathrm{P}_{t}\left(X_{t}\right)\right|^{2} \mathrm{~d} t\right]<+\infty .
$$

Hence

$$
\mathbb{E}_{\overline{\mathrm{P}}}\left[\int_{0}^{T}\left|\Phi_{t}\left(X_{t}\right)+\nabla W * \mathrm{P}_{t}\left(X_{t}\right)\right|^{2} \mathrm{~d} t\right]<+\infty
$$

Using the implication $(i i) \Rightarrow(i)$ of Lemma 3.6 we finally obtain that $\mathcal{H}(\overline{\mathrm{P}} \mid \Gamma(\mathrm{P}))<$ $+\infty$ and therefore that we can use Lemma 5.3 for the choice $\mathrm{Q}=\overline{\mathrm{P}}$ therein. 
Now consider $\mathrm{Q}$ admissible for (56) and such that $\mathcal{H}(\mathrm{Q} \mid \Gamma(\mathrm{P}))<+\infty$. Using Lemma 5.3 twice we obtain

$$
\mathcal{H}(\overline{\mathrm{P}} \mid \Gamma(\mathrm{P}))=\liminf _{n \rightarrow+\infty} \mathcal{H}\left(\overline{\mathrm{P}} \mid \mathrm{R}^{n}\right) \leq \liminf _{n \rightarrow+\infty} \mathcal{H}\left(\mathrm{Q} \mid \mathrm{R}^{n}\right)=\mathcal{H}(\mathrm{Q} \mid \Gamma(\mathrm{P}))
$$

Thus $\overline{\mathrm{P}}$ is also an optimizer for (56). But then $\overline{\mathrm{P}}=\mathrm{P}$ since (56) can have at most one minimizer by strict convexity of the entropy and convexity of the admissible region. Combining (58) with (9) we get that $\int_{0}^{t}\left(-\nabla W * \mathrm{P}_{s}\left(X_{s}\right)+\alpha_{s}^{\mathrm{P}}-\Phi_{s}\left(X_{s}\right)\right) \mathrm{d} s$ is a continuous martingale with finite variation. But then it is constant $\mathrm{P}$-a.s. The conclusion follows setting $\Psi_{t}(z):=\Phi_{t}(z)+\nabla W * \mathrm{P}_{t}(z)$ and observing that $\nabla W * \mathrm{P} .(\cdot) \in$ $\mathrm{H}_{-1}\left(\left(\mathrm{P}_{t}\right)_{r \in[0, T]}\right)$.

Notice that the above proposition proves the first half of Theorem 1.3 from the introduction. We now establish the second half of this result:

Proposition 3.2 Assume that $\mathrm{P}$ is optimal for (MFSP). Then $\Psi_{t}\left(X_{t}\right)$ has a continuous version adapted to the $\mathrm{P}$-augmented canonical filtration, and the process $\left(M_{t}\right)_{t \in[0, T]}$ defined by

$$
M_{t}:=\Psi_{t}\left(X_{t}\right)-\int_{0}^{t} \tilde{\mathbb{E}}_{\tilde{\mathrm{P}}}\left[\nabla^{2} W\left(X_{s}-\tilde{X}_{s}\right) \cdot\left(\Psi_{s}\left(X_{s}\right)-\Psi_{s}\left(\tilde{X}_{S}\right)\right)\right] \mathrm{d} s
$$

is a continuous martingale under $\mathrm{P}$ on $\left[0, T\left[\right.\right.$ and satisfies $\mathbb{E}_{\mathrm{P}}\left[\int_{0}^{T}\left|M_{t}\right|^{2} \mathrm{~d} t\right]<+\infty$.

To carry out the proof, we will use a well-known characterization of martingales (see e.g. [23]) which is as follows: an adapted process $\left(M_{t}\right)_{t \in[0, T]}$ such that $\mathbb{E}_{\mathrm{P}}\left[\int_{0}^{T}\left|M_{t}\right|^{2} \mathrm{~d} t\right]<+\infty$ is a martingale in $[0, T[$ under $\mathrm{P}$ if and only if

$$
\mathbb{E}_{\mathrm{P}}\left[\int_{0}^{T} M_{t} h_{t} \mathrm{~d} t\right]=0
$$

for all adapted processes $\left(h_{t}\right)_{t \in[0, T]}$ such that

$$
\mathbb{E}_{\mathrm{P}}\left[\int_{0}^{T}\left|h_{t}\right|^{2} \mathrm{~d} t\right]<+\infty, \quad \text { and } \int_{0}^{T} h_{t} \mathrm{~d} t=0 \quad \mathrm{P}-\text { a.s. }
$$

Proof Define $\left(M_{t}\right)_{t \in[0, T]}$ via (60). Using (H1), (8) and (54) we get that $\mathbb{E}_{\mathrm{P}}\left[\int_{0}^{T}\left|M_{t}\right|^{2} \mathrm{~d} t\right]$ $<+\infty$. Therefore, using the characterization of martingales [23, pp. 148-149] in order to show that $M_{t}$ is a martingale on [0,T [ we need to show (61) for all adapted processes $\left(h_{t}\right)_{t \in[0, T]}$ satisfying (62). By a standard density argument, one can show that it suffices to obtain (61) under the additional assumption that $\left(h_{t}\right)_{t \in[0, T]}$ is bounded and Lipschitz, i.e.

$$
\begin{aligned}
\forall t & \in[0, T], \omega, \bar{\omega} \in \Omega, \quad \sup _{s \in[0, t]}\left|h_{s}(\omega)-h_{s}(\bar{\omega})\right| \\
& \leq C \sup _{s \in[0, t]}\left|\omega_{s}-\bar{\omega}_{s}\right|, \quad \sup _{t \in[0, T]}\left|h_{t}(\omega)\right| \leq C,
\end{aligned}
$$


for some $C>0$. Consider now a process $\left(h_{t}\right)_{t \in[0, T]}$ satisfying (62) and (63) and for $\varepsilon>0$ define the shift transformation

$$
\tau^{\varepsilon}: \Omega \longrightarrow \Omega, \quad \tau_{t}^{\varepsilon}(\omega)=\omega_{t}+\varepsilon \int_{0}^{t} h_{s}(\omega) \mathrm{d} s
$$

Under the current assumptions, $\tau^{\varepsilon}$ admits an adapted inverse $Y^{\varepsilon}$, i.e. there exists an adapted process $\left(Y_{t}^{\varepsilon}\right)_{t \in[0, T]}$ such that

$$
\mathrm{P}-\text { a.s. } \quad \tau_{t}^{\varepsilon}\left(Y^{\varepsilon}(\omega)\right)=Y_{t}^{\varepsilon}\left(\tau^{\varepsilon}(\omega)\right)=\omega_{t} \quad \forall t \in[0, T] .
$$

Indeed, since $\mathcal{H}(\mathrm{P} \mid \Gamma(\mathrm{P}))<+\infty$, Lemma 5.2 in the appendix yields that $\mathrm{P} \ll \mathrm{R}^{\mu^{\text {in }}}$; this entitles us to apply Lemma 5.4 in the same section, providing the existence of the inverse $Y^{\varepsilon}$.

If we set $\mathrm{P}^{\varepsilon}=\mathrm{P} \circ\left(\tau^{\varepsilon}\right)^{-1}$ we have that $\mathrm{P}^{\varepsilon} \in \mathcal{P}_{1}(\Omega)$ is admissible for (MFSP), thanks to (62). Moreover, Lemma 1.1 and (65) imply that

$$
X_{t}-\int_{0}^{t}\left(\varepsilon h_{s}\left(Y^{\varepsilon}\right)+\Psi_{s}\left(Y_{s}^{\varepsilon}\right)-\nabla W * \mathrm{P}_{s}\left(Y_{s}^{\varepsilon}\right)\right) \mathrm{d} s
$$

is a Brownian motion under $\mathrm{P}^{\varepsilon}$. Combining (8), (63) and (H1) we get that

$$
\frac{1}{2} \mathbb{E}_{\mathrm{P}^{\varepsilon}}\left[\int_{0}^{T}\left|\Psi_{t}\left(Y_{t}^{\varepsilon}\right)+\varepsilon h_{t}\left(Y^{\varepsilon}\right)-\nabla W * \mathrm{P}_{t}\left(Y_{t}^{\varepsilon}\right)+\nabla W * \mathrm{P}_{t}^{\varepsilon}\left(X_{t}\right)\right|^{2} \mathrm{~d} t\right]<+\infty .
$$

Lemma 3.5 grants that $\bar{b}(t, x)=-\nabla W * \mathrm{P}_{t}^{\varepsilon}(x)$ fulfills the hypothesis of Lemma 3.6 and (66) allows to use the implication $(i i) \Rightarrow(i)$ which yields that $\mathcal{H}\left(\mathrm{P}^{\varepsilon} \mid \Gamma\left(\mathrm{P}^{\varepsilon}\right)\right)$ is finite and equals the left hand side of (66). Using the definition of $\mathrm{P}^{\varepsilon}$, we can rewrite $\mathcal{H}\left(\mathrm{P}^{\varepsilon} \mid \Gamma\left(\mathrm{P}^{\varepsilon}\right)\right)$ as

$$
\frac{1}{2} \mathbb{E}_{\mathrm{P}}\left[\int_{0}^{T}\left|\varepsilon h_{t}+\Psi_{t}\left(X_{t}\right)+\nabla W * \mathrm{P}_{t}^{\varepsilon}\left(\tau_{t}^{\varepsilon}\right)-\nabla W * \mathrm{P}_{t}\left(X_{t}\right)\right|^{2} \mathrm{~d} s\right] .
$$

Imposing optimality of $\mathrm{P}$ and letting $\varepsilon$ to zero, using Taylor's expansion

$$
\begin{aligned}
0 & \leq \liminf _{\varepsilon \rightarrow 0} \frac{\mathcal{H}\left(\mathrm{P}^{\varepsilon} \mid \Gamma\left(\mathrm{P}^{\varepsilon}\right)\right)-\mathcal{H}(\mathrm{P} \mid \Gamma(\mathrm{P}))}{\varepsilon} \\
& =\mathbb{E}_{\mathrm{P}}\left[\int_{0}^{T} \Psi_{t}\left(X_{t}\right) \cdot\left(h_{t}+\tilde{\mathbb{E}}_{\tilde{\mathrm{P}}}\left[\nabla^{2} W\left(X_{t}-\tilde{X}_{t}\right) \cdot \int_{0}^{t} h_{s}-\tilde{h}_{s} \mathrm{~d} s\right]\right) \mathrm{d} t\right] .
\end{aligned}
$$

In the above equation, $\left(\tilde{X}_{t}, \tilde{h}_{t}\right)_{t \in[0, T]}$ is an independent copy of $\left(X_{t}, h_{t}\right)_{t \in[0, T]}$ defined on some probability space $(\tilde{\Omega}, \tilde{\mathfrak{F}}, \tilde{\mathrm{P}})$ and $\tilde{\mathbb{E}}_{\tilde{\mathrm{P}}}$ denotes the expectation on $(\tilde{\Omega}, \tilde{\mathfrak{F}}, \tilde{\mathrm{P}})$. Moreover, the exchange of limit and expectation is justified by (49), (8) and the dominated convergence theorem. Using the symmetry of $W$, and taking $\pm h$, we can rewrite the latter condition as 


$$
\begin{aligned}
0= & \mathbb{E}_{\mathrm{P}}\left[\int_{0}^{T} \Psi_{t}\left(X_{t}\right) \cdot h_{t} \mathrm{~d} t\right] \\
& +\mathbb{E}_{\mathrm{P}}\left[\int_{0}^{T} \tilde{\mathbb{E}}_{\tilde{\mathrm{P}}}\left[\left(\Psi_{t}\left(X_{t}\right)-\Psi_{t}\left(\tilde{X}_{t}\right)\right) \cdot \nabla^{2} W\left(X_{t}-\tilde{X}_{t}\right)\right] \cdot \int_{0}^{t} h_{s} \mathrm{~d} s \mathrm{~d} t\right] .
\end{aligned}
$$

By integration by parts and the boundary condition (62), we arrive at

$0=\mathbb{E}_{\mathrm{P}}\left[\int_{0}^{T}\left(\Psi_{t}\left(X_{t}\right)-\int_{0}^{t} \tilde{\mathbb{E}}_{\tilde{\mathrm{P}}}\left[\left(\Psi_{s}\left(X_{S}\right)-\Psi_{s}\left(\tilde{X}_{S}\right)\right) \cdot \nabla^{2} W\left(X_{S}-\tilde{X}_{S}\right)\right] \mathrm{d} s\right) \cdot h_{t} \mathrm{~d} t\right]$,

proving the desired martingale property. By [45, Theorem IV.36.5] we know that a martingale in an augmented Brownian filtration admits a continuous version. Using again Lemma 5.2 we have that $\mathrm{P} \ll \mathrm{R}^{\mu}$, and we so obtain a continuous version of our martingale (60), and a fortiori of $\Psi_{t}\left(X_{t}\right)$.

\subsection{Benamou-Brenier formulation}

We finally turn to the Benamou-Brenier formulation. Recall that $\mathscr{C}_{T}\left(\mu^{\mathrm{in}}, \mu^{\text {fin }}\right)$ denotes the optimal value of the mean field Schrödinger problem. We define the set $\mathcal{A}$ as the collection of all absolutely continuous curves $\left(\mu_{t}\right)_{t \in[0, T]} \subset \mathcal{P}_{2}\left(\mathbb{R}^{d}\right)$ (see Sect. 4.2) such that

$$
\begin{gathered}
(t, z) \mapsto \nabla \log \mu_{t}(z) \in L^{2}\left(\mathrm{~d} \mu_{t} \mathrm{~d} t\right), \\
(t, z) \mapsto \nabla W * \mu_{t}(z) \in L^{2}\left(\mathrm{~d} \mu_{t} \mathrm{~d} t\right) .
\end{gathered}
$$

Recall from the introduction the problem

$$
\begin{aligned}
& \mathscr{C}_{T}^{B B}\left(\mu^{\mathrm{in}}, \mu^{\mathrm{fin}}\right) \\
& :=\inf _{\substack{\left(\mu_{t}\right)_{t \in[0, T]} \in \mathcal{A}, \partial_{t} \mu_{t}+\nabla \cdot\left(w_{t} \mu_{t}\right)=0}} \frac{1}{2} \iint \mid w_{t}(z)+\frac{1}{2} \nabla \log \mu_{t}(z) \\
& \quad+\left.\nabla W * \mu_{t}(z)\right|^{2} \mu_{t}(\mathrm{~d} z) \mathrm{d} t
\end{aligned}
$$

In (67), solutions to the continuity equation $\partial_{t} \mu_{t}+\nabla \cdot\left(w_{t} \mu_{t}\right)=0$ are meant in the weak sense.

Proof of Theorem 1.2 We first show that $\mathscr{C}_{T}\left(\mu^{\mathrm{in}}, \mu^{\mathrm{fin}}\right) \geq \mathscr{C}_{T}^{B B}\left(\mu^{\mathrm{in}}, \mu^{\mathrm{fin}}\right)$. To this end, we may assume that the 1.h.s. if finite and denote $\mathrm{P}$ an optimizer. As established in Theorem 1.3, the drift of $X$ under $\mathrm{P}$ is equal to

$$
\int_{0}^{t} \Psi_{s}\left(X_{s}\right)-\nabla W * \mathrm{P}_{s}\left(X_{s}\right) \mathrm{d} s
$$


where $\Psi \in \mathrm{H}_{-1}\left(\left(\mathrm{P}_{t}\right)_{t \in[0, T]}\right)$ and

$$
\mathscr{C}_{T}\left(\mu^{\mathrm{in}}, \mu^{\mathrm{fin}}\right)=\frac{1}{2} \iint\left|\Psi_{t}(z)\right|^{2} \mathrm{P}_{t}(\mathrm{~d} z) \mathrm{d} t .
$$

As we will see in Lemma 4.4 and Remark 4.1, the flow of marginals $\left(\mathrm{P}_{t}\right)_{t \in[0, T]}$ is absolutely continuous and its tangent velocity field $v$ is given by

$$
v_{t}(z):=-\nabla W * \mathrm{P}_{t}(z)+\Psi_{t}(z)-\frac{1}{2} \nabla \log \mathrm{P}_{t} .
$$

Hence

$$
\mathscr{C}_{T}\left(\mu^{\mathrm{in}}, \mu^{\mathrm{fin}}\right)=\frac{1}{2} \iint\left|v_{t}(z)+\frac{1}{2} \nabla \log \mathrm{P}_{t}+\nabla W * \mathrm{P}_{t}(z)\right|^{2} \mathrm{P}_{t}(\mathrm{~d} z) \mathrm{d} t .
$$

We conclude the desired inequality by noticing that $\nabla \log \mathrm{P}_{t} \in L^{2}\left(\mathrm{dP}_{t} \mathrm{~d} t\right)$ and $\nabla W *$ $\mathrm{P}_{t} \in L^{2}\left(\mathrm{dP}_{t} \mathrm{~d} t\right)$. To wit, the first statement follows from [25, Thm 3.10] combined with Lemma 5.2 in our appendix, and the second from (54) used with $\bar{b}=-\nabla W * \mathrm{P}_{t}(z)$. We now establish $\mathscr{C}_{T}\left(\mu^{\mathrm{in}}, \mu^{\mathrm{fin}}\right) \leq \mathscr{C}_{T}^{B B}\left(\mu^{\mathrm{in}}, \mu^{\mathrm{fin}}\right)$, so we may assume that $\left(\mu_{t}\right)_{t \in[0, T]}$ is feasible for the r.h.s. and leads to a finite value. Denote by $\tilde{v}$ its tangent velocity field. We define $\Phi_{t}(z):=\tilde{v}_{t}(z)+\frac{1}{2} \nabla \log \mu_{t}(z)$, so from the continuity equation for $\left(\mu_{t}\right)_{t \in[0, T]}$ we deduce the following equation in the distributional sense

$$
\partial_{t} \mu_{t}+\nabla \cdot\left(\mu_{t} \Phi_{t}\right)-\frac{1}{2} \Delta \mu_{t}=0
$$

Observing that $\Phi \in \mathrm{H}_{-1}\left(\left(\mu_{t}\right)_{t \in[0, T]}\right)$, we may apply the equivalence "(a) iff (c)" in [14, Theorem 3.4]. ${ }^{9}$ We thus obtain a measure $\mathrm{P}$ whose marginals are exactly $\left(\mu_{t}\right)_{t \in[0, T]}$, and by the uniqueness statement in [14, Theorem 3.4] we also know that the drift of $X$ under $\mathrm{P}$ is precisely $\Phi_{S}\left(X_{S}\right)$. Hence

$$
\begin{aligned}
\frac{1}{2} \iint\left|\tilde{v}_{t}(z)+\frac{1}{2} \nabla \log \mu_{t}(z)+\nabla W * \mu_{t}(z)\right|^{2} \mu_{t}(\mathrm{~d} z) \mathrm{d} t \\
\quad=\frac{1}{2} \iint\left|\Phi_{t}(z)+\nabla W * \mu_{t}(z)\right|^{2} \mu_{t}(\mathrm{~d} z) \mathrm{d} t \\
\quad=\frac{1}{2} \mathbb{E}_{\mathrm{P}}\left[\int_{0}^{T}\left|\Phi\left(X_{t}\right)+\nabla W * \mathrm{P}_{t}\left(X_{t}\right)\right|^{2} \mathrm{~d} t\right] \\
\geq \mathscr{C}_{T}\left(\mu^{\text {in }}, \mu^{\mathrm{fin}}\right),
\end{aligned}
$$

where the inequality follows from the equivalent expression of $\mathscr{C}_{T}\left(\mu^{\mathrm{in}}, \mu^{\mathrm{fin}}\right)$ given in (10).

\footnotetext{
9 That is for the construction of a Nelson process with marginals $\left(\mu_{t}\right)_{t \in[0, T]}$, with respect to the reference measure given by Wiener started at $\mu^{\text {in }}$. 
We have proven $\mathscr{C}_{T}\left(\mu^{\mathrm{in}}, \mu^{\mathrm{fin}}\right)=\mathscr{C}_{T}^{B B}\left(\mu^{\mathrm{in}}, \mu^{\text {fin }}\right)$, and the other statements follow from the previous arguments.

\subsection{Schrödinger potentials and mean field PDE system: proofs}

We start with an observation concerning the link between (15) and (16):

Remark 3.1 It is worth stressing that the link between (15) and (16) can be established if the FBSDE solution $Y_{t}$ is a gradient vector field depending only on $t$ and $X_{t}$. We have gathered preliminary evidence that (15) admits non Markov solutions even in the simple case when $W=0$. More precisely, we expect that all processes in the reciprocal class of Brownian motion (meaning that they share the same bridges, but see [37] for details) fulfilling the marginal constraints of (1.1) are solutions to (15). This is in contrast with what is expected for standard FBSDEs [10, Lemma 3.5] whose boundary conditions are not of planning type.

We now provide the belated proofs:

Proof of Corollary 1.1 We know by Theorem 1.1 that $\Psi$ belongs to $\mathrm{H}\left(\left(\mu_{t}\right)\right)_{t \in[0, T]}$. The regularity hypothesis imposed on $\Psi_{t}(x)$ and $\mu_{t}(x)$ allow us to conclude that $\Psi$ is a true gradient, i.e. there exist $\psi$ such that $\Psi_{t}(x)=\nabla \psi_{t}(x)$ for all $(t, x) \in[0, T] \times \mathbb{R}^{d}$. Lemma 1.1 together with Theorem 1.3 yield that $\mu_{t}$ is a weak solution of the Fokker Planck equation in (17). Because of the regularity assumptions we made on $\Psi$ and $\mu$, we can conclude that $\mu_{t}$ is indeed a classical solution. For the same reasons, we can turn the martingale condition (1.3) into the system of PDEs

$$
\begin{aligned}
\forall i= & 1, \ldots, d \quad \partial_{t} \partial_{x_{i}} \psi_{t}(x)+\mathcal{L}\left(\partial_{x_{i}} \psi_{t}(x)\right) \\
& -\int_{\mathbb{R}^{d}} \partial_{x_{i}}\left((\nabla W(x-\tilde{x})) \cdot(\nabla \psi(x)-\nabla \psi(\tilde{x})) \mu_{t}(\mathrm{~d} \tilde{x})=0\right.
\end{aligned}
$$

where $\mathcal{L}$ is the generator $\frac{1}{2} \Delta+\left(\nabla\left(-W * \mu_{t}+\psi_{t}\right)\right) \cdot \nabla$. After some tedious but standard calculations we can rewrite the above as

$$
\begin{aligned}
& \partial_{x_{i}}\left(\partial_{t} \psi_{t}(x)+\frac{1}{2} \Delta \psi_{t}(x)+\frac{1}{2}\left|\nabla \psi_{t}(x)\right|^{2}\right. \\
& \left.\quad+\int_{\mathbb{R}^{d}} \nabla W(x-\tilde{x}) \cdot\left(\nabla \psi_{t}(x)-\nabla \psi_{t}(\tilde{x}) \mu_{t}(\mathrm{~d} \tilde{x})\right)\right)=0
\end{aligned}
$$

Since $\psi$ is defined up to the addition of a function that depends on time only, the conclusion follows.

Corollary 1.2 can be proven with a direct calculation using the definition of $\varphi_{t}$ and (17). 


\section{Convergence to equilibrium and functional inequalities: proofs}

In this part we complement the discussion undertaken in Sect. 1.4 and provide proofs for the results stated therein. This section is organized as follows:

- Sections 4.1, 4.2, 4.3 are devoted to stating and proving some preparatory results that we shall use at different times in the proofs of the main results.

- In Section 4.4 we prove Theorem 1.6, and we state and prove Theorem 4.1 together with its corollaries: the Talagrand (Corollary 1.3) and the HWI (Corollary 1.4) inequalities.

- Finally, in Sect. 4.5 we prove Theorems 1.4 and 1.5.

In all the lemmas and theorems in this subsection we always assume (H1)-(H2) to hold, and throughout $\mathrm{P}, \alpha^{\mathrm{P}}, \Psi, M$ are as given in Theorem 1.3. We refer to Sects. 1.2 and 1.4 for any unexplained notation.

\subsection{Exponential upper bound for the corrector}

Recall that we called $\Psi$ the corrector. The goal of this part is to quantify the size of the corrector, as stated in Lemma 4.3 below. Before doing this we prove two preliminary lemmas. As usual, we denote by $\langle\cdot\rangle$ the quadratic variation of a semimartingale.

Lemma 4.1 We have

$$
\forall t \in\left[0, T\left[, \quad \mathbb{E}_{\mathrm{P}}\left[\left|M_{t}\right|^{2}\right]=\mathbb{E}_{\mathrm{P}}\left[\langle M\rangle_{t}\right]<+\infty .\right.\right.
$$

Moreover the function $t \mapsto \mathbb{E}\left[\langle M\rangle_{t}\right]$ is continuous on $[0, T[$ and

$$
\forall t \in\left[0, T\left[, \sup _{s \in[0, t]} \mathbb{E}_{\mathrm{P}}\left[\left|\Psi_{s}\left(X_{s}\right)\right|^{2}\right]<+\infty\right.\right.
$$

Proof We have shown at Theorem 3.2 that $\mathbb{E}_{\mathrm{P}}\left[\int_{0}^{T}\left|M_{t}\right|^{2} \mathrm{~d} t\right]<+\infty$ which gives that $\mathbb{E}_{\mathrm{P}}\left[\left|M_{t}\right|^{2}\right]<+\infty$ for almost every $t \in\left[0, T\left[\right.\right.$. But since $\mathbb{E}_{\mathrm{P}}\left[\left|M_{t}\right|^{2}\right]$ is an increasing function of $t$, we get $\mathbb{E}_{\mathrm{P}}\left[\left|M_{t}\right|^{2}\right]<+\infty$ for all $t \in[0, T$. To complete the proof of (68) it suffices to observe that by definition of quadratic variation and since $M_{t}$ is an $L^{2}$-martingale on $\left[0, T\right.$, we have $\mathbb{E}_{\mathrm{P}}\left[\left|M_{t}\right|^{2}\right]=\mathbb{E}_{\mathrm{P}}\left[\left\langle M_{t}\right\rangle\right]$. To prove the continuity of $t \mapsto \mathbb{E}_{\mathrm{P}}\left[\langle M\rangle_{t}\right]$ we start by observing that since $M_{t}$ is a continuous martingale, then $\langle M\rangle_{t}$ has continuous and increasing paths. Thus, we obtain by monotone convergence that $\mathbb{E}_{\mathrm{P}}\left[\langle M\rangle_{t+h}\right] \rightarrow \mathbb{E}_{\mathrm{P}}\left[\langle M\rangle_{t}\right]$ as $h \downarrow 0$, which gives the desired result. The proof of (69) follows from (60), the bounded Hessian of $W$ (see(H1)) and the first part of Theorem 1.3.

Lemma 4.2 The function $t \mapsto \mathbb{E}_{\mathrm{P}}\left[X_{t}\right]$ is linear, the function $t \mapsto \mathbb{E}_{\mathrm{P}}\left[\Psi_{t}\left(X_{t}\right)\right]$ is constant, and

$$
\forall t \in\left[0, T\left[, \quad \mathbb{E}_{\mathrm{P}}\left[X_{t}\right]=\mathbb{E}_{\mathrm{P}}\left[X_{0}\right]+\mathbb{E}_{\mathrm{P}}\left[\Psi_{0}\left(X_{0}\right)\right] t\right.\right.
$$


Proof Using the symmetry of $W$ and the martingale property (60) it is easily derived that $\mathbb{E}_{\mathrm{P}}\left[\Psi_{t}\left(X_{t}\right)\right]$ is constant as a function of $t$. Therefore we get for all $t \in[0, T]$

$$
\mathbb{E}_{\mathrm{P}}\left[X_{t}\right]=\mathbb{E}_{\mathrm{P}}\left[X_{0}\right]-\int_{0}^{t} \mathbb{E}_{\mathrm{P}}\left[\nabla W * \mathrm{P}_{s}\left(X_{s}\right)\right] \mathrm{d} s+\mathbb{E}_{\mathrm{P}}\left[\Psi_{0}\left(X_{0}\right)\right] t
$$

Using again the symmetry of $W$ we get that $\int_{0}^{t} \mathbb{E}_{\mathrm{P}}\left[\nabla W * \mathrm{P}_{S}\left(X_{S}\right)\right] \mathrm{d} s=0$, from which the conclusion follows.

We can now provide some key estimates on the corrector:

Lemma 4.3 Assume (H1)-(H4). If $\mathrm{P}$ is an optimizer for (MFSP) and $\Psi$ the associated corrector, then for any $t \in(0, T)$ we have

$$
\frac{1}{2} \mathbb{E}_{\mathrm{P}}\left[\int_{0}^{t}\left|\Psi_{S}\left(X_{S}\right)\right|^{2} \mathrm{~d} s\right] \leq \frac{\exp (2 \kappa t)-1}{\exp (2 \kappa T)-1} \mathscr{C}_{T}\left(\mu^{\text {in }}, \mu^{\mathrm{fin}}\right),
$$

and

$$
\frac{1}{2} \mathbb{E}_{\mathrm{P}}\left[\left|\Psi_{t}\left(X_{t}\right)\right|^{2}\right] \leq \frac{2 \kappa \mathscr{C}_{T}\left(\mu^{\mathrm{in}}, \mu^{\mathrm{fin}}\right)}{\exp (2 \kappa(T-t))-1}
$$

Proof Consider the function $t \mapsto \varphi(t)$ defined by

$$
\varphi(t)=\frac{1}{2} \mathbb{E}_{\mathrm{P}}\left[\int_{0}^{t}\left|\Psi_{s}\left(X_{s}\right)\right|^{2} \mathrm{~d} s\right] .
$$

Fubini's theorem allows to interchange the time integral and the expectation to get that $\varphi$ is an absolutely continuous function with derivative

$$
\varphi^{\prime}(t)=\mathbb{E}_{\mathrm{P}}\left[\left|\Psi_{t}\left(X_{t}\right)\right|^{2}\right]
$$

From Itô's formula and Theorem 1.3 we get that for all $t \in[0, T[$

$$
\begin{aligned}
\left|\Psi_{t}\left(X_{t}\right)\right|^{2}-\left|\Psi_{0}\left(X_{0}\right)\right|^{2}= & 2 \int_{0}^{t} \Psi_{r}\left(X_{r}\right) \cdot \mathrm{d} M_{r} \\
& +2 \int_{0}^{t} \Psi_{r}\left(X_{r}\right) \cdot \tilde{\mathbb{E}}_{\tilde{\mathrm{P}}}\left[\nabla^{2} W\left(X_{r}-\tilde{X}_{r}\right)\right. \\
& \left.\left(\Psi_{r}\left(X_{r}\right)-\Psi_{r}\left(\tilde{X}_{r}\right)\right)\right] \mathrm{d} r+\langle M\rangle_{t} .
\end{aligned}
$$

We observe that the fact that $M_{t}$ is a martingale together with (69) and (68) make sure that $\mathbb{E}_{\mathrm{P}}\left[\int_{0}^{t} \Psi_{r}\left(X_{r}\right) \cdot \mathrm{d} M_{r}\right]=0$. Thus, taking expectation on both sides of the above 
equation yields

$$
\begin{aligned}
\varphi^{\prime}(t)-\varphi^{\prime}(0)= & \mathbb{E}_{\mathrm{P}}\left[2 \int_{0}^{t} \Psi_{r}\left(X_{r}\right) \cdot \tilde{\mathbb{E}}_{\tilde{\mathrm{P}}}\left[\nabla^{2} W\left(X_{r}-\tilde{X}_{r}\right) \cdot\left(\Psi_{r}\left(X_{r}\right)-\Psi_{r}\left(\tilde{X}_{r}\right)\right)\right] \mathrm{d} r\right] \\
& +\mathbb{E}_{\mathrm{P}}\left[\langle M\rangle_{t}\right] .
\end{aligned}
$$

Because of (69) we can use Fubini's Theorem and write

$$
\begin{aligned}
\mathbb{E}_{\mathrm{P}} & {\left[2 \int_{0}^{t} \Psi_{r}\left(X_{r}\right) \cdot \tilde{\mathbb{E}}_{\tilde{\mathrm{P}}}\left[\nabla^{2} W\left(X_{r}-\tilde{X}_{r}\right) \cdot\left(\Psi_{r}\left(X_{r}\right)-\Psi_{r}\left(\tilde{X}_{r}\right)\right)\right] \mathrm{d} r\right] } \\
& =2 \int_{0}^{t} \mathbb{E}_{\mathrm{P}}\left[\Psi_{r}\left(X_{r}\right) \cdot \tilde{\mathbb{E}}_{\tilde{\mathrm{P}}}\left[\nabla^{2} W\left(X_{r}-\tilde{X}_{r}\right) \cdot\left(\Psi_{r}\left(X_{r}\right)-\Psi_{r}\left(\tilde{X}_{r}\right)\right)\right]\right] \mathrm{d} r \\
& =\int_{0}^{t} \mathbb{E}_{\mathrm{P} \otimes \tilde{\mathrm{P}}}\left[\left(\Psi_{r}\left(X_{r}\right)-\Psi_{r}\left(\tilde{X}_{r}\right)\right) \cdot \nabla^{2} W\left(X_{r}-\tilde{X}_{r}\right) \cdot\left(\Psi_{r}\left(X_{r}\right)-\Psi_{r}\left(\tilde{X}_{r}\right)\right)\right] \mathrm{d} r
\end{aligned}
$$

where we used the symmetry of $W$ to obtain the last expression. Plugging it back in (73) and using that $t \mapsto \varphi^{\prime}(t)$ is

- continuous on $\left[0, T\left[\right.\right.$ because so are (74) and $\mathbb{E}\left[\langle M\rangle_{t}\right]$ (cf. Lemma 4.1),

- increasing on $[0, T$ [ since $W$ is convex and the quadratic variation is an increasing process,

we conclude that $t \mapsto \varphi^{\prime}(t)$ is absolutely continuous on the same interval. Moreover, using the $\kappa$-convexity of $W$ and again the fact that the quadratic variation is an increasing process we get

$$
\varphi^{\prime \prime}(t) \geq 2 \kappa \mathbb{E}_{\mathrm{P}}\left[\left|\Psi_{t}\left(X_{t}\right)\right|^{2}\right]=2 \kappa \varphi^{\prime}(t)
$$

where to establish the last inequality we used that the hypothesis on $\mu^{\text {in }}$ and $\mu^{\text {fin }}$ together with Lemma 4.2 imply $\mathbb{E}_{\mathrm{P}}\left[\Psi_{t}\left(X_{t}\right)\right]=0$. The bound (70) follows by integrating the differential inequality (75) as done for instance in Lemma 5.5 in the Appendix, and observing that $\frac{1}{2} \mathbb{E}_{\mathrm{P}}\left[\int_{0}^{T}\left|\Psi_{r}\left(X_{r}\right)\right|^{2} \mathrm{~d} r\right]=\mathcal{H}(\mathrm{P} \mid \Gamma(\mathrm{P}))$. To prove (71), we begin by observing that (75) also yields that

$$
\forall s \in[t, T], \quad \mathbb{E}_{\mathrm{P}}\left[\left|\Psi_{s}\left(X_{s}\right)\right|^{2}\right] \geq \exp (2 \kappa(s-t)) \mathbb{E}_{\mathrm{P}}\left[\left|\Psi_{t}\left(X_{t}\right)\right|^{2}\right] .
$$

Next, by definition of entropic cost we get the trivial bound

$$
\mathscr{C}_{T}\left(\mu^{\mathrm{in}}, \mu^{\mathrm{fin}}\right)=\frac{1}{2} \mathbb{E}_{\mathrm{P}}\left[\int_{0}^{T}\left|\Psi_{S}\left(X_{S}\right)\right|^{2} \mathrm{~d} s\right] \geq \frac{1}{2} \mathbb{E}_{\mathrm{P}}\left[\int_{t}^{T}\left|\Psi_{S}\left(X_{S}\right)\right|^{2} \mathrm{~d} t\right]
$$

The desired conclusion follows by plugging (76) in the above equation and some standard calculations. 


\subsection{First derivative of $\mathcal{F}$}

We compute the first derivative of $\mathcal{F}$ along the marginal flow of $\mathrm{Q}$, assuming that $\mathcal{H}(\mathrm{Q} \mid \Gamma(\mathrm{Q}))<+\infty$ and that $\mathrm{Q}$ is Markov. To do this, we use an approach based on optimal transport, and some results of [25]. To be self-contained, we recall the basic notions of optimal transport we need to state the results. We refer to [1] for more details.

Tangent space Let $\mu \in \mathcal{P}_{2}\left(\mathbb{R}^{d}\right)$. The tangent space $\operatorname{Tan}_{\mu} \mathcal{P}_{2}$ at $\mu$ is the closure in $L_{\mu}^{2}$ of

$$
\left\{\nabla \psi ; \psi \in \mathcal{C}_{c}^{\infty}\left(\mathbb{R}^{d}\right)\right\}
$$

Since $L_{\mu}^{2}$ is an Hilbert space, given an arbitrary $\Psi \in L_{\mu}^{2}$, there exists a unique projection $\Pi_{\mu}(\Psi)$ of $\Psi$ onto $\operatorname{Tan}_{\mu} \mathcal{P}_{2}\left(\mathbb{R}^{d}\right)$.

Absolutely continuous curves and velocity field Following [1, Th 8.3.1], we say that a curve $\left(\mu_{t}\right)_{t \in[0, T]} \subseteq \mathcal{P}_{2}\left(\mathbb{R}^{d}\right)$ is absolutely continuous if there exists a Borel measurable vector field $(t, z) \mapsto w_{t}(z)$ such that

- $\left(w_{t}\right)_{t \in[0, T]}$ solves (in the sense of distributions) the continuity equation

$$
\partial_{t} \mu_{t}+\nabla \cdot\left(w_{t} \mu_{t}\right)=0 .
$$

- $w_{t}$ satisfies the integrability condition

$$
\int_{0}^{T}\left(\int_{\mathbb{R}^{d}}\left|w_{t}(z)\right|^{2} \mu_{t}(\mathrm{~d} z)\right)^{1 / 2} \mathrm{~d} t<+\infty .
$$

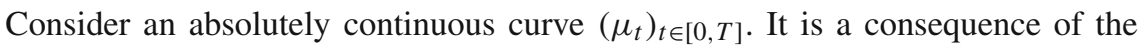
results in Chapter 8, and in particular of Proposition 8.4.5 of [1], that there exist a unique Borel measurable vector field $v_{t}(z)$ solving (77) and such that $z \mapsto v_{t}(z)$ belongs to the tangent space $\operatorname{Tan}_{\mu_{t}} \mathcal{P}_{2}$ for almost every $t \in[0, T]$. We call such $v_{t}$ the (tangent) velocity field of $\left(\mu_{t}\right)_{t \in[0, T]}$.

Remark 4.1 Let $\left(\mu_{t}\right)_{t \in[0, T]}$ be an absolutely continuous curve and $w_{t}(z)$ be in $\mathrm{H}_{-1}\left(\left(\mu_{t}\right)_{t \in[0, T]}\right)$. It is rather easy to see that $z \mapsto w_{t}(z)$ belongs to $\operatorname{Tan}_{\mu_{t}} \mathcal{P}_{2}$ for almost every $t \in[0, T]$.

Throughout the rest of the paper, if $\mathrm{Q} \in \mathcal{P}(\Omega)$ is such that $\mathcal{H}(\mathrm{Q} \mid \Gamma(\mathrm{Q}))<+\infty$, we say that $\mathrm{Q}$ is Markov if $\alpha_{t}^{\mathrm{Q}}$ is $\sigma\left(X_{t}\right)$-measurable for all $t \in[0, T],\left(\alpha_{t}^{\mathrm{Q}}\right)_{t \in[0, T]}$ being defined by (8). In that case we write $\Xi_{t}^{\mathrm{Q}}\left(X_{t}\right)$ instead of $\alpha_{t}^{\mathrm{Q}}$.

Lemma 4.4 Let $\mathrm{Q}$ be such that $\mathcal{H}(\mathrm{Q} \mid \Gamma(\mathrm{Q}))<+\infty$ and Markov. Then 
(i) $\left(\mathrm{Q}_{t}\right)_{t \in[0, T]}$ is an absolutely continuous curve. Its tangent velocity field is given by

$$
v_{t}(z)=-\nabla W * \mathrm{Q}_{t}(z)+\Pi_{\mathrm{Q}_{t}}\left(\Xi_{t}^{\mathrm{Q}}(z)\right)-\frac{1}{2} \nabla \log \mathrm{Q}_{t}(z) .
$$

Moreover,

$$
\int_{0}^{T} \int_{\mathbb{R}^{d}}\left|v_{t}\right|^{2} \mathrm{dQ} \mathrm{Q}_{t} \mathrm{~d} t<+\infty
$$

(ii) The function $t \mapsto \mathcal{F}\left(\mathrm{P}_{t}\right)$ is absolutely continuous and

$$
\begin{aligned}
\forall 0 \leq & s \leq t, \quad \mathcal{F}\left(\mathrm{Q}_{t}\right)-\mathcal{F}\left(\mathrm{Q}_{s}\right)=\int_{s}^{t} \int_{\mathbb{R}^{d}}\left(\nabla \log \mathrm{Q}_{r}\right. \\
& \left.+2 \nabla W * \mathrm{Q}_{r}\right)(z) \cdot v_{r}(z) \mathrm{Q}_{r}(\mathrm{~d} z) \mathrm{d} r .
\end{aligned}
$$

Proof Proof of (i) To show that $\left(\mathrm{Q}_{t}\right)_{t \in[0, T]}$ is absolutely continuous it suffices to show that there exists a distributional solution of the continuity equation

$$
\partial_{t} \mathrm{Q}_{t}+\nabla \cdot\left(w_{t} \mathrm{Q}_{t}\right)=0
$$

with the property that

$$
\int_{0}^{T}\left(\int_{\mathbb{R}^{d}}\left|w_{t}(z)\right|^{2} \mathrm{Q}_{t}(z)\right)^{1 / 2} \mathrm{~d} t<+\infty
$$

Let now $\varphi \in \mathcal{C}_{c}^{\infty}(] 0, T\left[\times \mathbb{R}^{d}\right)$. Using Itô's formula and taking expectation we obtain

$$
\int_{0}^{T} \int_{\mathbb{R}^{d}}\left(\nabla \varphi(t, z)\left(-\nabla W * \mathrm{Q}_{t}(z)+\Xi_{t}^{\mathrm{Q}}(z)\right)+\frac{1}{2} \Delta \varphi(t, z)+\partial_{t} \varphi(t, z)\right) \mathrm{Q}_{t}(\mathrm{~d} z)=0 .
$$

Lemma 5.2 in the appendix grants that under the current assumptions $\mathcal{H}\left(\mathrm{Q} \mid \mathrm{R}^{\mu^{\text {in }}}\right)<$ $+\infty$, where $\mathrm{R}^{\mu^{\text {in }}}$ is the Wiener measure started at $\mu^{\text {in }}$. But then, using [25, Thm 3.10] ${ }^{10}$ we obtain that $\log \mathrm{Q}_{t}$ is an absolutely continuous function for almost every $t$ and that $(t, z) \mapsto \nabla \log \mathrm{Q}_{t}(z)$ belongs to $\mathrm{H}_{-1}\left(\left(\mathrm{Q}_{t}\right)_{t \in[0, T]}\right)$. Therefore we can use integration by parts in (83) to obtain

$$
\forall t \in[0, T], \quad \frac{1}{2} \int_{\mathbb{R}^{d}} \nabla \varphi(t, z) \mathrm{Q}_{t}(\mathrm{~d} z)=-\frac{1}{2} \int_{\mathbb{R}^{d}} \Delta \log \varphi(t, z) \cdot \nabla \log \mathrm{Q}_{t}(z) \mathrm{Q}_{t}(\mathrm{~d} z)
$$

which gives, using the definition of the projection operator $\Pi_{\mathrm{Q}_{t}}$, that the rhs of (78) solves the continuity equation in the sense of distributions. Next, we observe that (8)

\footnotetext{
$\overline{10}$ Strictly speaking, Föllmer's result is only concerned with the case $\mu^{\text {in }}=\delta_{0}$. However, a simple adaptation of his argument show that its validity extends to any $\mu^{\text {in }}$ satisfying (H2). 
grants that $\Pi_{\mathrm{Q}_{t}}\left(\Xi^{\mathrm{Q}}(t, z)\right) \in \mathrm{H}_{-1}\left((\mathrm{Q})_{t \in[0, T]}\right)$. We have already shown that $\nabla \log \mathrm{Q}_{t} \in$ $\mathrm{H}_{-1}\left((\mathrm{Q})_{t \in[0, T]}\right)$, and (54) used with $\bar{b}=-\nabla W * \mathrm{Q}_{t}(z)$ yields that $-\nabla W * \mathrm{Q}_{t}(z) \in$ $\mathrm{H}_{-1}\left((\mathrm{Q})_{t \in[0, T]}\right)$. Thus $v_{t}(z) \in \mathrm{H}_{-1}\left((\mathrm{Q})_{t \in[0, T]}\right)$ as well, which gives (82) and (79). Finally, Remark 4.1 yields that $\left(v_{t}\right)_{t \in[0, T]}$ is indeed the tangent velocity field.

Proof of (ii) From point (i) we know that $z \mapsto \nabla \log \mathrm{Q}_{t}(z)$ belongs to $L_{\mathrm{Q}_{t}}^{2}$ for almost every $t$; this implies that $\nabla \log \mathrm{Q}_{t}+2 \nabla W * \mathrm{Q}_{t}$ belongs to the subdifferential of $\mathcal{F}$ at $\mathrm{Q}_{t}$ for almost every $t$ (see e.g. [1, Thm. 10.4.13]). The chain rule [1, sec. E, pp. 233-234] gives the desired result (80), provided its hypothesis are verified. We have to check that (a) $\left(\mathrm{Q}_{t}\right)_{t \in[0, T]}$ is an absolutely continuous curve and $\mathcal{F}\left(\mathrm{Q}_{t}\right)<+\infty$ for all $t \in[0, T]$, (b) $\mathcal{F}(\cdot)$ is displacement $\lambda$-convex for some $\lambda \in \mathbb{R}$, and (c) that

$$
\int_{0}^{T}\left(\int_{\mathbb{R}^{d}}\left|v_{t}\right|^{2} \mathrm{dQ}_{t}\right)^{1 / 2}\left(\int_{\mathbb{R}^{d}}\left|\nabla \log \mathrm{Q}_{t}+2 \nabla W * \mathrm{Q}_{t}\right|^{2} \mathrm{dQ} t\right)^{1 / 2} \mathrm{~d} t<+\infty
$$

To wit, (a) follows from point (i) and the fact that $\mathcal{H}(\mathrm{Q} \mid \Gamma(\mathrm{Q}))<+\infty$, and (b) is a consequence of displacement convexity of the entropy and (H1). Finally, (c) is granted by (79) and the fact that $\nabla \log \mathrm{Q}_{t}(z)+2 \nabla W * \mathrm{Q}_{t}(z)$ belongs to $\mathrm{H}_{-1}\left(\left(\mathrm{Q}_{t}\right)_{t \in[0, T]}\right)$ (see the proof of (i)).

\subsection{Time reversal}

For $\mathrm{Q} \in \mathcal{P}(\Omega)$ the time reversal $\hat{\mathrm{Q}}$ is the law of the time reversed process $\left(X_{T-t}\right)_{t \in[0, T]}$. In this section we derive an expression for $\mathcal{H}(\hat{\mathrm{Q}} \mid \Gamma(\hat{\mathrm{Q}}))$ and use it to derive the bound (91) below, which plays a fundamental role in the proof of Theorem 4.1.

Proposition 4.1 Let $\mathrm{Q} \in \mathcal{P}_{1}(\Omega)$ be Markov and such that $\mathcal{H}(\mathrm{Q} \mid \Gamma(\mathrm{Q}))<+\infty$.

(i) If $\mathrm{Q}_{0}=\mu^{\text {in }}, \mathrm{Q}_{T}=\mu^{\text {fin }}$ then $\mathcal{H}(\hat{\mathrm{Q}} \mid \Gamma(\hat{\mathrm{Q}}))<+\infty$ as well and

$$
\mathcal{H}(\hat{\mathrm{Q}} \mid \Gamma(\hat{\mathrm{Q}}))=\mathcal{H}(\mathrm{Q} \mid \Gamma(\mathrm{Q}))+\mathcal{F}\left(\mu^{\mathrm{in}}\right)-\mathcal{F}\left(\mu^{\mathrm{fin}}\right)
$$

(ii) If $\mathrm{Q}_{0}=\mu^{\mathrm{fin}}, \mathrm{Q}_{T}=\mu^{\text {in }}$ then $\mathcal{H}(\hat{\mathrm{Q}} \mid \Gamma(\hat{\mathrm{Q}}))<+\infty$ as well and

$$
\mathcal{H}(\mathrm{Q} \mid \Gamma(\mathrm{Q}))=\mathcal{H}(\hat{\mathrm{Q}} \mid \Gamma(\hat{\mathrm{Q}}))+\mathcal{F}\left(\mu^{\mathrm{in}}\right)-\mathcal{F}\left(\mu^{\mathrm{fin}}\right)
$$

Proof We only prove (i), (ii) being completely analogous. Recalling (see Lemma 5.2) that $\mathcal{H}(\mathrm{Q} \mid \Gamma(\mathrm{Q}))<+\infty$ implies $\mathcal{H}\left(\mathrm{Q} \mid \mathrm{R}^{\mu^{\text {in }}}\right)<+\infty$, we can use [25, Thm. 3.10, Eq. 3.9] to obtain that there exist a Borel measurable vector field $\hat{b}_{t}(x)$ such that

$$
X_{t}-\int_{0}^{t} \hat{b}_{s}\left(X_{s}\right) \mathrm{d} s
$$

is a Brownian motion under $\hat{\mathrm{P}}$ and that

$$
\hat{\mathrm{Q}}-\text { a.s. } \quad \hat{b}_{t}\left(X_{t}\right)=-b_{T-t}\left(X_{t}\right)+\nabla \log \mathrm{Q}_{T-t}\left(X_{t}\right) \quad \forall t \in[0, T],
$$


where $b_{t}(z)$ is the drift of $\mathrm{Q}$, that, in view of Lemma 1.1 we write as $-\nabla W * \mathrm{Q}_{t}+\Xi_{t}^{\mathrm{Q}}(z)$. Thus, we deduce that under $\hat{Q}$ we have that

$$
X_{t}-\int_{0}^{t}-\nabla W * \hat{\mathrm{Q}}_{s}\left(X_{s}\right)+\hat{\Xi}_{s}^{\mathrm{Q}}\left(X_{s}\right) \mathrm{d} s
$$

is a Brownian motion, where

$$
\begin{aligned}
\hat{\mathrm{Q}}-\text { a.s. } \quad \hat{\Xi}_{t}^{\mathrm{Q}}\left(X_{t}\right)= & -\Xi_{T-t}^{\mathrm{Q}}\left(X_{t}\right) \\
& +\nabla \log \mathrm{Q}_{T-t}\left(X_{t}\right)+2 \nabla W * \mathrm{Q}_{T-t}\left(X_{t}\right) \quad \forall t \in[0, T] .
\end{aligned}
$$

In the proof of Lemma 4.4, it was shown that $\left(\nabla \log \mathrm{Q}_{t}\right)_{t \in[0, T]},\left(\nabla W * \mathrm{Q}_{\text {. }}\right)_{t \in[0, T]}$ and $\left(\Xi_{t}^{\mathrm{Q}}\right)_{t \in[0, T]}$ are all in $\mathrm{H}_{-1}\left(\left(\mathrm{Q}_{t}\right)_{t \in[0, T]}\right)$. This implies that $\left(\hat{\Xi}_{t}^{\mathrm{Q}}\right)_{t \in[0, T]} \in$ $\mathrm{H}_{-1}\left(\left(\hat{\mathrm{Q}}_{t}\right)_{t \in[0, T]}\right)$ as well. But then using $(i i) \Rightarrow(i)$ in Lemma 3.6 for the choice $\bar{b}(t, z)=-\nabla W * \hat{\mathrm{Q}}_{t}(z)$ we get that $\mathcal{H}(\hat{\mathrm{Q}} \mid \Gamma(\hat{\mathrm{Q}}))<+\infty$ and

$$
\mathcal{H}(\hat{\mathrm{Q}} \mid \Gamma(\hat{\mathrm{Q}}))=\frac{1}{2} \mathbb{E}_{\hat{\mathrm{Q}}}\left[\int_{0}^{T}\left|\hat{\Xi}_{t}^{\mathrm{Q}}\left(X_{t}\right)\right|^{2} \mathrm{~d} t\right] .
$$

Using (87) in the above equation we get

$$
\begin{aligned}
\mathcal{H}(\hat{\mathrm{Q}} \mid \Gamma(\hat{\mathrm{Q}})) & \\
= & \frac{1}{2} \mathbb{E}_{\hat{\mathrm{Q}}}\left[\int_{0}^{T}\left|\Xi_{T-t}^{\mathrm{Q}}\left(X_{t}\right)-\left(\nabla \log \mathrm{Q}_{T-t}\left(X_{t}\right)+2 \nabla W * \mathrm{Q}_{T-t}\left(X_{t}\right)\right)\right|^{2} \mathrm{~d} t\right] \\
= & \frac{1}{2} \mathbb{E}_{\mathrm{Q}}\left[\int_{0}^{T}\left|\Xi_{t}^{\mathrm{Q}}\left(X_{t}\right)\right|^{2} \mathrm{~d} t\right] \\
& +\frac{1}{2} \mathbb{E}_{\mathrm{Q}}\left[\int_{0}^{T}\left(\nabla \log \mathrm{Q}_{t}\left(X_{t}\right)+2 \nabla W * \mathrm{Q}_{t}\left(X_{t}\right)\right) .\right. \\
& \left.\left(-2 \Xi_{t}^{\mathrm{Q}}\left(X_{t}\right)+\nabla \log \mathrm{Q}_{t}\left(X_{t}\right)+2 \nabla W * \mathrm{Q}_{t}\left(X_{t}\right)\right) \mathrm{d} t\right] \\
& \stackrel{(78)}{=} \mathcal{H}(\mathrm{Q} \mid \Gamma(\mathrm{Q}))-\mathbb{E}_{\mathrm{Q}}\left[\int_{0}^{T}\left(\nabla \log \mathrm{Q}_{t}\left(X_{t}\right)+2 \nabla W * \mathrm{Q}_{t}\left(X_{t}\right)\right) \cdot v_{t}\left(X_{t}\right) \mathrm{d} t\right] .
\end{aligned}
$$

The conclusion follows from point (ii) of Lemma 4.4.

A consequence of Proposition 4.1 is that optimality is preserved under time reversal. Lemma 4.5 Let $\mathrm{P}$ be an optimizer for (MFSP). Then $\hat{\mathrm{P}}$ optimizes

$$
\inf \left\{\mathcal{H}(\mathrm{Q} \mid \Gamma(\mathrm{Q})): \mathrm{Q} \in \mathcal{P}_{1}(\Omega), \mathrm{Q}_{0}=\mu^{\mathrm{fin}}, \mathrm{Q}_{T}=\mu^{\mathrm{in}}\right\}
$$

Proof Let us observe that since (H2) makes no distinction between $\mu^{\text {in }}$ and $\mu^{\text {fin }}$, the problem (88) admits at least an optimal solution by Proposition 1.1. Applying Proposition 3.1 inverting the roles of $\mu^{\text {in }}, \mu^{\text {fin }}$ we get that the optimizers of (88) are 
Markov. So it suffices to show that for any Markov Q admissible for (88) we have $\mathcal{H}(\mathrm{Q} \mid \Gamma(\mathrm{Q})) \geq \mathcal{H}(\hat{\mathrm{P}} \mid \Gamma(\hat{\mathrm{P}}))$. Take any such $\mathrm{Q}$. We have

$$
\begin{gathered}
\mathcal{H}(\mathrm{Q} \mid \Gamma(\mathrm{Q})) \stackrel{\text { Prop. }}{\stackrel{4.1(i i)}{=}} \mathcal{H}(\hat{\mathrm{Q}} \mid \Gamma(\hat{\mathrm{Q}}))+\mathcal{F}\left(\mu^{\text {in }}\right)-\mathcal{F}\left(\mu^{\mathrm{fin}}\right) \\
\stackrel{\text { Opt. of } \mathrm{P}}{\geq} \mathcal{H}(\mathrm{P} \mid \Gamma(\mathrm{P}))+\mathcal{F}\left(\mu^{\mathrm{in}}\right)-\mathcal{F}\left(\mu^{\mathrm{fin}}\right) \\
\stackrel{\text { Prop. }}{=}{ }^{=}{ }^{(i)} \mathcal{H}(\hat{\mathrm{P}} \mid \Gamma(\hat{\mathrm{P}})) .
\end{gathered}
$$

\subsection{Functional inequalities: proofs and the behaviour of $\mathcal{F}$}

The goal of this section is to prove Theorem 1.6 as well as the Talagrand and HWI inequalities. The latter are colloraries of the following new result concerning the behaviour of $\mathcal{F}$ along bridges:

Theorem 4.1 Assume (H1)-(H4) and let $T>0$ be fixed. If $\mathrm{P}$ is an optimizer for (MFSP), then for all $t \in[0, T]$ we have

$$
\begin{aligned}
\mathcal{F}\left(\mathrm{P}_{t}\right) \leq & \frac{\exp (2 \kappa(T-t))-1}{\exp (2 \kappa T)-1} \mathcal{F}\left(\mu^{\mathrm{in}}\right)+\frac{\exp (2 \kappa T)-\exp (2 \kappa(T-t))}{\exp (2 \kappa T)-1} \mathcal{F}\left(\mu^{\mathrm{fin}}\right) \\
& -\frac{(\exp (2 \kappa(T-t))-1)(\exp (2 \kappa t)-1)}{\exp (2 \kappa T)-1} \mathscr{C}_{T}\left(\mu^{\mathrm{in}}, \mu^{\mathrm{fin}}\right) .
\end{aligned}
$$

This bound generalizes to the mean field setup the results of [17], and may be seen as a rigorous version of some of the heuristic arguments put forward in [28] and [34], upon slightly modifying the definition of $\mathscr{C}_{T}$.

\subsubsection{Proof of Theorem 4.1}

Using a time reversal argument, we prove the bound (91) which is the key ingredient of the proof of Theorem 4.1 together with the bound for the correction term (70).

The backward corrector $\hat{\Psi}$ is obtained by the same argument used in Proposition 4.1, replacing (MFSP) with (88) to obtain that there exist a Borel measurable vector field $\hat{\Psi}_{t}(z) \in \mathrm{H}_{-1}\left(\left(\hat{\mathrm{P}}_{t}\right)_{t \in[0, T]}\right)$ such that

$$
X_{t}-\int_{0}^{t}\left(-\nabla W * \hat{\mathrm{P}}_{s}\left(X_{s}\right)+\hat{\Psi}_{s}\left(X_{s}\right)\right) \mathrm{d} s
$$

is a Brownian motion under $\hat{\mathrm{P}}$. Moreover, the following relation holds

$$
\begin{aligned}
& \hat{\mathrm{P}}-\text { a.s. } \hat{\Psi}_{t}\left(X_{t}\right)=-\Psi_{T-t}\left(X_{t}\right)+\nabla \log \mathrm{P}_{T-t}\left(X_{t}\right) \\
& \quad+2 \nabla W * \mathrm{P}_{T-t}\left(X_{t}\right) \quad \forall t \in[0, T] .
\end{aligned}
$$


Lemma 4.6 Assume (H3), (H4) and let $\mathrm{P}$ be an optimizer for (MFSP). Then

$$
\begin{gathered}
\mathcal{F}\left(\mathrm{P}_{r}\right)+\frac{1}{2} \mathbb{E}_{\mathrm{P}}\left[\int_{r}^{T}\left|\Psi_{s}\left(X_{s}\right)\right|^{2} \mathrm{~d} s\right] \leq \frac{\exp (2 \kappa(T-r))-1}{\exp (2 \kappa T)-1} \mathscr{C}_{T}\left(\mu^{\mathrm{in}}, \mu^{\mathrm{fin}}\right) \\
+\frac{\exp (2 \kappa(T-r))-1}{\exp (2 \kappa T)-1} \mathcal{F}\left(\mu^{\mathrm{in}}\right)+\frac{\exp (2 \kappa T)-\exp (2 \kappa(T-r))}{\exp (2 \kappa T)-1} \mathcal{F}\left(\mu^{\mathrm{fin}}\right)
\end{gathered}
$$

and

$$
\frac{1}{2} \mathbb{E}_{\mathrm{P}}\left[\left|\hat{\Psi}_{T-r}\left(X_{r}\right)\right|^{2}\right] \leq \frac{2 \kappa \mathscr{C}_{T}\left(\mu^{\mathrm{fin}}, \mu^{\text {in }}\right)}{\exp (2 \kappa r)-1}
$$

hold for all $r \in(0, T)$.

Proof Using (78) we can rewrite the above equation (90) as

$$
\hat{\mathrm{P}}-\text { a.s. } \quad \hat{\Psi}_{t}\left(X_{t}\right)=\Psi_{T-t}\left(X_{t}\right)-2 v_{T-t}\left(X_{t}\right) \quad \forall t \in[0, T]
$$

From Proposition 4.5 we also know that $\hat{\mathrm{P}}$ is optimal for (88) and hence that $\mathcal{H}(\hat{\mathrm{P}} \mid \Gamma(\hat{\mathrm{P}}))=\mathscr{C}_{T}\left(\mu^{\mathrm{fin}}, \mu^{\mathrm{in}}\right)$. Therefore, by inverting again the roles of $\mu^{\text {in }}$ and $\mu^{\mathrm{fin}}$, we can use Lemma 4.3 for the problem (88) setting $t=T-r$ to derive that

$$
\frac{1}{2} \mathbb{E}_{\hat{\mathrm{P}}}\left[\int_{0}^{T-r}\left|\hat{\Psi}_{s}\left(X_{S}\right)\right|^{2} \mathrm{~d} s\right] \leq \frac{\exp (2 \kappa(T-r))-1}{\exp (2 \kappa T)-1} \mathcal{H}(\hat{\mathrm{P}} \mid \Gamma(\hat{\mathrm{P}})) .
$$

Thanks to (93) we can write

$$
\begin{aligned}
& \frac{1}{2} \mathbb{E}_{\hat{\mathrm{P}}}\left[\int_{0}^{T-r}\left|\hat{\Psi}_{s}\left(X_{S}\right)\right|^{2} \mathrm{~d} s\right]=\frac{1}{2} \mathbb{E}_{\mathrm{P}}\left[\int_{r}^{T}\left|\Psi_{s}\left(X_{S}\right)\right|^{2} \mathrm{~d} s\right] \\
& -\mathbb{E}_{\mathrm{P}}\left[\int_{r}^{T}\left(2 \Psi_{s}\left(X_{S}\right)-2 v_{s}\left(X_{S}\right)\right) v_{s}\left(X_{S}\right) \mathrm{d} s\right] \\
& \stackrel{(78)+\stackrel{\Psi}{=} \in \mathrm{H}_{-1}}{=} \frac{1}{2} \mathbb{E}_{\mathrm{P}}\left[\int_{r}^{T}\left|\Psi_{S}\left(X_{S}\right)\right|^{2} \mathrm{~d} s\right] \\
& -\mathbb{E}_{\mathrm{P}}\left[\int_{r}^{T}\left(\nabla \log \mathrm{P}_{S}\left(X_{S}\right)+2 \nabla W * \mathrm{P}_{S}\left(X_{S}\right)\right) \cdot v_{S}\left(X_{S}\right) \mathrm{d} s\right] \\
& \stackrel{(80)}{=} \frac{1}{2} \mathbb{E}_{\mathrm{P}}\left[\int_{r}^{T}\left|\Psi_{s}\left(X_{s}\right)\right|^{2} \mathrm{~d} s\right]+\mathcal{F}\left(\mathrm{P}_{r}\right)-\mathcal{F}\left(\mu^{\mathrm{fin}}\right) \text {. }
\end{aligned}
$$

The bound (91) follows by plugging in (95) into (94) using the above equation, (84) and recalling that $\mathcal{H}(\mathrm{P} \mid \Gamma(\mathrm{P}))=\mathscr{C}_{T}\left(\mu^{\mathrm{in}}, \mu^{\text {fin }}\right)$. The proof of (92) goes along the same lines: Since $\hat{\mathrm{P}}$ is optimal for (88) we also get from Lemma 4.3, and in particular from (71) for the choice $t=T-r$ that 


$$
\frac{1}{2} \mathbb{E}_{\hat{\mathrm{P}}}\left[\left|\hat{\Psi}_{T-r}\left(X_{T-r}\right)\right|^{2}\right] \leq \frac{2 \kappa \mathscr{C}_{T}\left(\mu^{\mathrm{fin}}, \mu^{\mathrm{in}}\right)}{\exp (2 \kappa r)-1}
$$

Now the proof of Theorem 4.1 and its corollaries in the introduction is an easy task, given all the preparatory work.

Proof of Theorem 4.1 It amounts to add (70) and (91) with the choice $r=t$, and use the relation

$$
\mathcal{H}(\mathrm{P} \mid \Gamma(\mathrm{P}))=\frac{1}{2} \mathbb{E}_{\mathrm{P}}\left[\int_{0}^{T}\left|\Psi_{t}\left(X_{t}\right)\right|^{2} \mathrm{~d} t\right]=\mathscr{C}_{T}\left(\mu^{\mathrm{in}}, \mu^{\mathrm{fin}}\right) .
$$

Proof of Corollary 1.3 It follows from Theorem 4.1 (Eq. (89)), observing that $\mathcal{F}\left(\mathrm{P}_{t}\right) \geq$ 0 .

Proof of Corollary 1.4 Combining (90), (93), (31) we get that

$$
\int_{\mathbb{R}^{d}}\left|v_{t}\right|^{2}(x) \mathrm{P}_{t}(\mathrm{~d} x)=-\mathscr{E}_{\mathrm{P}}\left(\mu^{\mathrm{in}}, \mu_{\infty}\right)+\frac{1}{4} \mathcal{I}_{\mathcal{F}}\left(\mathrm{P}_{t}\right) .
$$

Using the above relation, Cauchy Schwartz inequality and the continuity of $\mathcal{I}_{\mathcal{F}}\left(\mathrm{P}_{t}\right)$ in a neighborhood of $0,(80)$ we get that

$$
\liminf _{t \rightarrow 0} \frac{1}{t}\left(\mathcal{F}\left(\mathrm{P}_{t}\right)-\mathcal{F}\left(\mathrm{P}_{0}\right)\right) \geq-\left(\mathcal{I}_{\mathcal{F}}\left(\mu^{\mathrm{in}}\right)\left(\frac{1}{4} \mathcal{I}_{\mathcal{F}}\left(\mu^{\mathrm{in}}\right)-\mathscr{E}_{\mathrm{P}}\left(\mu^{\mathrm{in}}, \mu_{\infty}\right)\right)\right)^{1 / 2} .
$$

Consider now the bound (89). Observing that $\mathcal{F}\left(\mu_{\infty}\right)=0$, subtracting $\mathcal{F}\left(\mu^{\text {in }}\right)$ on both sides, dividing by $t$, letting $t \rightarrow 0$, using (96) and finally rearranging the resulting terms we get (28).

\subsubsection{Proof of Theorem 1.6}

We prove here Theorem 1.6 of the introduction. In the proof we will write

$$
\int_{\mathbb{R}^{d}} \nabla^{2} W\left(\hat{X}_{t}-y\right) \cdot\left(\hat{\Psi}_{t}\left(\hat{X}_{t}\right)-\hat{\Psi}_{t}(y)\right) \hat{\mathrm{P}}_{t}(\mathrm{~d} y),
$$

instead of

$$
\tilde{\mathbb{E}}_{\tilde{\mathrm{P}}}\left[\nabla^{2} W\left(X_{S}-\tilde{X}_{S}\right) \cdot\left(\Psi_{s}\left(X_{S}\right)-\Psi_{s}\left(\tilde{X}_{S}\right)\right)\right],
$$

which is used in the rest of the article. This is done in order to better deal with time reversal. 
Proof of Theorem 1.6 Let $M_{t}$ be the martingale defined at (14). Since $\hat{\mathrm{P}}$ is optimal for (88), from Proposition 3.2 we get that

$$
\hat{M}_{t}=\hat{\Psi}_{t}\left(X_{t}\right)-\int_{0}^{t} \int_{\mathbb{R}^{d}} \nabla^{2} W\left(\hat{X}_{s}-y\right) \cdot\left(\hat{\Psi}_{s}\left(X_{s}\right)-\hat{\Psi}_{s}(y)\right) \hat{\mathrm{P}}_{s}(\mathrm{~d} y) \mathrm{d} s
$$

is an $L^{2}$-martingale on $[0, T[$ under $\hat{\mathrm{P}}$. We define the stochastic processes

$$
A_{t}:=\int_{\mathbb{R}^{d}} \nabla^{2} W\left(X_{s}-y\right) \cdot\left(\Psi_{s}\left(X_{s}\right)-\Psi_{s}(y)\right) \mathrm{P}_{s}(\mathrm{~d} y)
$$

and

$$
\hat{A}_{t}:=\int_{\mathbb{R}^{d}} \nabla^{2} W\left(\hat{X}_{s}-y\right) \cdot\left(\hat{\Psi}_{s}\left(\hat{X}_{s}\right)-\hat{\Psi}_{s}(y)\right) \hat{\mathrm{P}}_{s}(\mathrm{~d} y)
$$

We have, using the Markovianity of both $\mathrm{P}$ and $\hat{\mathrm{P}}$, that

$$
\begin{aligned}
\mathbb{E}_{\mathrm{P}}\left[\Psi_{t}\left(X_{t}\right) \cdot \hat{\Psi}_{T-t}\left(\hat{X}_{T-t}\right)\right] \\
=\mathbb{E}_{\mathrm{P}}\left[\left(M_{t}+\int_{0}^{t} A_{s} \mathrm{~d} s\right) \cdot\left(\hat{M}_{T-t}+\int_{0}^{T-t} \hat{A}_{s} \mathrm{~d} s\right)\right] \\
=\mathbb{E}_{\mathrm{P}}\left[\left(\mathbb{E}_{\mathrm{P}}\left[M_{T} \mid X_{[0, t]}\right]+\int_{0}^{t} A_{s} \mathrm{~d} s\right) \cdot\left(\mathbb{E}_{\mathrm{P}}\left[\hat{M}_{T} \mid \hat{X}_{[0, T-t]}\right]+\int_{0}^{T-t} \hat{A}_{s} \mathrm{~d} s\right)\right] \\
=\mathbb{E}_{\mathrm{P}}\left[\mathbb{E}_{\mathrm{P}}\left[\Psi_{T}\left(X_{T}\right)-\int_{t}^{T} A_{s} \mathrm{~d} s \mid X_{[0, t]}\right] \cdot \mathbb{E}_{\mathrm{P}}\left[\hat{\Psi}_{T}\left(\hat{X}_{T}\right)-\int_{T-t}^{T} \hat{A}_{s} \mathrm{~d} \mid \hat{X}_{[0, T-t]}\right]\right] \\
=\mathbb{E}_{\mathrm{P}}\left[\mathbb{E}_{\mathrm{P}}\left[\Psi_{T}\left(X_{T}\right)-\int_{t}^{T} A_{s} \mathrm{~d} s \mid X_{t}\right] \cdot \mathbb{E}_{\mathrm{P}}\left[\hat{\Psi}_{T}\left(\hat{X}_{T}\right)-\int_{T-t}^{T} \hat{A}_{s} \mathrm{~d} s \mid \hat{X}_{t}\right]\right] \\
=\mathbb{E}_{\mathrm{P}}\left[\left(\Psi_{T}\left(X_{T}\right)-\int_{t}^{T} A_{s} \mathrm{~d} s\right) \cdot\left(\hat{\Psi}_{T}\left(\hat{X}_{T}\right)-\int_{T-t}^{T} \hat{A}_{s} \mathrm{~d} s\right)\right] .
\end{aligned}
$$

Therefore,

$$
\begin{aligned}
& \frac{\mathrm{d}}{\mathrm{d} t} \mathbb{E}_{\mathrm{P}}\left[\Psi_{t}\left(X_{t}\right) \cdot \hat{\Psi}_{T-t}\left(\hat{X}_{T-t}\right)\right] \\
& \quad=\mathbb{E}_{\mathrm{P}}\left[-A_{t} \cdot\left(\hat{\Psi}_{T}\left(\hat{X}_{T}\right)-\int_{T-t}^{T} \hat{A}_{s}\right)+\hat{A}_{T-t} \cdot\left(\Psi_{T}\left(X_{T}\right)-\int_{t}^{T} A_{s} \mathrm{~d} s\right)\right] \\
& \quad=\mathbb{E}_{\mathrm{P}}\left[-A_{t} \cdot\left(\hat{M}_{T}-\hat{M}_{T-t}+\hat{\Psi}_{T-t}\left(\hat{X}_{T-t}\right)\right)+\hat{A}_{T-t} \cdot\left(M_{T}-M_{t}+\Psi_{t}\left(X_{t}\right)\right)\right] .
\end{aligned}
$$

Taking conditional expectation w.r.t. $\sigma\left(X_{[0, t]}\right)$ and using that both $A_{t}$ and $\hat{A}_{T-t}$ are $X_{[0, t]}$-measurable we get that the above expression equals

$$
\mathbb{E}_{\mathrm{P}}\left[-A_{t} \cdot \hat{\Psi}_{T-t}\left(\hat{X}_{T-t}\right)+\hat{A}_{T-t} \cdot \Psi_{t}\left(X_{t}\right)\right]
$$


Using the fact that $W$ is symmetric and the definition of $A_{t}, \hat{A}_{T-t}$, one easily obtains that the latter expression is worth 0 . Indeed it holds that

$$
\begin{aligned}
\mathbb{E}_{\mathrm{P}}\left[A_{t} \cdot \hat{\Psi}_{T-t}\left(\hat{X}_{T-t}\right)\right]= & \mathbb{E}_{\mathrm{P}}\left[\hat{A}_{T-t} \cdot \Psi_{t}\left(X_{t}\right)\right] \\
= & \int_{\mathbb{R}^{d} \times \mathbb{R}^{d}}\left(\hat{\Psi}_{T-t}(x)-\hat{\Psi}_{T-t}(y)\right) \cdot \nabla^{2} W(x-y) \cdot\left(\Psi_{t}(x)\right. \\
& \left.-\Psi_{t}(y)\right) \mathrm{P}_{t}(\mathrm{~d} x) \mathrm{P}_{t}(\mathrm{~d} y) .
\end{aligned}
$$

The proof that the function (31) is constant on $(0, T)$ is now concluded. In order to establish (32) we set $t=T / 2$ in (31) and Cauchy Schwartz to get that

$$
\left|\mathscr{E}_{\mathrm{P}}\left(\mu^{\mathrm{in}}, \mu^{\mathrm{fin}}\right)\right| \leq\left(\mathbb{E}_{\mathrm{P}}\left[\left|\Psi_{T / 2}\left(X_{T / 2}\right)\right|^{2}\right] \mathbb{E}_{\mathrm{P}}\left[\left|\hat{\Psi}_{T / 2}\left(\hat{X}_{T / 2}\right)\right|^{2}\right]\right)^{1 / 2}
$$

The desired conclusion follows from (71) and (92).

\subsection{Convergence to equilibrium: proofs}

\subsubsection{Proof of Theorem 1.4}

Proof of Theorem 1.4 Lemma 4.4 provides with

$$
\begin{aligned}
\frac{\mathrm{d}}{\mathrm{d} t} \mathcal{F}\left(\mathrm{P}_{t}\right) & \stackrel{(80)}{=} \mathbb{E}_{\mathrm{P}}\left[\left(\nabla \log \mathrm{P}_{t}\left(X_{t}\right)+2 \nabla W * \mathrm{P}_{t}\left(X_{t}\right)\right) \cdot v_{t}\left(X_{t}\right)\right] \\
& \stackrel{(90)+(93)}{=} \frac{1}{2} \mathbb{E}_{\mathrm{P}}\left[\left(\Psi_{t}\left(X_{t}\right)+\hat{\Psi}_{T-t}\left(\hat{X}_{T-t}\right)\right) \cdot\left(\Psi_{t}\left(X_{t}\right)-\hat{\Psi}_{T-t}\left(\hat{X}_{T-t}\right)\right)\right] \\
& =\frac{1}{2} \mathbb{E}_{\mathrm{P}}\left[\left|\Psi_{t}\left(X_{t}\right)\right|^{2}-\left|\hat{\Psi}_{T-t}\left(\hat{X}_{T-t}\right)\right|^{2}\right] .
\end{aligned}
$$

Reasoning as in the proof of Lemma 4.3 we get that both $\mathbb{E}_{\mathrm{P}}\left[\left|\Psi_{t}\left(X_{t}\right)\right|^{2}\right]$ and $\mathbb{E}_{\mathrm{P}}\left[\left|\hat{\Psi}_{T-t}\left(\hat{X}_{T-t}\right)\right|^{2}\right]$ are differentiable as functions of $t$ in the open interval $(0, T)$. Moreover

$$
\begin{aligned}
\frac{1}{2} & \frac{\mathrm{d}}{\mathrm{d} t} \mathbb{E}_{\mathrm{P}}\left[\left|\Psi_{t}\left(X_{t}\right)\right|^{2}-\left|\hat{\Psi}_{T-t}\left(\hat{X}_{T-t}\right)\right|^{2}\right] \\
& \stackrel{(72)+(75)}{\geq} \kappa \mathbb{E}_{\mathrm{P}}\left[\left|\Psi_{t}\left(X_{t}\right)\right|^{2}+\left|\hat{\Psi}_{T-t}\left(\hat{X}_{T-t}\right)\right|^{2}\right] \\
\quad & \kappa \mathbb{E}_{\mathrm{P}}\left[\left|\Psi_{t}\left(X_{t}\right)+\hat{\Psi}_{T-t}\left(\hat{X}_{T-t}\right)\right|^{2}-2 \Psi_{t}\left(X_{t}\right) \cdot \hat{\Psi}_{T-t}\left(\hat{X}_{T-t}\right)\right] \\
& \stackrel{(87)}{=} \kappa \mathbb{E}_{\mathrm{P}}\left[\left|\nabla \log \mathrm{P}_{t}\left(X_{t}\right)+2 \nabla W * \mathrm{P}_{t}\left(X_{t}\right)\right|^{2}\right]-2 \kappa \mathscr{E}_{\mathrm{P}}\left(\mu^{i n}, \mu^{f i n}\right) .
\end{aligned}
$$

The $\kappa$-convexity of $W$ and the fact that the center of mass $\mathbb{E}_{\mathrm{P}}\left[X_{t}\right]$ is constant (see Lemma 4.2) allow to use the logarithmic Sobolev inequality [12, (ii), Thm 2.2] to 
obtain $^{11}$

$$
\kappa \mathbb{E}_{\mathrm{P}}\left[\left|\nabla \log \mathrm{P}_{t}\left(X_{t}\right)+2 \nabla W * \mathrm{P}_{t}\left(X_{t}\right)\right|^{2}\right] \geq 4 \kappa^{2} \mathcal{F}\left(\mathrm{P}_{t}\right)
$$

Thus we have proven that for almost every $t \in[0, T]$

$$
\frac{\mathrm{d}}{\mathrm{d} t^{2}} \mathcal{F}\left(\mathrm{P}_{t}\right) \geq 4 \kappa^{2} \mathcal{F}\left(\mathrm{P}_{t}\right)-2 \kappa \mathscr{E}_{\mathrm{P}}\left(\mu^{i n}, \mu^{f i n}\right)
$$

from which (22) follows by integrating this differential inequality (see Lemma 5.6). Setting $t=\theta T$ in (22) and using (32) to bound the conserved quantity gives (23) after some standard calculations.

\subsubsection{Proof of Theorem 1.5}

Proof of Theorem 1.5 Let P be optimal for (MFSP) and $\Psi$ be given by Proposition 3.1. Then if we define

$$
B_{t}:=X_{t}-\int_{0}^{t} \nabla W * \mathrm{P}_{s}\left(X_{s}\right)+\Psi_{s}\left(X_{s}\right) \mathrm{d} s
$$

the process $\left(B_{t}\right)_{t \in[0, T]}$ is a Brownian motion under P. Since the McKean Vlasov SDE admits a unique strong solution, there exists a map $\mathbb{Y}: \Omega \longrightarrow \Omega$ such that $\mathbb{Y} \circ B .:=Y$ satisfies $Y_{0}=X_{0}(\mathrm{P}-$ a.s. $)$ and

$$
\mathrm{P}-\text { a.s. } \quad Y_{t}=Y_{0}-\int_{0}^{t} \nabla W * \mathrm{P}_{s}^{\mathrm{MKv}}\left(Y_{s}\right) \mathrm{d} s+B_{t} .
$$

Define now $\delta(t)=\mathbb{E}_{\mathrm{P}}\left[\left|X_{t}-Y_{t}\right|^{2}\right]$. Using Itô's formula we get that $\delta(t)$ is differentiable with derivative

$$
\begin{aligned}
\delta^{\prime}(t)= & -2 \mathbb{E}_{\mathrm{P}}\left[\left(X_{t}-Y_{t}\right) \cdot\left(\nabla W * \mathrm{P}_{t}\left(X_{t}\right)-\nabla W * \mathrm{P}_{t}^{\mathrm{MKV}}\left(Y_{t}\right)\right)\right] \\
& +2 \mathbb{E}_{\mathrm{P}}\left[\left(X_{t}-Y_{t}\right) \cdot \Psi_{t}\left(X_{t}\right)\right]
\end{aligned}
$$

The same arguments as in Lemma 4.3 give

$$
2 \mathbb{E}_{\mathrm{P}}\left[\left(X_{t}-Y_{t}\right) \cdot\left(\nabla W * \mathrm{P}_{t}\left(X_{t}\right)-\nabla W * \mathrm{P}_{t}^{\mathrm{MKV}}\left(Y_{t}\right)\right)\right] \geq 2 \kappa \mathbb{E}_{\mathrm{P}}\left[\left|X_{t}-Y_{t}\right|^{2}\right] \geq 0
$$

Moreover, by Cauchy Schwartz:

$$
\mathbb{E}_{\mathrm{P}}\left[\left(X_{t}-Y_{t}\right) \cdot \Psi_{t}\left(X_{t}\right)\right] \leq \mathbb{E}_{\mathrm{P}}\left[\left|X_{t}-Y_{t}\right|^{2}\right]^{1 / 2} \mathbb{E}_{\mathrm{P}}\left[\left|\Psi_{t}\left(X_{t}\right)\right|^{2}\right]^{1 / 2}
$$

\footnotetext{
11 Again, the apparent mismatch between the constant in the Log Sobolev inequality from [12] and the one we use here is due to the fact that in our definition of $\tilde{\mathcal{F}}$, there is no $1 / 2$ in front of $W$.
} 
Therefore

$$
\delta^{\prime}(t) \leq 2 \delta(t)^{1 / 2} \mathbb{E}_{\mathrm{P}}\left[\left|\Psi_{t}\left(X_{t}\right)\right|^{2}\right]^{1 / 2}
$$

which gives

$$
(\sqrt{\delta})^{\prime}(t) \leq \mathbb{E}_{\mathrm{P}}\left[\left|\Psi_{t}\left(X_{t}\right)\right|^{2}\right]^{1 / 2}
$$

Integrating the differential inequality and using that $\delta(0)=0$ :

$$
\begin{array}{r}
\sqrt{\delta}(t) \leq \int_{0}^{t} \mathbb{E}_{\mathrm{P}}\left[\left|\Psi_{S}\left(X_{S}\right)\right|^{2}\right]^{1 / 2} \mathrm{~d} s \leq t^{1 / 2}\left(\int_{0}^{t} \mathbb{E}_{\mathrm{P}}\left[\left|\Psi_{S}\left(X_{s}\right)\right|^{2}\right] \mathrm{d} s\right)^{1 / 2} \\
\stackrel{(70))}{\leq} t^{1 / 2}\left(2 \frac{\exp (2 \kappa t)-1}{\exp (2 \kappa T)-1} \mathscr{C}_{T}\left(\mu^{\mathrm{in}}, \mu^{\mathrm{fin}}\right)\right)^{1 / 2} .
\end{array}
$$

The conclusion follows from (25) and the observation that $\mathcal{W}_{2}^{2}\left(\mathrm{P}_{t}, \mathrm{P}_{t}^{\mathrm{MKV}}\right) \leq \delta(t)$.

Funding Funding was provided by CMAP, Austrian Science Fund (Grant No. P30750), Agence Nationale de la Recherche (Grant No. ANR-17-CE40-0030).

Open Access This article is licensed under a Creative Commons Attribution 4.0 International License, which permits use, sharing, adaptation, distribution and reproduction in any medium or format, as long as you give appropriate credit to the original author(s) and the source, provide a link to the Creative Commons licence, and indicate if changes were made. The images or other third party material in this article are included in the article's Creative Commons licence, unless indicated otherwise in a credit line to the material. If material is not included in the article's Creative Commons licence and your intended use is not permitted by statutory regulation or exceeds the permitted use, you will need to obtain permission directly from the copyright holder. To view a copy of this licence, visit http://creativecommons.org/licenses/by/4.0/.

\section{Appendix}

We begin with the promised graphical illustration of Theorem 1.4 (Fig. 1).

Fig. 1 A qualitative illustration of the turnpike property expressed by Theorem 1.4: at first $\mathcal{F}\left(\mu_{t}\right)$ decreases exponentially fast and then it stays close to the minimum value for a long time; towards the end it abruptly increases to reach its final value

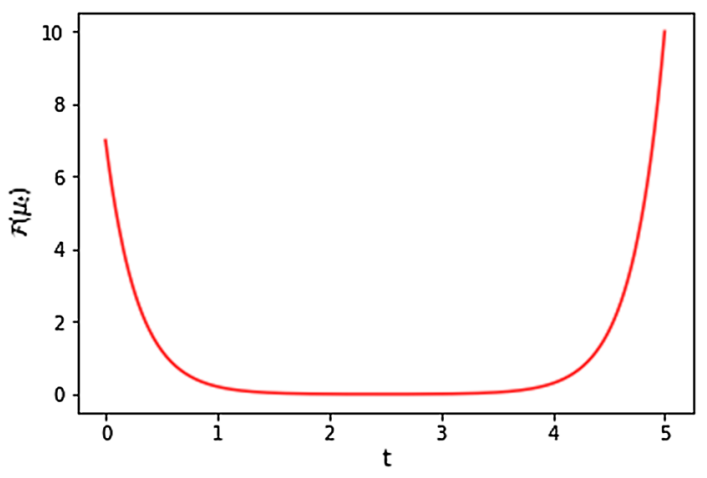


The following lemma is well known. For simple proofs see [35] or the appendix of [24].

Lemma 5.1 For $\mu \in \mathcal{P}_{2}\left(\mathbb{R}^{d}\right)$ let $\mathrm{R}^{\mu}$ be the law of the Brownian with initial law $\mu$. For $\mathrm{P} \in \mathcal{P}(\Omega)$ with $\mathrm{P} \circ\left(X_{0}\right)^{-1}=\mu$ the following are equivalent

(i) $\mathcal{H}\left(\mathrm{P} \mid \mathrm{R}^{\mu}\right)<+\infty$.

(ii) There exist a $\mathrm{P}$-a.s. defined adapted process $\left(\alpha_{t}\right)_{t \in[0, T]}$ such that

$$
\mathbb{E}_{\mathrm{P}}\left[\int_{0}^{T}\left|\alpha_{t}\right|^{2} \mathrm{~d} t\right]<+\infty
$$

and

$$
X_{t}-\int_{0}^{t} \alpha_{s} \mathrm{~d} s
$$

is a Brownian motion under $\mathrm{P}$.

Moreover, if (ii) holds, then

$$
\mathcal{H}\left(\mathrm{P} \mid \mathrm{R}^{\mu}\right)=\frac{1}{2} \mathbb{E}_{\mathrm{P}}\left[\int_{0}^{T}\left|\alpha_{t}\right|^{2} \mathrm{~d} t\right]
$$

and

$$
\mathbb{E}_{\mathrm{P}}\left[\sup _{t \in[0, T]}\left|X_{t}\right|^{2}\right]<+\infty .
$$

With the help of Lemma 5.1 we can readily prove its generalization given in Lemma 3.6 concerning the case when $R^{\mu}$ is replaced by the law of a diffusion with Lipschitz drift.

Proof of Lemma 3.6 The proof of $(i) \Rightarrow$ (ii) follows the arguments in [35]. Now assume that (ii) holds. Because of the continuity of $t \mapsto \bar{b}(t, 0)$ and (49) we get that

$$
\forall(t, x) \in[0, T] \times \mathbb{R}^{d}, \quad|\bar{b}(t, x)| \leq C^{\prime}(1+|x|)
$$

for some $C^{\prime}<+\infty$. Consider the sequence of stopping times $T_{n}=\inf \{t \geq 0$ : $\left.\left|X_{t}\right|=n\right\} \wedge T$. Using (99), (52) and some standard calculations we find that there exist $C^{\prime \prime}<+\infty$ such $\mathrm{P}$ almost surely

$$
\begin{aligned}
\forall n & \in \mathbb{N}, t \in[0, T]: \sup _{r \leq t}\left|X_{r \wedge T_{n}}\right|^{2} \\
& \leq C^{\prime \prime}\left(\left|X_{0}\right|^{2}+1+\int_{0}^{t} \sup _{r \leq s}\left|X_{r \wedge T_{n}}\right|^{2} \mathrm{~d} s+\int_{0}^{t}\left|\bar{\alpha}_{s}\right|^{2} \mathrm{~d} s+\sup _{r \leq t}\left|B_{r \wedge T_{n}}\right|^{2}\right)
\end{aligned}
$$

where $B$ is a Brownian motion. Taking expectation, using (8), using Grönwall's Lemma, and eventually letting $n \rightarrow+\infty$, one obtains

$$
\sup _{t \in[0, T]} \mathbb{E}_{\mathrm{P}}\left[\sup _{t \in[0, T]}\left|X_{t}\right|^{2}\right]<+\infty .
$$


But then, thanks to (99) and (51) we also obtain that $\mathbb{E}_{\mathrm{P}}\left[\int_{0}^{T}\left|\bar{b}\left(s, X_{s}\right)+\bar{\alpha}_{s}\right|^{2} \mathrm{~d} s\right]<+\infty$. Lemma 5.1 yields then (as usual $\mathrm{R}^{\mu}$ is Wiener measure started like $\mu$ )

$$
\mathcal{H}\left(\mathrm{P} \mid \mathrm{R}^{\mu}\right)<+\infty, \quad \text { and } \mathcal{H}\left(\mathrm{P} \mid \mathrm{R}^{\mu}\right)=\frac{1}{2} \mathbb{E}_{\mathrm{P}}\left[\int_{0}^{T}\left|\bar{b}\left(t, X_{t}\right)+\bar{\alpha}_{t}\right|^{2} \mathrm{~d} t\right]
$$

Under the current hypotheses on $b, \mathrm{R}^{\mu}$ and $\overline{\mathrm{R}}$ are mutually absolutely continuous and

$$
\frac{\mathrm{dR}^{\mu}}{\mathrm{d} \overline{\mathrm{R}}}=\exp \left(-\int_{0}^{T} \bar{b}\left(t, X_{t}\right) \cdot \mathrm{d} X_{t}+\frac{1}{2} \int_{0}^{T}\left|\bar{b}\left(t, X_{t}\right)\right|^{2} \mathrm{~d} t\right) .
$$

Therefore, using some standard calculations and (ii) we get

$$
\mathbb{E}_{\mathrm{P}}\left[\log \frac{\mathrm{dR}^{\mu}}{\mathrm{d} \overline{\mathrm{R}}}\right]=-\mathbb{E}_{\mathrm{P}}\left[\int_{0}^{T}\left(\bar{\alpha}_{t}+\frac{1}{2} \bar{b}\left(t, X_{t}\right)\right) \cdot \bar{b}\left(t, X_{t}\right) \mathrm{d} t\right]<+\infty .
$$

Since $\mathrm{R}^{\mu}$ and $\overline{\mathrm{R}}$ are mutually absolutely continuous and $\mathcal{H}\left(\mathrm{P} \mid \mathrm{R}^{\mu}\right)<+\infty$ we get that $\mathrm{P} \ll \overline{\mathrm{R}}$ and

$$
\mathcal{H}(\mathrm{P} \mid \overline{\mathrm{R}})=\mathbb{E}_{\mathrm{P}}\left[\log \frac{\mathrm{dP}}{\mathrm{dR}^{\mu}}+\log \frac{\mathrm{dR}^{\mu}}{\mathrm{d} \overline{\mathrm{R}}}\right]
$$

Thus, $\mathcal{H}(\mathrm{P} \mid \overline{\mathrm{R}})<+\infty$ if both $\mathcal{H}\left(\mathrm{P} \mid \mathrm{R}^{\mu}\right)$ and $\mathbb{E}_{\mathrm{P}}\left[\log \frac{\mathrm{dR}^{\mu}}{\mathrm{d} \overline{\mathrm{R}}}\right]$ are finite. But this is indeed the case, thanks to (102), (101). The proof that $(i i) \Rightarrow(i)$ is now complete. The desired form of the relative entropy follows by plugging in (101) and (102) into (103). Finally (54) follows from (100) and (99), and the last statement from (54).

Lemma 5.2 Let $\mu^{\text {in }} \in \mathcal{P}\left(\mathbb{R}^{d}\right)$ and $\mathrm{P} \in \mathcal{P}_{1}(\Omega)$ with $\mathrm{P}_{0}=\mu^{\text {in }}$. Then $\mathcal{H}(\mathrm{P} \mid \Gamma(\mathrm{P}))<+\infty$ if and only if $\mathcal{H}\left(\mathrm{P} \mid \mathrm{R}^{\mu^{\text {in }}}\right)<+\infty$.

Proof Define $\bar{b}(t, z)=-\nabla W * \mathrm{P}_{t}(z)$. Lemma 3.5 ensures that $\bar{b}$ is of class $\mathcal{C}^{0,1}$ and that (49) holds. Assume that $\mathcal{H}(\mathrm{P} \mid \Gamma(\mathrm{P}))<+\infty$ and let $\left(\bar{\alpha}_{t}\right)_{t \in[0, T]}$ be given by the implication $(i) \Rightarrow(i i)$ of Lemma 3.6. If we define $\left(\alpha_{t}\right)_{t \in[0, T]}$ by

$$
\mathrm{P}-\text { a.s. } \quad \alpha_{t}=\bar{b}\left(t, X_{t}\right)+\bar{\alpha}_{t}, \quad \forall t \in[0, T],
$$

then (54) together with (51) entitle us to use the implication ( $i i) \Rightarrow(i)$ of Lemma 5.1 to obtain the desired result. The converse implication is done inverting the roles of Lemmas 3.6 and 5.1.

Lemma 5.3 Assume (H1), (H2) $\mathrm{P}, \mathrm{Q} \in \mathcal{P}_{1}(\Omega)$ be such that $\mathcal{H}(\mathrm{P} \mid \Gamma(\mathrm{P})), \mathcal{H}(\mathrm{Q} \mid \Gamma(\mathrm{P}))<$ $+\infty$ and $\left(\mathrm{Q}_{t}\right)_{t \in[0, T]}=\left(\mathrm{P}_{t}\right)_{t \in[0, T]}$. If $\left(\theta^{n}\right)_{n \in \mathbb{N}} \subseteq \mathcal{C}_{c}^{\infty}\left([0, T] \times \mathbb{R}^{d}\right)$ is such that $\nabla \theta^{n}$ converges to $-\nabla W * \mathrm{P}_{t}(z)$ in $\mathrm{H}_{-1}\left(\left(\mathrm{P}_{t}\right)_{t \in[0, T]}\right)$, i.e.

$$
\lim _{n \rightarrow+\infty} \int_{[0, T] \times \mathbb{R}^{d}}\left|\nabla \theta_{t}^{n}(z)+\nabla W * \mathrm{P}_{t}(z)\right|^{2} \mathrm{P}_{t}(\mathrm{~d} z) \mathrm{d} t=0
$$


then

$$
\lim _{n \rightarrow+\infty} \mathcal{H}\left(\mathrm{Q} \mid \mathrm{R}^{n}\right)=\mathcal{H}(\mathrm{Q} \mid \Gamma(\mathrm{P}))
$$

where $\mathrm{R}^{n}$ is the law of

$$
\mathrm{d} Y_{t}=\nabla \theta_{t}^{n}\left(Y_{t}\right) \mathrm{d} t+\mathrm{d} B_{t}, \quad Y_{0} \sim \mu^{\text {in }} \in \mathcal{P}_{2}\left(\mathbb{R}^{d}\right) .
$$

Proof By Lemma 5.2 we have $\mathcal{H}\left(\mathrm{Q} \mid \mathrm{R}^{\mu^{\text {in }}}\right)<+\infty$, where $R^{\mu^{\text {in }}}$ is the law of the Brownian motion with initial law $\mu^{\text {in }}$. Using implication $(i) \Rightarrow$ (ii) from Lemma 5.1 and then $(i i) \Rightarrow(i)$ together with (53) from Lemma 3.6 for the choice $\bar{b}=\nabla \theta^{n}$, we get for all $n \in \mathbb{N}$ :

$$
\mathcal{H}\left(\mathrm{Q} \mid \mathrm{R}^{n}\right)=\frac{1}{2} \mathbb{E}_{\mathrm{Q}}\left[\int_{0}^{T}\left|\alpha_{t}-\nabla \theta_{t}^{n}\left(X_{t}\right)\right|^{2} \mathrm{~d} t\right],
$$

where $\alpha_{t}$ is the dirft of Q (see Lemma 5.1). Moreover, using $\mathcal{H}(\mathrm{Q} \mid \Gamma(\mathrm{P}))<+\infty$ and Lemma 3.6, we also get that

$$
\mathcal{H}(\mathrm{Q} \mid \Gamma(\mathrm{P}))=\frac{1}{2} \mathbb{E}_{\mathrm{Q}}\left[\int_{0}^{T}\left|\alpha_{t}+\nabla W * \mathrm{P}_{t}\left(X_{t}\right)\right|^{2} \mathrm{~d} t\right]
$$

Using $\left(\mathrm{Q}_{t}\right)_{t \in[0, T]}=\left(\mathrm{P}_{t}\right)_{t \in[0, T]}$ and (104) we get

$$
\lim _{n \rightarrow+\infty} \mathbb{E}_{\mathrm{Q}}\left[\int_{0}^{T}\left|\nabla \theta_{t}^{n}\left(X_{t}\right)\right|^{2} \mathrm{~d} t\right]=\mathbb{E}_{\mathrm{Q}}\left[\int_{0}^{T}\left|\nabla W * \mathrm{P}_{t}\left(X_{t}\right)\right|^{2} \mathrm{~d} t\right]
$$

On the other hand, let $\bar{\alpha}_{t}\left(X_{t}\right)$ be a measurable version of $\mathbb{E}_{\mathrm{Q}}\left[\alpha_{t} \mid X_{t}\right]$, the existence of which is guaranteed e.g. by [30, Proposition 4.4]. Using conditional Jensen and $\mathbb{E}_{\mathrm{Q}}\left[\int_{0}^{T}\left|\alpha_{t}\right|^{2} \mathrm{~d} t\right]<+\infty$, it is easy to verify that $\bar{\alpha} \in \mathrm{H}_{-1}\left(\left(\mathrm{P}_{t}\right)_{t \in[0, T]}\right)$. Moreover

$$
\mathbb{E}_{\mathrm{Q}}\left[\int_{0}^{T} \alpha_{t} \cdot \nabla \theta_{t}^{n}\left(X_{t}\right) \mathrm{d} t\right]=\int_{[0, T] \times \mathbb{R}^{d}} \bar{\alpha}_{t}(z) \cdot \nabla \theta_{t}^{n}(z) \mathrm{P}_{t}(\mathrm{~d} z) \mathrm{d} t
$$

Since $\bar{\alpha} \in \mathrm{H}_{-1}\left(\left(\mathrm{P}_{t}\right)_{t \in[0, T]}\right)$ we get from (104), $\left(\mathrm{Q}_{t}\right)_{t \in[0, T]}=\left(\mathrm{P}_{t}\right)_{t \in[0, T]}$ and the basic properties of conditional expectation

$$
\lim _{n \rightarrow+\infty} \int_{[0, T] \times \mathbb{R}^{d}} \bar{\alpha}_{t}(z) \cdot \nabla \theta_{t}^{n}(z) \mathrm{P}_{t}(\mathrm{~d} z) \mathrm{d} t=\mathbb{E}_{\mathrm{Q}}\left[\int_{0}^{T} \alpha_{t} \cdot \nabla W * \mathrm{P}_{t}\left(X_{t}\right) \mathrm{d} t\right]
$$

Gathering (106), (108), (105), (107) the conclusion follows. 
Lemma 5.4 Assume $\mathrm{P} \ll \mathrm{R}^{\mu}$ and that $\left(h_{t}\right)_{t \in[0, T]}$ is an adapted process satisfying the Lipschitz condition in (63). Then the shift $\tau_{\varepsilon}$ defined at (64) admits an almost sure inverse, i.e. there exists an adapted process $\left(Y_{t}^{\varepsilon}\right)_{t \in[0, T]}$ such that

$$
\mathrm{P}-\text { a.s. } \tau_{t}^{\varepsilon}\left(Y^{\varepsilon}(\omega)\right)=Y_{t}^{\varepsilon}\left(\tau^{\varepsilon}(\omega)\right)=\omega_{t} \quad \forall t \in[0, T] .
$$

Proof Let $\mathrm{R}^{\mu}$ be the law of the Brownian motion started at $\mu$. The fact that (109) holds $\mathrm{R}^{\mu}$ almost surely under the Lipschitz condition (63) is a classical result, see e.g. [52, pp. 209-210]. In this case the a.s. inverse is nothing but the strong solution of the SDE

$$
\mathrm{d} Y_{t}^{\varepsilon}=-\varepsilon h_{t}\left(Y_{t}^{\varepsilon}\right) \mathrm{d} t+\mathrm{d} B_{t}
$$

We conclude by $\mathrm{P} \ll \mathrm{R}^{\mu}$.

The next lemma follows from [17, Lemma 4.1]:

Lemma 5.5 Let $c:[0, T] \rightarrow \mathbb{R}$ be twice differentiable on $(0, T)$ with $c(0)=0$ and let $\kappa \in \mathbb{R}$. If $\frac{\mathrm{d}^{2}}{\mathrm{~d} t^{2}} c(t) \geq 2 \kappa \frac{\mathrm{d}}{\mathrm{d} t} c(t)$ for all $t \in(0, T)$, then

$$
\forall t \in[0, T], \quad c(t) \leq \frac{\exp (2 \kappa t)-1}{\exp (2 \kappa T)-1} c(T)
$$

The following lemma is also useful for the quantitative estimates:

Lemma 5.6 Let $\varphi:[0, T] \rightarrow \mathbb{R}$ be twice differentiable on $(0, T)$ and assume that $\varphi(t)$ satisfies the differential inequality

$$
\frac{\mathrm{d}^{2}}{\mathrm{~d} t^{2}} \varphi_{t} \geq \lambda^{2} \varphi_{t}-\lambda \mathscr{E}
$$

where $\mathscr{E}$ is a constant. Then we have for all $0 \leq t \leq T$,

$$
\varphi_{t} \leq\left(\varphi_{0}-\frac{\mathscr{E}}{\lambda}\right) \frac{\sinh (\lambda(T-t))}{\sinh (\lambda T)}+\left(\varphi_{T}-\frac{\mathscr{E}}{\lambda}\right) \frac{\sinh (\lambda t)}{\sinh (\lambda T)}+\frac{\mathscr{E}}{\lambda}
$$

Proof Consider the function $\gamma_{t}=\varphi_{t}-\psi_{t}$, where $\psi_{t}$ is the right hand side of (111). It is easily verified that $\frac{\mathrm{d}^{2}}{\mathrm{~d} t^{2}} \psi_{t}=\lambda^{2} \psi_{t}-\lambda \mathscr{E}$ and $\psi_{0}=\varphi_{0}, \psi_{T}=\varphi_{T}$. Consequently, we have that $\frac{\mathrm{d}^{2}}{\mathrm{~d} t^{2}} \gamma_{t} \geq \lambda^{2} \gamma_{t}$ for $t \in[0, T]$ and $\gamma_{0}=\gamma_{T}=0$. Assume $\gamma_{t_{1}}>0$ for some $t_{1}$. Since $\gamma_{0}=0$, there must exist $t_{0} \leq t_{1}$ such that $\gamma_{t_{0}}>0$ and $\frac{\mathrm{d}}{\mathrm{d} t} \gamma_{t_{0}}>0$. But this contradicts Lemma 5.7 below.

Lemma 5.7 Assume that $\lambda \geq 0$. Let $0 \leq t_{0}<T$ and $\gamma:[0, T] \rightarrow \mathbb{R}$ be a function satisfying

$$
\left\{\begin{array}{l}
\frac{\mathrm{d}^{2}}{\mathrm{~d} t^{2}} \gamma_{t} \geq \lambda \gamma_{t}, t \in\left[t_{0}, T\right] \\
\gamma_{t_{0}}>0, \gamma_{T}=0
\end{array}\right.
$$


Then $\frac{\mathrm{d}}{\mathrm{d} t} \gamma_{t_{0}} \leq 0$.

Proof Assume ad absurdum that $\frac{\mathrm{d}}{\mathrm{d} t} \gamma_{t_{0}}>0$. Since $\gamma_{T}=0$ it must be that $t_{1}=\inf \{t \geq$ $\left.t_{0} ; \frac{\mathrm{d}}{\mathrm{d} t} \gamma_{t}=0\right\}$ belongs to $\left(t_{0}, T\right]$. By definition of $t_{1}$, and since $\frac{\mathrm{d}}{\mathrm{d} t} \gamma_{t_{0}}>0$ we have that $\gamma_{t} \geq \gamma_{t_{0}}>0$ for all $t \in\left[t_{0}, t_{1}\right]$. But then

$$
0>\frac{\mathrm{d}}{\mathrm{d} t} \gamma_{t_{1}}-\frac{\mathrm{d}}{\mathrm{d} t} \gamma_{t_{0}}=\int_{t_{0}}^{t_{1}} \frac{\mathrm{d}^{2}}{\mathrm{~d} t^{2}} \gamma_{t} \mathrm{~d} t \geq \lambda \int_{t_{0}}^{t_{1}} \gamma_{t} \mathrm{~d} t \geq 0,
$$

which is absurd.

\section{References}

1. Ambrosio, L., Gigli, N., Savaré, G.: Gradient Flows: In Metric Spaces and in the Space of Probability Measures. Springer, Berlin (2008)

2. Benamou, J.-D., Carlier, G. Di Marino, S., Nenna, L.: An entropy minimization approach to secondorder variational mean-field games. arXiv preprint arXiv:1807.09078 (2018)

3. Bolley, F., Gentil, I., Guillin, A.: Uniform convergence to equilibrium for granular media. Arch. Ration. Mech. Anal. 208(2), 429-445 (2013)

4. Budhiraja, A., Dupuis, P., Fischer, M.: Large deviation properties of weakly interacting processes via weak convergence methods. Ann. Probab. 40(1), 74-102 (2012)

5. Cardaliaguet, P.: Long time average of first order mean field games and weak kam theory. Dyn. Games Appl. 3(4), 473-488 (2013)

6. Cardaliaguet, P.: Notes on mean field games. Technical report. https://www.ceremade.dauphine.fr/ cardaliaguet/ (2013)

7. Cardaliaguet, P., Lasry, J.-M., Lions, P.-L., Porretta, A.: Long time average of mean field games. NHM 7(2), 279-301 (2012)

8. Cardaliaguet, P., Lasry, J.-M., Lions, P.-L., Porretta, A.: Long time average of mean field games with a nonlocal coupling. SIAM J. Control Optim. 51(5), 3558-3591 (2013)

9. Cardaliaguet, P., Porretta, A.: Long time behavior of the master equation in mean field game theory. Anal. PDE 12(6), 1397-1453 (2019)

10. Carmona, R., Delarue, F.: Probabilistic analysis of mean-field games. SIAM J. Control Optim. 51(4), 2705-2734 (2013)

11. Carmona, R., Delarue, F.: Forward-backward stochastic differential equations and controlled McKeanVlasov dynamics. Ann. Probab. 43(5), 2647-2700 (2015)

12. Carrillo, J., McCann, R., Villani, C.: Kinetic equilibration rates for granular media and related equations: entropy dissipation and mass transportation estimates. Rev. Mat. Iberoam. 19(3), 971-1018 (2003)

13. Cattiaux, P., Guillin, A., Malrieu, F.: Probabilistic approach for granular media equations in the nonuniformly convex case. Probab. Theory Relat. Fields 140(1-2), 19-40 (2008)

14. Cattiaux, P., Léonard, C.: Large deviations and Nelson processes. Forum Mathematicum, vol. 7, pp. 95-116. Walter de Gruyter, Berlin (1995)

15. Chen, Y., Georgiou, T., Pavon, M.: On the relation between optimal transport and Schrödinger bridges: a stochastic control viewpoint. J. Optim. Theory Appl. 169(2), 671-691 (2016)

16. Chen, Y., Georgiou, T., Pavon, M.: Steering the distribution of agents in mean-field games system. J. Optim. Theory Appl. 179(1), 332-357 (2018)

17. Conforti, G.: A second order equation for Schrödinger bridges with applications to the hot gas experiment and entropic transportation cost. Probab. Theory Relat. Fields 174(1-2), 1-47 (2019)

18. Cruzeiro, A.B., Lassalle, R.: Weak calculus of variations for functionals of laws of semi-martingales. arXiv preprint arXiv:1501.05134 (2015)

19. Cuturi, M.: Sinkhorn distances: lightspeed computation of optimal transport. In: Advances in Neural Information Processing Systems, pp. 2292-2300 (2013)

20. Dawson, D., Gärtner, J.: Large deviations from the Mckean-Vlasov limit for weakly interacting diffusions. Stochastics 20(4), 247-308 (1987) 
21. Dembo, A., Zeitouni, O.: Large Deviations Techniques and Applications, vol. 38. Springer, Berlin (2009)

22. Doob, J.: Conditional Brownian motion and the boundary limits of harmonic functions. Bull. Soc. Math. France 85, 431-458 (1957)

23. Émery, M.: En cherchant une caractérisation variationnelle des martingales. In: Séminaire de Probabilités XXII, pp. 147-154. Springer (1988)

24. Fischer, M.: On the form of the large deviation rate function for the empirical measures of weakly interacting systems. Bernoulli 20(4), 1765-1801 (2014)

25. Föllmer, H.: Time reversal on Wiener space. In: Stochastic Processes-Mathematics and Physics, pp. 119-129. Springer (1986)

26. Föllmer, H.: Random fields and diffusion processes. In: École d'Été de Probabilités de Saint-Flour XV-XVII, 1985-87, pp. 101-203. Springer (1988)

27. Gentil, I., Léonard, C., Ripani, L.: About the analogy between optimal transport and minimal entropy. Ann. Fac. Sci. Toulouse Sér. 6 26(3), 569-700 (2017)

28. Gentil, I., Léonard, C., Ripani, L.: Dynamical aspects of generalized Schrödinger problem via otto calculus - a heuristic point of view. Rev. Mat. Iberoam. (2018, to appear)

29. Gigli, N.: Second Order Analysis on (P2 (M), W2). American Mathematical Society, Providence (2012)

30. Gyöngy, I.: Mimicking the one-dimensional marginal distributions of processes having an Itô differential. Probab. Theory Relat. Fields 71(4), 501-516 (1986)

31. Jordan, R., Kinderlehrer, D., Otto, F.: The variational formulation of the Fokker-Planck equation. SIAM J. Math. Anal. 29(1), 1-17 (1998)

32. Karatzas, I., Schachermayer, W., Tschiderer, B.: Pathwise Otto calculus. arXiv preprint arXiv:1811.08686 (2018)

33. Lasry, J.-M., Lions, P.-L.: Mean field games. Jpn. J. Math. 2(1), 229-260 (2007)

34. Léger, F., Li, W.: Hopf-Cole transformation via generalized Schrödinger bridge problem. arXiv preprint arXiv:1901.09051 (2019)

35. Léonard, C.: Girsanov theory under a finite entropy condition. In: Donati-Martin, C., Lejay, A., Rouault, A. (eds.) Séminaire de Probabilités XLIV, Lecture Notes in Mathematics, vol. 2046, pp. 429-465. Springer (2012)

36. Léonard, C.: A survey of the Schrödinger problem and some of its connections with optimal transport. Discrete Contin. Dyn. Syst. 34(4), 1533-1574 (2014)

37. Léonard, C., Rœlly, S., Zambrini, J.-C.: Reciprocal processes. a measure-theoretical point of view. Probab. Surv. 11, 237-269 (2014)

38. McCann, R.: A convexity principle for interacting gases. Adv. Math. 128(1), 153-179 (1997)

39. McKenzie, L.: Turnpike theorems for a generalized Leontief model. Econometrica (pre-1986) 31(1, 2), 165 (1963)

40. Mikami, T.: Monge's problem with a quadratic cost by the zero-noise limit of h-path processes. Probab. Theory Relat. Fields 129(2), 245-260 (2004)

41. Otto, F.: The geometry of dissipative evolution equations: the porous medium equation. Commun. Partial Differ. Equ. 26(1-2), 101-174 (2001)

42. Otto, F., Villani, C.: Generalization of an inequality by Talagrand and links with the logarithmic Sobolev inequality. J. Funct. Anal. 173(2), 361-400 (2000)

43. Pal, S., Wong, T.-K.L.: Multiplicative Schrödinger problem and the Dirichlet transport. arXiv preprint arXiv:1807.05649 (2018)

44. Ripani, L.: Convexity and regularity properties for entropic interpolations. arXiv preprint arXiv:1711.08230 (2017)

45. Rogers, C., Williams, D.: Diffusions, Markov Processes and Martingales: Volume 2, Itô Calculus, vol. 2. Cambridge University Press, Cambridge (2000)

46. Schrödinger, E.: Über die Umkehrung der Naturgesetze. Sitzungsberichte Preuss. Akad. Wiss. Berlin. Phys. Math. 144, 144-153 (1931)

47. Schrödinger, E.: La théorie relativiste de l'électron et l' interprétation de la mécanique quantique. Ann. Inst Henri Poincaré 2, 269-310 (1932)

48. Talagrand, M.: Transportation cost for Gaussian and other product measures. Geom. Funct. Anal. GAFA 6(3), 587-600 (1996)

49. Tanaka, H.: Limit theorems for certain diffusion processes with interaction. In: North-Holland Mathematical Library, vol. 32, pp. 469-488. Elsevier (1984) 
50. Trélat, E., Zuazua, E.: The turnpike property in finite-dimensional nonlinear optimal control. J. Differ. Equ. 258(1), 81-114 (2015)

51. Tugaut, J.: Phase transitions of Mckean-Vlasov processes in double-wells landscape. Stochastics $\mathbf{8 6}(2)$, 257-284 (2014)

52. Üstünel, A.S., Zakai, M.: Sufficient conditions for the invertibility of adapted perturbations of identity on the Wiener space. Probab. Theory Relat. Fields 139(1-2), 207-234 (2007)

53. Villani, C.: Optimal transport: old and new, vol. 338. Springer, Berlin (2008)

54. von Renesse, M.: An optimal transport view of Schrödinger's equation. Can. Math. Bull. 55(4), 858869 (2012)

55. von Renesse, M., Sturm, K.-T.: Transport inequalities, gradient estimates, entropy and Ricci curvature. Commun. Pure Appl. Math. 58(7), 923-940 (2005)

56. Wang, R., Wang, X., Liming, W.: Sanov's theorem in the Wasserstein distance: a necessary and sufficient condition. Stat. Probab. Lett. 80(5-6), 505-512 (2010)

57. Zambrini, J.-C.: Variational processes and stochastic versions of mechanics. J. Math. Phys. 27(9), 2307-2330 (1986)

Publisher's Note Springer Nature remains neutral with regard to jurisdictional claims in published maps and institutional affiliations. 